\title{
Monitoring the Antimicrobial Resistance Dynamics of Salmonella enterica in Healthy Dairy Cattle Populations at the Individual Farm Level Using Whole-Genome Sequencing
}

1 Laura M. Carroll ${ }^{* \#}$, Ariel J. Buehler ${ }^{1}$, Ahmed Gaballa ${ }^{1}$, Julie D. Siler ${ }^{2}$, Kevin J. Cummings ${ }^{2}$,

2 Rachel A. Cheng ${ }^{1}$, Martin Wiedmann ${ }^{1}$

$3 \quad{ }^{1}$ Department of Food Science, Cornell University, Ithaca, New York, USA

$4 \quad{ }^{2}$ Department of Population Medicine and Diagnostic Sciences, Cornell University, Ithaca, New York, 5 USA

* Correspondence:

7 Laura M. Carroll

8 1mc297@cornell.edu

9 " Present address: Laura M. Carroll, Structural and Computational Biology Unit, EMBL,

10 Heidelberg, Germany.

Keywords: Salmonella, antimicrobial resistance, serotyping, dairy cattle, whole-genome sequencing, evolution, livestock

Abstract

Livestock represent a possible reservoir for facilitating the transmission of the zoonotic foodborne pathogen Salmonella enterica to humans; there is also concern that strains can acquire resistance to antimicrobials in the farm environment. Here, we use whole-genome sequencing (WGS) to characterize Salmonella strains $(n=128)$ isolated from healthy dairy cattle and their associated environments on $13 \mathrm{New}$ York State farms to assess the diversity and microevolution of this important pathogen at the level of the individual herd. Additionally, the accuracy and concordance of multiple in silico tools are assessed, including: (i) two in silico serotyping tools, (ii) combinations of five antimicrobial resistance (AMR) determinant detection tools and one to five AMR determinant databases, and (iii) one antimicrobial minimum inhibitory concentration (MIC) prediction tool. For the isolates sequenced here, in silico serotyping methods outperformed traditional serotyping and resolved all un-typable and/or ambiguous serotype assignments. Serotypes assigned in silico showed greater congruency with the Salmonella whole-genome phylogeny than traditional serotype assignments, and in silico methods showed high concordance ( $99 \%$ agreement). In silico AMR determinant detection methods additionally showed a high degree of concordance, regardless of the pipeline or database used ( $\geq 98 \%$ agreement between susceptible/resistant assignments for all pipeline/database combinations). For AMR detection methods that relied exclusively on nucleotide BLAST, accuracy could be maximized by using a range of minimum nucleotide identity and coverage thresholds, with thresholds of $75 \%$ nucleotide identity and $50-60 \%$ coverage adequate for most pipeline/database combinations. In silico characterization of the microevolution and AMR dynamics of each of six serotype groups ( $S$. Anatum, Cerro, Kentucky, Meleagridis, Newport, Typhimurium/Typhimurium variant Copenhagen) revealed that some lineages were strongly associated with individual farms, while others were distributed across multiple farms. Numerous 
AMR determinant acquisition and loss events were identified, including the recent acquisition of

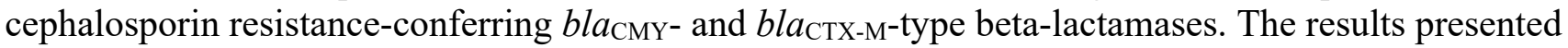
here provide high-resolution insight into the temporal dynamics of AMR Salmonella at the scale of the individual farm and highlight both the strengths and limitations of WGS in tracking zoonotic pathogens and their associated AMR determinants at the livestock-human interface.

\section{Introduction}

The foodborne pathogen Salmonella enterica is estimated to be responsible for 1.2 million

Cattle are a potential reservoir from which humans can acquire salmonellosis, and infected animals can shed Salmonella at irregular intervals for varying periods of time, regardless of whether they express clinical signs of bovine salmonellosis or not (Cummings et al., 2010b; Davidson et al., 2018; Holschbach and Peek, 2018). The bovine reservoir boasts its own repertoire of serotypes that can infect humans, with bovine-associated Salmonella serotype Dublin, known for its rare but frequently invasive infections in humans, being arguably the most noteworthy (Taylor et al., 1982; Uzzau et al., 2000; Rodriguez-Rivera et al., 2014; Harvey et al., 2017; Mohammed et al., 2017). However, a range of Salmonella serotypes can persist and thrive in cattle, potentially infecting humans via either direct contact with infected animals or through food (Gutema et al., 2019). In a previous survey of 46 dairy cattle herds in New York State, Salmonella strains isolated from subclinically infected dairy cattle and associated farm environments spanned 26 serotypes, the most common being Cerro, Kentucky, Typhimurium, Newport, and Anatum (Rodriguez-Rivera et al., 2014). Additionally, antimicrobial-resistant (AMR) isolates were observed on several farms, on numerous occasions, suggesting subclinically infected dairy cattle as a potential source of AMR Salmonella (Rodriguez-Rivera et al., 2014).

Numerous studies have employed whole-genome sequencing (WGS) to characterize Salmonella from bovine sources (Mather et al., 2013; Agren et al., 2016; Carroll et al., 2017b; Delgado-Suarez et al., 2018; Liao et al., 2019); however, little is known regarding the evolution and AMR acquisition and loss dynamics of Salmonella at the single herd/farm level. Furthermore, the bulk of bovine-associated Salmonella WGS efforts have focused on clinical veterinary samples and/or epidemic lineages (e.g., S. Typhimurium DT104). In this study, 128 non-typhoidal Salmonella enterica strains isolated from repeated sampling on 13 New York State dairy cattle farms between 2007 and 2009 were characterized using WGS. All strains were isolated from apparently healthy, subclinically infected bovine hosts, as well as the associated farm environment (Rodriguez-Rivera et al., 2014). Using WGS, we characterized the microevolution of these persistent lineages within each herd, as well as the temporal acquisition and loss of AMR determinants among them. In addition to offering insight into the genomics of Salmonella isolated from healthy bovine populations at the individual herd/farm level, we evaluate the accuracy and concordance of multiple in silico serotyping and AMR prediction tools. Finally, we provide an in-depth, critical analysis of the strengths and 
limitations of the methods used here, and we offer guidance to researchers who wish to employ WGS

82 for herd-level pathogen monitoring.

\section{Materials and Methods}

\subsection{Isolate Selection}

85

86

87

88

89

90

91

92

93

Salmonella enterica isolates $(n=128)$ obtained from one of 13 dairy farms in New York State were selected to undergo WGS for this study (Supplementary Table S1). All strains were isolated from farms that had undergone surveillance for Salmonella for a period of at least 12 months as described previously (Cummings et al., 2010a; Rodriguez-Rivera et al., 2014). Strains were isolated from repeated sampling on each farm between October 2007 and August 2009, from either (i) fecal samples from healthy, subclinically infected dairy cows (referred to hereafter as "bovine" isolates), or (ii) farm environmental swabs (referred to hereafter as "farm environmental" isolates) (Cummings et al., 2010a). All isolates underwent serotyping, phenotypic antimicrobial susceptibility testing, and pulsed-field gel electrophoresis (PFGE) as described previously (Rodriguez-Rivera et al., 2014).

\subsection{Whole-Genome Sequencing and Data Pre-Processing}

Genomic DNA extraction and sequencing library preparation were performed as described previously (Carroll et al., 2017b), and the genomes of all 128 Salmonella isolates were sequenced using an Illumina HiSeq platform and 2 x 250 bp paired-end reads. Illumina sequencing adapters and low-quality bases were trimmed using Trimmomatic version 0.33 (using default parameters for Nextera paired-end reads) (Bolger et al., 2014), and FastQC version 0.11.9 (Andrews, 2019) was used to confirm adapter removal and assess read quality. SPAdes version 3.8.0 (Bankevich et al., 2012) was used to assemble genomes de novo (using the "careful" option and $k$-mer sizes of 21,33 , 55, 77, 99, and 127), and QUAST version 4.5 (Gurevich et al., 2013) and the "lineage_wf" workflow implemented in CheckM version 1.1.3 (Parks et al., 2015) were used to assess the quality of the resulting assemblies. MultiQC version 1.8 (Ewels et al., 2016) was used to aggregate genome quality metrics. Genome quality statistics are available for all isolates (Supplementary Table S1).

\subsection{In Silico Serotyping}

In addition to undergoing traditional serotyping in a laboratory setting (i.e., serological detection of expressed $\mathrm{O}$ and $\mathrm{H}$ antigens using the White-Kauffmann-Le Minor scheme) as described previously (Rodriguez-Rivera et al., 2014), all 128 assembled Salmonella genomes (see section "Whole-Genome Sequencing and Data Pre-Processing" above) underwent in silico serotyping using the command line implementations of (i) the Salmonella In Silico Typing Resource (SISTR) version 1.0.2 (Yoshida et al., 2016) and (ii) SeqSero2 version 1.1.1 (Zhang et al., 2019) (using SeqSero2's $k$ mer based workflow). Serotypes assigned using all three methods are available for all 128 isolates (Supplementary Table S1). In cases where a discrepancy existed between the traditional serotype designation and one or more of the in silico methods, the serotype assigned using two out of the three methods was selected as the final serotype to be reported (e.g., when assigning strain names to isolates in the manuscript, for phylogeny annotation). To confirm that all serotype assignments were reasonable, a phylogeny was constructed using core single nucleotide polymorphisms (SNPs) detected in all Salmonella genomes in this study (see section "Reference-Free SNP Identification and Phylogeny Construction" below). 


\subsection{In Silico Antimicrobial Resistance Determinant Detection}

Antimicrobial resistance (AMR) determinants were detected in each of the 128 Salmonella genomes using five separate approaches: (i) ABRicate (https://github.com/tseemann/abricate) version 0.8 (Seemann, 2018), (ii) AMRFinderPlus version 3.2.3 (Feldgarden et al., 2019), (iii) ARIBA version 2.14.1 (Hunt et al., 2017), (iv) BTyper version 2.3.3 (Carroll et al., 2017a), and (v) SRST2 version 0.2.0 (Inouye et al., 2014). Assembled genomes were used as input for the ABRicate and BTyper approaches, while trimmed Illumina reads were used as input for the SRST2 and ARIBA approaches. Prokka version 1.12 (Seemann, 2014) was used to annotate each assembled genome, and the resulting GFF (.gff) and FASTA (.faa and .ffn) files were used as input for the AMRFinderPlus approach. For the ABRicate approach, the following AMR gene databases were tested (each accessed June 11, 2018 via ABRicate's abricate-get_db command): (i) the Antibiotic Resistance GeneANNOTation database (ARG-ANNOT) (Gupta et al., 2014), (ii) the Comprehensive Antibiotic Resistance Database (CARD) (Jia et al., 2017), (iii) the National Center for Biotechnology Information's (NCBI's) Bacterial Antimicrobial Resistance Reference Gene Database (NCBI) (Feldgarden et al., 2019), and (iv) the ResFinder database (ResFinder) (Zankari et al., 2012). For each genome and database combination, minimum AMR gene identity and coverage thresholds ranging from $50-100 \%$ (5\% increments) and $0-100 \%$ (10\% increments) were tested, respectively. For the BTyper approach, the (i) ARG-ANNOT v3 and (ii) MEGARes version 1.0.1 (Lakin et al., 2017) databases available with BTyper version 2.3.3 were used, with the minimum AMR gene identity and coverage thresholds varied in a manner identical to the ABRicate approach. For the SRST2 approach, the (i) ARG-ANNOT and (ii) ResFinder databases available with SRST2 version 0.2.0 were tested, using default thresholds. For the ARIBA approach, the following databases were tested (each accessed June 13, 2019 using ARIBA's getref command): (i) the version of ARG-ANNOT available with SRST2, (ii) CARD, (iii) MEGARes, (iv) NCBI, and (v) ResFinder, with all default thresholds used. For the AMRFinder approach, the latest version of the AMRFinder database was used (accessed December 6, 2019), along with the organism-specific database for Salmonella.

\subsection{In Silico Prediction of Antimicrobial Minimum Inhibitory Concentration Values}

The PATRIC3 antimicrobial minimum inhibitory concentration (MIC) prediction model for Salmonella (Nguyen et al., 2019) (accessed June 13, 2019) was used to predict MIC values for each of the 128 Salmonella isolates in this study, using the assembled genome of each as input. The following were used as dependencies: kmc version 3.0 (Kokot et al., 2017) and XGBoost version 0.82 (Chen and Guestrin, 2016).

\subsection{Prediction of Phenotypic Susceptible-Intermediate-Resistant Classifications Using In Silico Methods}

All 128 Salmonella isolates underwent phenotypic antimicrobial susceptibility testing with a panel of 15 antimicrobials (i.e., amikacin, amoxicillin-clavulanic acid, ampicillin, cefoxitin, ceftiofur, ceftriaxone, chloramphenicol, ciprofloxacin, gentamicin, kanamycin, nalidixic acid, streptomycin, sulfamethoxazole-trimethoprim, sulfisoxazole, and tetracycline) using the Sensititre ${ }^{\circledR}$ system (Trek Diagnostic Systems Ltd., Cleveland, $\mathrm{OH}$ ) available at Cornell University's Animal Health Diagnostic Center as described previously (Rodriguez-Rivera et al., 2014). A "true" (i.e., phenotypic) susceptible-intermediate-resistant (SIR) classification for each of the 15 antimicrobials was obtained for 126 Salmonella isolates by comparing raw MIC values to NARMS breakpoints for Salmonella (accessed March 23, 2020; Supplementary Table S1). For streptomycin, the 1996-2013 NARMS breakpoints were used, as this was compatible with the concentrations used at the time of phenotypic testing (Rodriguez-Rivera et al., 2014). For sulfisoxazole, isolates with MIC $>256$ were classified as 
resistant, as a concentration of $512 \mu \mathrm{g} / \mathrm{mL}$ was not tested. While raw MIC values were unavailable for two isolates (BOV_KENT_16_04-03-08_R8-0967 and ENV_MELA_01_01-10-08_R8-0165; Supplementary Table $\overline{\mathrm{S}} 1$ ), both isolates had previously been categorized as pan-susceptible to all 15 antimicrobials (a classification that was maintained here, as all in silico methods correctly classified these isolates as pan-susceptible).

Supplemental Table S4 of the AMRFinder validation paper (Feldgarden et al., 2019) was

To assess the ability of the MIC prediction method implemented in PATRIC3 to predict Salmonella SIR classification (see section "In Silico Prediction of Antimicrobial Minimum Inhibitory Concentration Values" above), predicted MIC values for 14 antimicrobials produced using PATRIC3 were used to predict the SIR status of each of the 128 Salmonella isolates using the same NARMS breakpoints used for phenotypic testing. Azithromycin MICs produced by PATRIC3 were excluded, as azithromycin was not among the 15 antimicrobials used here for phenotypic testing. The ability of PATRIC3 to predict amikacin resistance was also not evaluated, as amikacin is not among the antimicrobials queried by PATRIC3. A confusion matrix was constructed as described above, using predicted SIR classifications derived from predicted MIC values produced by PATRIC3 and NARMS breakpoints. Additionally, the deviation of raw MIC predictions produced by PATRIC3 $\left(M I C_{P A T R I C 3}\right)$ from "true" raw MIC predictions produced using phenotypic testing $\left(M I C_{\text {Phenotypic }}\right)$ in number of dilution factors ( $\left.N_{\text {dilution factors }}\right)$ was assessed using the following equation:

$$
N_{\text {dilution factors }}=\frac{\ln \left(\frac{M I C_{P A T R I C 3}}{M I C_{P h e n o t y p i c}}\right)}{\ln (2)}
$$

where 1 corresponds to the natural logarithm. For example: if PATRIC3 predicted an MIC value of 8 and the "true" MIC value obtained with phenotypic testing was 2 , then $\ln (8 / 2) / \ln (2)=2$; this means that the PATRIC3 prediction of 8 is +2 dilution factors away from the "true" MIC of 2 (as dilution used for MIC are 2 fold serial dilutions, e.g., $2 \mu \mathrm{g} / \mathrm{mL}, 4 \mu \mathrm{g} / \mathrm{mL}, 8 \mu \mathrm{g} / \mathrm{mL}$ ). 


\subsection{Re-testing of Isolates with Highly Incongruent AMR Phenotypes}

Several $(n=21)$ isolates possessed a phenotypic AMR SIR profile which was deemed to be

213 highly incongruent with its predicted in silico AMR profile, regardless of the in silico pipeline/database used (Supplementary Table S4). For example, $S$. Cerro isolate BOV_CERO_35_10-02-08_R8-2685 was resistant to nine antimicrobials but did not harbor any known acquired AMR genes (Supplementary Table S4). Similarly, S. Newport isolate ENV_NEWP_62_03-05-09_R8-3442 itself was pan-susceptible, but harbored multiple acquired

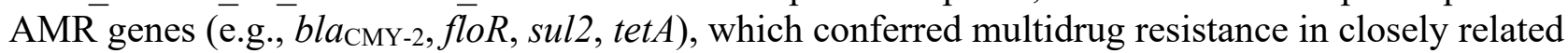
$S$. Newport isolates (Supplementary Table S4). To address these incongruencies, 21 selected Salmonella isolates underwent phenotypic antimicrobial susceptibility re-testing (conducted September 16, 2020) as described above (see section "Prediction of Phenotypic SusceptibleIntermediate-Resistant Classifications Using In Silico Methods"), with the exception of amikacin and kanamycin, as the contemporary panel did not include these antimicrobials (Supplementary Table S4).

Kanamycin testing was conducted separately using a gradient diffusion assay (Jorgensen and Ferraro, 2009) according to the manufacturer's instructions (BioMérieux Kanamycin Strip KM 256, product number 412381). Briefly, bacterial isolates were streaked for single colonies onto Brain Heart Infusion (BHI, Becton Dickinson [BD], Franklin Lakes, NJ, USA) agar plates from frozen glycerol stocks. Precultures were prepared by inoculating a single colony in $3 \mathrm{~mL}$ Mueller-Hinton $(\mathrm{MH})$ broth (BD Difco), followed by incubating at $37^{\circ} \mathrm{C}$ with shaking at $200 \mathrm{rpm}$ for $12-14 \mathrm{~h}$. The precultures were used to inoculate tubes with $5 \mathrm{~mL} \mathrm{MH}$ broth at 1:200 dilution, and the tubes were incubated at $37^{\circ} \mathrm{C}$ with shaking at $200 \mathrm{rpm}$ for 5 hours. Four mL of melted $\mathrm{MH}$ soft agar medium $(0.7 \%$ agar) were mixed with $100 \mu \mathrm{L}$ of culture and poured onto Petri plates containing $15 \mathrm{~mL}$ of $\mathrm{MH}$ agar medium $(0.7 \%$ agar $)$, and the plates were dried for $5 \mathrm{~min}$. Kanamycin gradient strips were laid on top of the soft agar, and the plates were incubated at $35^{\circ} \mathrm{C}$ for 18 hours. MIC values were determined by evaluating the inhibition zone using a magnifying lens according to the manufacturer's instructions.

MIC values obtained from re-testing these isolates were interpreted within NARMS breakpoints as described above (see section "Prediction of Phenotypic Susceptible-IntermediateResistant Classifications Using In Silico Methods") and are reported in the main manuscript (with the exception of amikacin; due to its exclusion from the contemporary panel, original MIC values are reported). Original and updated MIC and SIR values for all 21 isolates are available in Supplementary Table S4.

\subsection{In Silico Plasmid Replicon Detection}

Plasmid replicons were detected in all Salmonella genome assemblies using ABRicate and the PlasmidFinder database (accessed June 11, 2018 via ABRicate's abricate-get_db command). For a plasmid replicon to be considered present in a genome, minimum nucleotide BLAST (blastn) (Camacho et al., 2009) identity and coverage values of 80 and $60 \%$, respectively, were used (Carattoli et al., 2014).

\subsection{Reference-Free SNP Identification and Phylogeny Construction}

A reference-free approach was used to compare the 128 Salmonella genomes sequenced in this study to 442 of the 445 Salmonella genomes described by Worley et al. (Worley et al., 2018); three 252 genomes were omitted because their Sequence Read Archive (SRA) data was not publicly available 
at the time of access (February 20,2019). Raw reads for each of the 442 publicly available genomes

254

were downloaded from SRA (Leinonen et al., 2011; Kodama et al., 2012) and processed and assembled as described above (see section "Whole-Genome Sequencing and Data Pre-Processing" described above). kSNP3 version 3.1 (Gardner and Hall, 2013; Gardner et al., 2015) was used to identify core SNPs among all 570 assembled Salmonella genomes, using the optimal $k$-mer size determined by Kchooser $(k=19)$. IQ-TREE version 1.6.10 (Nguyen et al., 2015) was used to construct a maximum likelihood (ML) phylogeny using the resulting core SNPs and the optimal nucleotide substitution model identified using ModelFinder (determined using model Bayesian Information Criteria [BIC] values); the optimal model was the transversion model with unequal, empirical base frequencies, an ascertainment bias correction, and the FreeRate model with six categories, i.e., the TVM +F+ASC+R6 model (Yang, 1995; Lewis, 2001; Soubrier et al., 2012; Kalyaanamoorthy et al., 2017). Bootstrapping was performed using 1,000 replicates of the Ultrafast Bootstrap method (Minh et al., 2013; Hoang et al., 2018). The resulting ML phylogeny was annotated in $\mathrm{R}$ using the bactax R package (Carroll et al., 2020b) and the following dependencies: ape (Paradis et al., 2004; Paradis and Schliep, 2019), dplyr (Wickham et al., 2020), ggtree (Yu et al., 2017; Yu et al., 2018), phylobase (R Hackathon, 2019), phytools (Revell, 2012), and reshape2 (Wickham, 2007).

\subsection{Pan-Genome Characterization}

GFF files produced by Prokka (see section "In Silico Antimicrobial Resistance Determinant Detection" above) were used as input for Roary version 3.12.0 (Page et al., 2015), which was used to identify orthologous gene clusters at a $70 \%$ protein BLAST (blastp) identity threshold. The resulting gene presence/absence matrix produced by Roary was used as input for besPLOT (https://github.com/lmc297/besPLOT) (Carroll et al., 2020a), which was used to perform non-metric multidimensional scaling (NMDS) (Kruskal, 1964) and construct plots in two dimensions using a Jaccard distance metric and the following dependencies in R: vegan version 2.5-6 (Oksanen et al., 2019), shiny version 1.4.0.2 (Chang et al., 2020), ggplot2 version 3.3.0 (Wickham, 2016), plyr version 1.8.6 (Wickham, 2011), dplyr version 0.8.5 (Wickham et al., 2020), cluster version 2.1.0 (Maechler et al., 2019), and ggrepel version 0.8.2 (Slowikowski, 2020).

Clustering based on gene presence/absence was assessed for each of the following grouping factors: (i) serotype, (ii) farm, and (iii) isolation source (i.e., bovine or farm environmental). For each of the three grouping factors, the following three statistical tests were performed, using the gene presence/absence matrix produced by Roary, a Jaccard distance metric, and 10,000 permutations: (i) the permutest and betadisper functions in R's vegan package (Oksanen et al., 2019) were used to conduct an ANOVA-like permutation test (Anderson, 2006) to test if group dispersions were homogenous (referred to hereafter as the PERMDISP2 test); (ii) analysis of similarity (ANOSIM) (Clarke, 1993) using the anosim function in the vegan package in $\mathrm{R}$ was used to determine if the average of the ranks of within-group distances was greater than or equal to the average of the ranks of between-group distances (Anderson and Walsh, 2013); (iii) permutational analysis of variance (PERMANOVA) (Anderson, 2001) using the adonis2 function in the vegan package in $\mathrm{R}$ was used to determine if group centroids were equivalent. For all tests, a Bonferroni correction was applied to correct for multiple comparisons.

Potential clustering based on AMR gene presence/absence was additionally assessed for the same three grouping factors (serotype, farm, and isolation source), using the presence and absence of AMR determinants detected by AMRFinderPlus as input (i.e., AMR and stress response determinants 
identified using the "plus" option in AMRFinderPlus). All steps were performed as described above, and a Bonferroni correction was used to correct for multiple comparisons.

\subsection{Reference-Based Core SNP Identification Within Serotypes}

For each individual serotype, core SNPs were identified among genomes assigned to that serotype using a reference-based approach. For each serotype, Snippy version 4.3.6 (https://github.com/tseemann/snippy) (Seemann, 2019b) was used to identify core SNPs among all representatives assigned to the serotype, using the trimmed Illumina paired-end reads of each genome as input (see section "Whole-Genome Sequencing and Data Pre-Processing" above), one of six highquality assembled genomes from isolates in this study as a reference genome (Supplementary Table S1), and the following dependencies: BWA MEM version 0.7.13-r1126 (Li and Durbin, 2009; Li, 2013), Minimap2 version 2.15 (Li, 2018), SAMtools version 1.8 (Li et al., 2009), BEDtools version 2.27.1 (Quinlan and Hall, 2010; Quinlan, 2014), BCFtools version 1.8 (Li, 2011), FreeBayes version v1.1.0-60-gc15b070 (Garrison and Marth, 2012), vcflib version v1.0.0-rc2 (Cleary et al., 2015), vt version 0.57721 (Tan et al., 2015), SnpEff version 4.3T (Cingolani et al., 2012), samclip version 0.2 (Seemann, 2019a), seqtk version 1.2-r102-dirty (Li, 2019), and snp-sites version 2.4.0 (Page et al., 2016). Gubbins version 2.3.4 (Croucher et al., 2015) was used to identify and remove recombination within the full alignment that resulted, and the filtered alignment produced by Gubbins was queried using snp-sites to produce an alignment of core SNPs for each serotype.

\section{5}

\subsection{Construction of Within-Serotype Phylogenies}

For each serotype, IQ-TREE version 1.6.10 was used to construct a ML phylogeny, using core SNPs detected among all isolates assigned to the serotype as input (see "Reference-Based Core SNP Identification Within Serotypes" section above), the optimal ascertainment bias-aware nucleotide substitution model selected using ModelFinder, and 1,000 replicates of the UltraFast bootstrap approximation. The temporal structure of each resulting ML phylogeny was assessed using the $R^{2}$ value produced by the best-fitting root in TempEst version 1.5.1 (Supplementary Table S5) (Rambaut et al., 2016).

A tip-dated phylogeny was then constructed for each serotype using BEAST version 2.5.0 (Bouckaert et al., 2014; Bouckaert et al., 2019), the appropriate core SNP alignment, an initial clock rate of $2.79 \times 10^{-7}$ substitutions/site/year (Leekitcharoenphon et al., 2016), and an ascertainment bias correction to account for the use of solely variant sites (Bouckaert, 2014). The modelTest function in R's phangorn package (Schliep, 2011) was used to select an appropriate nucleotide substitution model for each serotype, and the implementation of the model with the lowest BIC value in BEAST 2's SSN package (Bouckaert and Xie, 2017) was selected. For each serotype, combinations of either a strict or lognormal relaxed molecular clock (Drummond et al., 2006) and either a coalescent constant or coalescent Bayesian Skyline (Drummond et al., 2005) population model were tested. For all models, a lognormal prior was used for the clockRate/ucldMean parameters (in real space, $\mathrm{M}=$ $2.058 \times 10^{-6}$ and $\mathrm{S}=2.0$, yielding a median value of $2.79 \times 10^{-7}$ substitutions/site/year, and $2.5 \%$ and $97.5 \%$ quantiles of $5.53 \times 10^{-9}$ and $1.40 \times 10^{-5}$ substitutions/site/year, respectively). For each molecular clock and population model combination for each serotype, three independent stepping stone sampling analyses (Xie et al., 2011) were performed with BEAST 2, using 10 steps of at least 100 million generations. Bayes factors (Kass and Raftery, 1995) were calculated to determine the optimal combination of molecular clock and population model for each serotype (Supplementary Table S5). Ten independent BEAST 2 runs were then performed using the optimal model for each serotype, using chain lengths of at least 100 million generations, sampling every 10,000 generations. Tracer 
version 1.7.1 (Rambaut et al., 2018) was used to ensure adequate mixing of each independent run. The resulting log and tree files were aggregated using LogCombiner-2, and TreeAnnotator-2 (Heled and Bouckaert, 2013) was used to produce a maximum clade credibility tree using $10 \%$ burn-in. The phylogenies were annotated using $\mathrm{R}$ and the following packages: ggplot2, ggtree, and phylobase. Bayesian skyline plots for all serotype groups analyzed using BEAST2 are also available (Supplementary Figure S1); however, due to the limited number of available isolates surveyed among each serotype and the short temporal range queried here, potential changes in effective population sizes may not be robust and are thus not discussed in this study.

\subsection{Data Availability}

Illumina reads are available for all isolates sequenced in this study under NCBI Bioproject Accession PRJNA756552. NCBI BioSample accession numbers for each individual isolate, as well as all associated metadata and genome quality statistics, are available in Supplementary Table S1. All BEAST 2 XML files used for temporal phylogeny construction are available at https://github.com/lmc297/zru_farms.

\section{Results}

\subsection{In silico serotyping of bovine-associated Salmonella resolves incongruencies between traditional serotyping and whole-genome phylogeny}

A total of 128 Salmonella strains isolated from healthy (i.e., subclinically infected) dairy cattle $(n=39)$ and their associated farm environments $(n=89)$ on 13 different New York State farms underwent WGS (Supplementary Table S1). In addition to undergoing traditional serotyping in a laboratory setting, all isolates were assigned serotypes in silico using both (i) SISTR and (ii) SeqSero2 (Supplementary Table S1). Importantly, serotypes assigned in silico using SISTR and/or SeqSero2 were able to resolve all un-typable and/or ambiguous serotypes assigned using traditional serotyping (Supplementary Table S1). Furthermore, in silico serotypes assigned using (i) SISTR's core-genome multi-locus sequence typing (cgMLST) approach and (ii) SeqSero2 were both highly congruent with the Salmonella whole-genome phylogeny (Figure 1) and highly concordant with each other: 127 of 128 (99.2\%) Salmonella isolates sequenced in this study were assigned to identical in silico serotypes using both SISTR cgMLST and SeqSero2 (Supplementary Table S1), with 100\% concordance observed for six of seven observed in silico serotype groups (i.e., $S$. Anatum, $S$. Cerro, $S$. Meleagridis, $S$. Minnesota, $S$. Newport, and $S$. Typhimurium and its variants, assigned to $n=15$, $13,20,1,16$, and 27 isolates, respectively). Among $S$. Kentucky $(n=36)$, a single incongruent isolate was observed (ENV_KENT_16_12-04-07_R8-0061), as SeqSero2 could not detect an O-antigen within the genome and was thus unable to assign this isolate to any serotype. This isolate was assigned a serotype of 8,20:-:z6 using traditional serotyping $(S$. Kentucky has antigenic formula 8,20:i:z6); SISTR classified the isolate as $S$. Kentucky, and the isolate clustered among the $S$. Kentucky isolates sequenced in this study within the Salmonella whole-genome phylogeny (Figure 1 and Supplementary Table S1).

When variants of the $S$. Typhimurium serotype $(n=27)$ were considered, discrepancies were observed between traditional serotype assignments and both in silico methods (Supplementary Table S1). While SeqSero2 could differentiate between $S$. Typhimurium and the O5-variant of $S$. Typhimurium (also known as $S$. Typhimurium variant Copenhagen; "S. Typhimurium Copenhagen" is used hereafter), SISTR was unable to differentiate the two (Supplementary Table S1), as noted previously (Ibrahim and Morin, 2018; Zhang et al., 2019). However, $S$. Typhimurium and $S$. Typhimurium Copenhagen serotype assignments obtained using SeqSero2 and traditional serotyping 
385

386

387

388

389

390

391

392

393

394

395

396

397

398

399

400

401

402

403

404

405

406

407

408

409

410

411

412

413

414

415

416

417

418

419

420

421

422

423

424

425

426

427

428

did not always agree, as five of $27 S$. Typhimurium $/ S$. Typhimurium Copenhagen assignments $(18.5 \%)$ differed between the two methods (Supplementary Table S1). For four of the five incongruent isolates, SeqSero2 assigned an isolate to $S$. Typhimurium Copenhagen, while traditional serotyping assigned a serotype of $S$. Typhimurium; for one isolate, the opposite scenario applied (Supplementary Table S1). Furthermore, the lineages formed by isolates classified here as $S$. Typhimurium Copenhagen using either traditional serotyping or SeqSero2, as well as two $S$. Typhimurium Copenhagen genomes from a previous study (Worley et al., 2018), were polyphyletic (Figure 1); consequently, the whole-genome phylogeny could not be used to reliably differentiate these two variants.

For the remainder of this study, serotypes assigned consistently with at least two out of the three methods (i.e., traditional serotyping, SeqSero2, and SISTR cgMLST) were selected as the final serotype to be reported for each isolate. Nine of the 13 farms surveyed here harbored Salmonella isolates that belonged to a single serotype, while two farms harbored two serotypes or serotype variants (Farms 25 and 35 harbored Typhimurium/Typhimurium Copenhagen and Cerro/Newport, respectively; Supplementary Table S1). The remaining two farms harbored three Salmonella serotypes (Farms 17 and 62 harbored Kentucky/Newport/Typhimurium and Cerro/Minnesota/Newport, respectively; Supplementary Table S1).

\subsection{In silico methods predict antimicrobial susceptibility and resistance among bovine- associated Salmonella with high accuracy and concordance}

Using a 15-antimicrobial panel and NARMS breakpoints for Salmonella, more than half of all isolates in this study ( 81 of $128 ; 63.3 \%$ ) were classified as susceptible to all 15 antimicrobials tested, while 38 isolates $(29.7 \%)$ were classified as resistant to two or more antimicrobials (obtained after the 15-antimicrobial panel was re-run for 22 isolates to resolve discrepancies between in silico predictions and phenotypic AMR data; Supplementary Tables S1 and S4).

Regardless of choice of AMR determinant detection pipeline and AMR determinant database, all pipeline/database combinations performed nearly identically when given the task of predicting phenotypic AMR susceptibility/resistance to 15 antimicrobials using known AMR determinantphenotype associations (Figure 2, Table 1, and Supplementary Tables S2 and S3). Furthermore, all pipeline/database combinations showed an extremely high degree of concordance $(98.0 \%$ or greater for all pipeline/database combinations; Supplementary Figure S2). The overall accuracy of all in silico AMR determinant detection pipeline/database combinations ranged from $95.8-97.4 \%$, with the SRST2 AMR detection tool/ARG-ANNOT AMR determinant database combination achieving the highest accuracy for this data set (Figure 2, Table 1, and Supplementary Table S3). The ARIBA/CARD pipeline/database combination achieved the highest specificity, although all pipeline/database combinations were able to predict phenotypic AMR with high specificity $(>99.0 \%$; Figure 2, Table 1, and Supplementary Table S3). Sensitivity ranged from 71.8-84.4\%, with SRST2 achieving the highest sensitivities $(84.4$ and $84.0 \%$ for the ARG-ANNOT and ResFinder databases, respectively; Table 1 and Supplementary Table S3).

For the AMR determinant detection pipelines that relied on nucleotide BLAST (i.e., ABRicate and BTyper), a range of minimum percent nucleotide identity and coverage thresholds were additionally tested (i.e., all combinations of 50-100\% nucleotide identity in increments of 5\% and $0-100 \%$ coverage in increments of $10 \%$; Supplementary Figure S3) so that the optimal combination(s) could be established for the isolate genomes sequenced here. For ABRicate/ARGANNOT, ABRicate/NCBI, and ABRicate/ResFinder, maximum accuracy was achieved using

This is a provisional file, not the final typeset article 
minimum coverage thresholds of 60,50 , and 50-60\%, respectively, and 75-95\% nucleotide identity thresholds (Supplementary Figure S3). For ABRicate/CARD, minimum thresholds of $60 \%$ coverage and 75\% nucleotide identity were optimal (Supplementary Figure S3). For BTyper/ARG-ANNOT, maximum accuracy was achieved using 60\% coverage and 50-95\% nucleotide identity; for BTyper/MEGARes, 50-60\% coverage and 95\% nucleotide identity were the optimal thresholds

434 (Supplementary Figure S3).

The performance of the PATRIC3 in silico MIC prediction method was additionally evaluated (Figure 3 and Supplementary Figure S4). PATRIC3 was able to correctly classify Salmonella isolates as susceptible-intermediate-resistant (SIR) based on NARMS breakpoints with an overall accuracy of $92.9 \%$ (95\% confidence interval 91.6-94.1\%, accuracy $P$-value [accuracy $>$ no information rate] < 1.25E-26; Figure 3). At the individual antimicrobial level, PATRIC3 achieved $>90 \%$ SIR prediction accuracy for 12 of 14 antimicrobials; only sulfisoxazole and tetracycline resistance prediction accuracies were $<90 \%$ (83.6 and 68.0\%, respectively; Figure 3 and Supplementary Figure S4).

\section{3}

\subsection{Genomic AMR determinants of bovine-associated Salmonella are serotype-associated}

Based on the presence and absence of pan-genome elements among all 128 Salmonella isolates sequenced here, the Salmonella pan-genome was more similar within serotype and within farm than between serotype and between farm, respectively (PERMANOVA and ANOSIM $P<0.05$ after a Bonferroni correction; Figure 4 and Table 2), with serotypes showing a higher degree of pan-genome dissimilarity (ANOSIM $R=0.99$ ) and accounting for a larger proportion of the variance (PERMANOVA $R^{2}=0.93$ ) than farms (Figure 4 and Table 2); however, dispersion among both serotypes and farms differed (PERMDISP2 $P<0.05$ after a Bonferroni correction; Table 2), indicating that the ANOSIM and/or PERMANOVA tests could potentially be confounding dispersion with serotype/farm. Additionally, subclinical bovine Salmonella isolates did not significantly differ from strains isolated from the associated farm environment based on pan-genome element presence/absence (PERMANOVA, ANOSIM, and PERMDISP2 $P>0.05$ after a Bonferroni correction; Figure 4 and Table 2).

Based on the presence and absence of AMR and stress response determinants detected among all 128 Salmonella genomes, isolates were more similar within serotype than between serotypes (PERMANOVA and ANOSIM $P<0.05$ and PERMDISP2 $P>0.05$ after a Bonferroni correction; Figure 4 and Table 2). Additionally, isolates were more similar within farm than between farm based on their AMR and stress response gene presence/absence profiles (PERMANOVA and ANOSIM $P<$ 0.05 ; Figure 4 and Table 2), although significant, potentially confounding dispersion differences among farms were present (PERMDISP2 $P<0.05$; Table 2). As was the case with the pan-genome in its entirety, subclinical bovine Salmonella isolates did not significantly differ from farm environmental isolates based on AMR and stress response gene presence/absence (PERMANOVA, ANOSIM, and PERMDISP2 $P>0.05$ after a Bonferroni correction; Figure 4 and Table 2).

\subsection{Each of two New York State dairy farms harbors a unique, bovine-associated $S$. Anatum lineage}

Fifteen $S$. Anatum strains encompassing four PFGE types (Supplementary Table S1) were isolated from subclinical bovine sources and their associated farm environments on two different New York State dairy farms (i.e., Farms 39 and 56; Figure 5 and Table 3). Notably, the $S$. Anatum lineages circulating on each farm were distinct at a genomic level, with isolates from each farm forming a separate clade (posterior probability $[\mathrm{PP}]=1$ for each; Figure 5). The two farm-associated 
lineages were predicted to share a common ancestor circa 1836 (node age 1836.28 using median node heights; Figure 5), although the age of the common ancestor could not be dated reliably (node height 95\% highest posterior density [HPD] interval 540.85-1978.42; Supplementary Figure S5).

$S$. Anatum isolates from Farm 39 shared a common ancestor circa 2005 (node age 2004.69, node height 95\% HPD 1978.46-2007.69; Figure 5 and Supplementary Figure S5). All Farm 39 S. Anatum isolates possessed identical AMR/stress response gene profiles, and all isolates were pansusceptible except for a single isolate that was resistant to ampicillin (Figure 5). All Farm $39 S$. Anatum isolates additionally harbored ColRNAI plasmids; a single isolate additionally harbored an IncI1 plasmid that appeared to harbor no AMR genes (Figure 5).

$S$. Anatum isolates from Farm 56, however, were considerably more diverse than their Farm 39 counterparts; while a clade containing six of seven strains shared a very recent common ancestor (node age 2006.16, node height 95\% HPD 1985.37-2008.11; Figure 5 and Supplementary Figure S5), a unique lineage represented by a single environmental isolate (ENV_ANAT_56_06-12-08_R81402) was present among $S$. Anatum from Farm 56 (Figure 5). All $S$. Anatum isolates from Farm 56 were predicted to have evolved from a common ancestor that existed circa 1895 (node age 1895.03), although this node could not be reliably dated (node height 95\% HPD 1036.89-1989.944; Supplementary Figure S5). Additionally, $S$. Anatum isolated from Farm 56 showcased a greater degree of AMR heterogeneity than those from Farm 39 (Figure 5). Notably, the isolate comprising the unique Farm $56 S$. Anatum lineage possessed an IncI1 plasmid and bla $a_{\mathrm{CMY}-2}$ and was multidrug resistant (MDR; resistant to amoxicillin-clavulanic acid, ampicillin, cefoxitin, ceftiofur, and ceftriaxone; Figure 5). Three of six $S$. Anatum strains comprising the major Farm $56 S$. Anatum lineage were pan-susceptible. The remaining three isolates were resistant to one of (i) tetracycline, (ii) streptomycin, or (iii) ceftiofur and sulfisoxazole; the streptomycin-resistant isolate additionally exhibited reduced susceptibility to chloramphenicol (Figure 5). The tetracycline-resistant isolate additionally possessed both ColpVC and IncIlplasmids and harbored tetracycline resistance gene tet $C$ (Figure 5). While these data suggest some $S$. Anatum lineages queried here have recently acquired AMR, the limited number of isolates and the large degree of uncertainty for some phylogeny node ages preclude reliable estimation of AMR acquisition timeframes.

\section{1}

\subsection{A closely related $S$. Cerro lineage spans two New York State dairy farms}

Thirteen $S$. Cerro strains encompassing two PFGE types (Supplementary Table S1) isolated from two dairy farms (12 from Farm 62 and one from Farm 35) were found to share a high degree of genomic similarity; isolates differed by, at most, 12 core SNPs and evolved from a common ancestor that existed circa March 2008 (node age 2008.21, common ancestor [CA] node height 95\% HPD interval 2007.6-2008.6; Figure 6, Table 3, Supplementary Figure S6, and Supplementary Table S5). While IncI1 and ColRNAI plasmid replicons were detected in all $13 \mathrm{~S}$. Cerro isolates, only one isolate was not pan-susceptible (Figure 6). Notably, the isolate from Farm 35 (BOV_CERO_35_1002-08_R8-2685) was classified as resistant to nine antimicrobials using phenotypic methods (i.e., amoxicillin-clavulanic acid, ampicillin, cefoxitin, ceftiofur, ceftriaxone, chloramphenicol, streptomycin, sulfisoxazole, and tetracycline); based on the most parsimonious explanation for AMR acquisition, this lineage acquired AMR after July 2008 (node age 2008.51, CA node height 95\% HPD interval 2008.14-2008.75; Figure 6 and Supplementary Figure S6). However, no genomic determinants known to confer resistance to these antimicrobials were detected in the genome of the MDR isolate (Figure 6), and the MDR phenotype was confirmed in a second, independent phenotypic AMR test (Supplementary Table S4). 


\subsection{S. Kentucky strains isolated across five different New York State dairy farms evolved} from a common ancestor that existed circa 2004

Thirty-six $S$. Kentucky isolates encompassing two PFGE types (Supplementary Table S1) isolated across five New York State dairy farms (i.e., five, seven, nine, seven, and eight isolates from each of Farm 14, 16, 17, 19, and 42, respectively) were similar at a genomic level; isolates differed by between 0 and 30 core SNPs and shared a common ancestor that was predicted to have existed circa January/February 2004 (node age 2004.07, CA node height 95\% HPD interval 2000.73-2006.8; Figure 7, Table 3, Supplementary Figure S7, and Supplementary Table S5). Two farms harbored a total of three $S$. Kentucky isolates, which were not pan-susceptible (two isolates from Farm 16 and one from Farm 17; Figure 7). Farm 17 harbored a tetracycline-resistant isolate (ENV_KENT_17_0311-08_R8-0815), which possessed an IncI1 plasmid and tetC (Figure 7). The lineage represented by this isolate was predicted to have acquired tetracycline resistance after March 2007 (node height 2007.19, CA node height 95\% HPD interval 2006.43-2007.84; Figure 7 and Supplementary Figure S7). The two $S$. Kentucky isolates from Farm 16 additionally showed reduced susceptibility to chloramphenicol, a trait predicted to have been acquired by these lineages after December 2006/January 2007 (for the lineage represented by isolate ENV_KENT_16_12-04-07_R8-0061; node height 2006.98, CA node height 95\% HPD interval 2005.95-2007.85) and May 2007 (for the lineage represented by isolate BOV_KENT_16_02-13-08_R8-0838; node height 2007.38, CA node height 95\% HPD interval 2006.59-2008.10; Figure 7 and Supplementary Figure S7). No corresponding genes that may encode for reduced chloramphenicol susceptibility were identified in these two isolates.

\subsection{A clonal $S$. Meleagridis lineage is distributed across two New York State dairy farms and encompasses isolates carrying bla $a_{\mathrm{CTX}-\mathrm{M}-1}$}

Nineteen $S$. Meleagridis isolates encompassing two PFGE types (Supplementary Table S1) were isolated from two dairy farms (13 and six isolates from Farms 01 and 11, respectively) and were highly clonal: isolates differed by fewer than ten core SNPs and evolved from a common ancestor that existed circa May/June 2007 (node age 2007.42, CA node height 95\% HPD interval 2006.912007.75; Figure 8, Table 3, Supplementary Figure S8, and Supplementary Table S5). All but three branches within the $S$. Meleagridis phylogeny had low support ( $\mathrm{PP} \leq 0.41$; Figure 8 and Supplementary Figure S8), indicating that most nodes were unreliable, likely due to the isolates being highly clonal. All $S$. Meleagridis isolates from Farm 11 were pan-susceptible, possessed no plasmid replicons, and did not possess any acquired AMR genes (Figure 8). Among the $S$. Meleagridis isolates from Farm 01, one isolate (ENV_MELA_01_10-02-07_R6-0938) was resistant to ampicillin, ceftiofur, and ceftriaxone, and possessed an IncN plasmid, macrolide resistance gene $m p h(A)$, and beta-lactamase bla $a_{\mathrm{CTX}-\mathrm{M}-1}$ (Figure 8). Two additional $S$. Meleagridis isolates from Farm 01 each exhibited reduced susceptibility to either (i) cefoxitin, sulfisoxazole, and tetracycline, or (ii) ceftiofur (Figure 8).

\subsection{Kanamycin resistance among each of three New York State dairy farms harboring a distinct, multidrug-resistant $S$. Newport lineage is farm-associated}

Sixteen $S$. Newport isolates encompassing three PFGE types (Supplementary Table S1) were isolated from one of three farms (four, five, and seven isolates from Farms 17, 35, and 62, respectively); all isolates were resistant to amoxicillin-clavulanic acid, ampicillin, cefoxitin, ceftiofur, ceftriaxone, streptomycin, sulfisoxazole, and tetracycline (Figure 9). All $S$. Newport genomes harbored IncA/C2 and ColRNAI plasmids, as well as streptomycin resistance genes $A P H\left(3^{\prime \prime}\right)-I b$ and

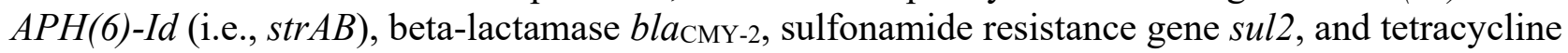


resistance gene tet $A$ (Figure 9). Notably, the $S$. Newport lineage circulating on each farm formed one of three separate clades $(\mathrm{PP}=0.99-1.0)$ that evolved from a common ancestor that existed circa March/April 2004 (node age 2004.23, CA node height 95\% HPD interval 2000.42-2007.85; Figure 9, Table 3, Supplementary Figure S9, and Supplementary Table S5).

The $S$. Newport lineages present on Farm 17 and Farm 62 were additionally resistant to chloramphenicol and kanamycin and possessed chloramphenicol and kanamycin resistance genes floR and $A P H\left(3^{\prime}\right)-I a$, respectively (Figure 9). The Farm 17 and Farm 62 lineages evolved from a common ancestor predicted to have existed circa November/December 2005 (node age 2005.91, CA node height 95\% HPD interval 2003.77-2007.85; Figure 9 and Supplementary Figure S9). All members of the Farm 17 lineage additionally harbored a ColpVC plasmid and shared a common ancestor dated to circa August/September 2007 (node age 2007.65, CA node height 95\% HPD interval 2007.29-2007.85; Figure 9 and Supplementary Figure S9). The Farm 62 lineage, which did not possess the ColpVC plasmid, evolved from a common ancestor circa August/September 2008 (node age 2008.68, CA node height 95\% HPD interval 2008.42-2008.78; Figure 9 and Supplementary Figure S9).

Unlike the $S$. Newport lineages present on Farm 17 and Farm 62, the Farm $35 S$. Newport lineage did not possess kanamycin resistance gene $A P H\left(3^{\prime}\right)$-Ia and was kanamycin-susceptible (Figure 9). The common ancestor of the Farm 35 S. Newport lineage was dated circa May 2008 (node age 2008.38, CA node height 95\% HPD interval 2008.06-2008.55). All but one Farm 35 S. Newport isolates were additionally resistant to chloramphenicol and possessed floR; BOV_NEWP_35_10-0208_R8-2688 did not possess floR and was chloramphenicol-susceptible (Figure 9).

\subsection{Each of four major lineages composed of $S$. Typhimurium and its O5-Copenhagen variant is associated with one of three New York State dairy farms}

Twenty-seven bovine and farm environmental $S$. Typhimurium and $S$. Typhimurium Copenhagen isolates that encompassed five PFGE types (Supplementary Table S1) were isolated from three dairy farms (1, 10, and 16 strains isolated from Farm 17, 22, and 25, respectively). All isolates queried here shared a common ancestor that existed circa 1936 (node age 1935.62, CA node height 95\% HPD interval 1864.84-1991.86; Figure 10, Table 3, Supplementary Figure S10, and Supplementary Table S5). Notably, the $S$. Typhimurium Copenhagen variant was polyphyletic (Figure 10), regardless of whether traditional or in silico (i.e., SeqSero2) methods had been used for serotype variant assignment. Additionally, the $S$. Typhimurium/S. Typhimurium Copenhagen isolates sequenced here showcased the most diverse AMR phenotypic profiles and AMR gene presence/absence profiles (Figures 4 and 10).

Isolates from Farm 25 were partitioned into two clades: one containing $S$. Typhimurium isolates, and one containing $S$. Typhimurium Copenhagen isolates (based on SeqSero2's in silico serotype assignments; Figure 10). Farm 25 isolates assigned to the $S$. Typhimurium Copenhagen variant (i) shared a common ancestor that existed circa December 2007/January 2008 (node age 2007.99, CA node height 95\% HPD interval 2007.68-2008.21); (ii) were all resistant to ampicillin, kanamycin, streptomycin, sulfisoxazole, and tetracycline, with reduced susceptibility to additional antimicrobials observed sporadically; (iii) all possessed replicons for IncA/C2, IncFIB(AP001918), and IncFII(s) plasmids; and (iv) all possessed streptomycin resistance genes aadA12, $A P H\left(3^{\prime \prime}\right)-I b$ and $A P H(6)-I d$ (i.e., str $A B$ ), beta-lactamase $b l_{\mathrm{TEM}-1}$, and antiseptic resistance gene qacE delta 1, with other AMR/stress response genes present sporadically (Figure 10 and Supplementary Figure S10). Farm 25 isolates assigned to the $S$. Typhimurium clade shared a common ancestor that existed circa 
606

607

608

609

610

611

612

613

614

615

616

617

618

619

620

621

622

623

624

625

626

627

628

629

630

631

632

633

634

635

636

637

638

639

640

641

642

643

644

645

646

647

648

649

July 2007 (node age 2007.55, CA node height 95\% HPD interval 2007.18-2007.78; Figure 10 and Supplementary Figure S10). All Farm 25 S. Typhimurium isolates were resistant to cefoxitin; resistance to additional antimicrobials, along with presence of IncI1 plasmids and bla $a_{\mathrm{CMY}-2}$, was observed sporadically (Figure 10).

The isolate from Farm 17 was predicted to belong to the $S$. Typhimurium Copenhagen serotype variant using SeqSero2 and shared a common ancestor with the $S$. Typhimurium isolates from Farm 22, which existed circa 2000 (node age 1999.92, CA node height 95\% HPD interval 1991.72-2006.21; Figure 10 and Supplementary Figure S10). Of the ten $S$. Typhimurium strains from Farm 22, seven were pan-susceptible (Figure 10). A bovine strain

(BOV_TYPH_22_03-14-08_R8-0865) was resistant to ampicillin, ceftiofur, and ceftriaxone and

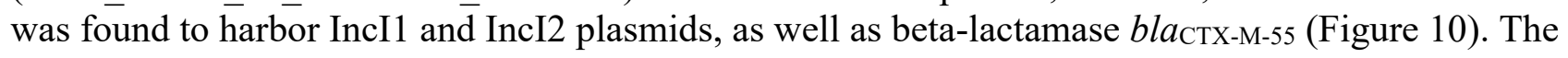
remaining two bovine isolates were intermediately resistant to chloramphenicol and additionally resistant to either (i) amoxicillin-clavulanic acid, ampicillin, and sulfisoxazole, or (ii) tetracycline (Figure 10). Overall, isolates from Farm 17 and Farm 22 shared a common ancestor with the Farm 25 $S$. Typhimurium clade that existed circa 1988 (node age 1988.02, CA node height $95 \%$ HPD interval 1969.24-2002.47; Figure 10 and Supplementary Figure S10).

\section{Discussion}

\subsection{WGS can be used to monitor pathogen microevolution and temporal AMR dynamics in animal reservoirs}

Cattle may act as a reservoir for Salmonella and may facilitate its transmission to other animals (Mentaberre et al., 2013; Wiethoelter et al., 2015) or humans, either through direct contact or via the food supply chain (Hoelzer et al., 2011; Cummings et al., 2012; Mughini-Gras et al., 2014; An et al., 2017; Gutema et al., 2019). Even outside of a bovine host, Salmonella can survive in the farm environment for a prolonged amount of time, making persistent strains a particularly relevant threat to animal and human health (Rodriguez et al., 2006; Cummings et al., 2010b; Gorski et al., 2011; Toth et al., 2011; Tassinari et al., 2019). This threat can be compounded when persistent strains are exposed to antimicrobials, as a number of studies have linked antimicrobial exposure to the emergence of AMR in different foodborne pathogens, including Salmonella, Escherichia coli, and Campylobacter (Boerlin et al., 2001; McDermott et al., 2002; Delsol et al., 2003; Dutil et al., 2010; Hoelzer et al., 2017).

However, AMR acquisition among pathogens in livestock environments is far from absolute; in the absence of selective pressures (e.g., antimicrobial exposure), some AMR traits may be associated with a fitness cost for a given organism (Melnyk et al., 2015; Hoelzer et al., 2017; San Millan and MacLean, 2017). Consequently, interventions or changes in farm management practices (e.g., limiting antimicrobial use for all or selected antimicrobials, targeted use of some antimicrobials) may lead to reduced selection of AMR bacteria (Aarestrup, 2015; Tang et al., 2017; Scott et al., 2018). As such, the dynamics of AMR acquisition and loss among livestock-associated bacterial pathogens are complex and influenced by a wide range of factors, including the antimicrobials and treatment regimens used, farm management practices, environmental conditions, and the biology of the pathogens themselves (Aarestrup, 2015; Hoelzer et al., 2017; Davidson et al., 2018; Pereira et al., 2019; Clarke et al., 2020).

Using a WGS-based approach applied to serially sampled Salmonella strains isolated over a short time frame (i.e., less than two years), the study detailed here reveals that sporadic acquisition and loss of acquired AMR genes can occur within closely related populations over a short timescale. 
650

651

652

653

654

655

656

657

658

659

660

661

662

663

664

665

666

667

668

669

670

671

672

673

674

675

676

677

678

679

680

681

682

683

684

685

686

687

688

689

690

691

692

693

One particularly notable observation is represented by multiple, independent acquisitions of the betalactamase bla $a_{\mathrm{CMY}}$ among $S$. Typhimurium and $S$. Typhimurium Copenhagen, as all bla $a_{\mathrm{CMY}}$ acquisition events within this serotype group were confined to the 2000s. bla $a_{\mathrm{CMY}}$ can confer resistance to cephalosporins, including (i) ceftriaxone, which has been used in human medicine since the early 1980s, and is used to treat invasive salmonellosis cases when fluoroquinolones cannot be used (e.g., for pediatric salmonellosis cases), and (ii) ceftiofur, which has been used in veterinary settings since the late 1980s to treat disease cases among dairy cattle and other animals (Hornish and Kotarski, 2002; Alcaine et al., 2005; Liebana et al., 2013; Yang et al., 2016; Carroll et al., 2017b; Carroll et al., 2020a). Because bla ${ }_{\mathrm{CMY}}$ often confers resistance to both ceftriaxone and ceftiofur, there has been concern that the use of ceftiofur in livestock can contribute to the dissemination of bla $a_{\mathrm{CMY}}$ and thus yield bacterial populations that are co-resistant to ceftriaxone (Alcaine et al., 2005; Tragesser et al., 2006; Carroll et al., 2017b; Carroll et al., 2020a).

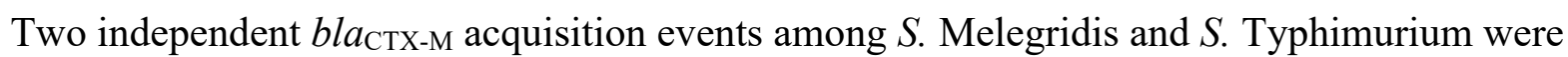
additionally observed. bla $a_{\text {СтХ-м, }}$ which also confers resistance to cephalosporins, was rarely detected in the United States in the 1990s (Lewis et al., 2007; Canton et al., 2012). However, bla $a_{\text {CTX-M }}$ rapidly increased in prevalence in the United States between 2000 and 2005 (Lewis et al., 2007; Canton et al., 2012), and there is evidence that bacterial populations associated with dairy cattle may have been affected as well. In a study of $E$. coli isolated from dairy cattle in the western United States, the prevalence of bla $a_{\text {СтX-м }}$ was found to have increased between 2008 and 2012 (Afema et al., 2018). The results of our study are congruent with these findings, as all observed bla $a_{\mathrm{CTX}-\mathrm{M}}$ acquisition events were estimated to have occurred in the 2000s.

AMR loss events were additionally observed among the bovine-associated, MDR $S$. Newport isolates sequenced here. Prevalence of MDR $S$. Newport among humans increased rapidly in the United States within the late 1990s and early 2000s and was linked to cattle exposure, farm/petting zoo exposure, unpasteurized milk consumption, and ground beef consumption (Spika et al., 1987; Gupta et al., 2003; Karon et al., 2007). While chloramphenicol resistance is often a hallmark characteristic of MDR $S$. Newport, the MDR $S$. Newport lineage represented by an isolate in this study was chloramphenicol-susceptible and was predicted to have lost chloramphenicol resistance gene floR after 2008. These results indicate that even well-established MDR pathogens can still be subjected to temporal changes in AMR profile.

Due to the global burden that AMR pathogens impose on the health of humans and animals, numerous agencies have called for improved monitoring of pathogens and their associated AMR determinants along the food supply chain (World Health Organization, 2014; 2017; Centers for Disease Control and Prevention, 2019). The study detailed here showcases how WGS can be used to identify temporal changes in the resistomes of livestock-associated pathogens at the farm level. However, further sequencing efforts querying (i) a larger selection of Salmonella strains isolated from livestock on individual farms (ii) over a longer timeframe are needed to determine whether the AMR dynamics observed here are merely sporadic, or rather are indications of larger trends.

\subsection{Bovine-associated Salmonella lineages with heterogeneous antimicrobial resistance profiles may be present across multiple farms or strongly farm-associated}

Geography has been shown to play an important role in shaping bacterial populations (Achtman, 2008; Strachan et al., 2015), including some Salmonella lineages (Carroll et al., 2017b; Palma et al., 2018; Fenske et al., 2019; Liao et al., 2020). However, for some foodborne pathogens, including some Salmonella populations, global spread of lineages due to human migration and

This is a provisional file, not the final typeset article 
movement of food and animals can often obfuscate local phylogeographic signals (Wong et al., 2015; Llarena et al., 2016; The et al., 2016; Palma et al., 2018).

In the study detailed here, Salmonella lineages isolated from cattle and their associated environments on 13 separate farms in a confined geographic location (i.e., New York State) were found to vary in terms of the farm-specific signal they possessed; some lineages (i.e., $S$. Anatum, $S$. Newport, $S$. Typhimurium, $S$. Typhimurium Copenhagen, some $S$. Kentucky populations) were found to be strongly associated with a particular farm, while other lineages (i.e., $S$. Cerro, $S$. Meleagridis, some $S$. Kentucky populations) were distributed across multiple farms. Multiple scenarios may explain the existence of Salmonella lineages distributed across multiple farms, including movement of livestock, humans, pets, and/or wildlife (Skov et al., 2008; Hoelzer et al., 2011; Palma et al., 2018) or introduction via feed; however, additional metadata (e.g., farm geography, proximity to other farms in the study, and management practices) are needed to draw further conclusions. Even with limited metadata available, WGS data can provide important insights into Salmonella transmission and introduction on farms, as shown in this study. For example, for one farm (i.e., Farm 25), two Salmonella Typhimurium clonal groups were present (i.e., one representing Typhimurium and one representing Typhimurium Copenhagen), each of which shared a common ancestor dated circa 2007. WGS data can be used to identify time frames in which Salmonella lineages may have emerged in a given farm or region, which could help pinpoint root causes (e.g., changes in management practices that occurred around the predicted time of emergence).

While the characterization of additional, larger strain sets from more geographically diverse farms is essential, our data suggest that specific Salmonella clones may persist on a given farm. This suggests that WGS databases covering isolates from a large number of farms could be used to develop initial hypotheses about farm sources of Salmonella strains. While such applications are tempting, it is crucial that these types of data are only used for initial hypothesis generation; rigorous, critical epidemiological investigations are essential before any conclusions regarding strain source are drawn.

\subsection{In silico serotyping of bovine-associated Salmonella can outperform traditional serotyping}

Well into the genomic era, serotyping remains a vital microbiological assay that allows Salmonella isolates to be classified into meaningful, evolutionary units. Serotype assignments are used to facilitate outbreak investigations and surveillance efforts, construct salmonellosis risk assessment frameworks, and inform food safety and public health policy and decision-making efforts (Yoshida et al., 2016; Gutema et al., 2019). Importantly, serotyping is used worldwide to monitor salmonellosis cases among humans and animals, including cattle (Gutema et al., 2019; Centers for Disease Control and Prevention, 2020).

In this study, serotypes assigned using traditional phenotypic methods were compared to serotypes assigned using two in silico methods (i.e., SISTR and SeqSero2). Notably, both in silico serotyping approaches outperformed traditional Salmonella serotyping for this data set. Serotypes assigned using SISTR's cgMLST approach and/or SeqSero2 were congruent with the Salmonella whole-genome phylogeny and were able to resolve all un-typable, ambiguous, and incorrectly assigned serotypes (Supplementary Table S1). It is essential to note that the data set queried here is far too small and, thus, inadequate to formally benchmark these tools. Furthermore, all serotypes studied here were among the ten most frequently reported serotypes of Salmonella isolated from subclinical cattle between 2000 and 2017 (Gutema et al., 2019), indicating that they are wellrepresented in public databases and thus likely do not pose a significant challenge to in silico tools. 
738

739

740

741

742

743

744

745

746

747

748

749

750

751

752

753

754

755

756

757

758

759

760

761

762

763

764

765

766

767

768

769

770

771

772

773

774

775

776

777

778

779

780

781

782

However, the results observed here reflect results observed in several recent studies querying larger numbers of isolate genomes and/or a wider array of diverse Salmonella serotypes (Yachison et al., 2017; Ibrahim and Morin, 2018; Diep et al., 2019; Banerji et al., 2020; Uelze et al., 2020). In their analysis of 1,624 animal- and food-associated (i.e., non-human) Salmonella isolate genomes assigned to 72 serotypes, Uelze et al. (Uelze et al., 2020) reported that SISTR and SeqSero2 achieved the highest and second-highest accuracy of all tested in silico Salmonella serotype assignment tools, correctly serotyping $94 \%$ and $87 \%$ of isolates, respectively. However, unlike the results observed here, the authors note that neither tool outperformed traditional serotyping conducted by Salmonella reference laboratories. Similarly, in a study of 813 Salmonella isolates, SISTR outperformed the original version of SeqSero (i.e., SeqSero 1.0) with serotype prediction accuracies of $94.8 \%$ and $88.2 \%$, respectively (Yachison et al., 2017).

With WGS data in hand, in silico serotyping is rapid, scalable, inexpensive, and reproducible (Uelze et al., 2020). Nevertheless, it is important to be mindful of the strengths and weaknesses of different in silico serotyping tools. In their benchmarking study, Uelze et al. (Uelze et al., 2020) recommended SISTR as the optimal contemporary tool for routine in silico Salmonella serotyping based on overall accuracy; however, they additionally report that the raw read mapping approach implemented in SeqSero2 (i.e., "allele mode") outperforms SISTR for prediction of monophasic variants. Banerji et al. (Banerji et al., 2020) did not assess the performance of SISTR on their data set, as it requires assembled genomes and not raw reads (another potential drawback if a high-quality assembly is not available or obtainable for an isolate of interest); however, they found that both SeqSero and MLST approaches misidentified monophasic variants, particularly among the important monophasic $S$. Typhimurium lineage. Among the bovine-associated Salmonella strains sequenced here, we observed a combination of $S$. Typhimurium strains that possessed the O5 epitope, and those that did not (i.e., $S$. Typhimurium Copenhagen). Importantly, SISTR was unable to differentiate $S$. Typhimurium from $S$. Typhimurium Copenhagen, while SeqSero2 could, as reported previously (Ibrahim and Morin, 2018; Zhang et al., 2019). While the differentiation of S. Typhimurium from its O5- counterpart may not be essential for all microbiological applications, it is important to be aware of this limitation; $S$. Typhimurium Copenhagen has been responsible for outbreaks and illnesses around the world (Luceron et al., 2017; Tack et al., 2020) and can be multidrug-resistant, as demonstrated here and elsewhere (Frech et al., 2003; Tack et al., 2020).

While serotypes assigned in silico using SISTR and SeqSero2 are highly accurate and congruent, each tool has strengths and limitations; as such, an approach that utilizes both methods, such as the one employed here, may increase accuracy and minimize potential misclassifications. Results from other studies support this (Yachison et al., 2017; Banerji et al., 2020). For example, in an analysis of 520 primarily human-associated Salmonella isolate genomes, Banerji et al. (Banerji et al., 2020) found that serotypes assigned in silico using SeqSero showed 98\% concordance with traditional serotyping and outperformed serotype assignment using seven-gene MLST. However, when SeqSero and seven-gene MLST were used in combination, in silico serotyping accuracy surpassed 99\%, consistent with our results that a combination of SeqSero 2 and cgMLST-based serotyping (as implemented in SISTR) improved in silico serotyping accuracy. Overall, the results provided here lend further support to the idea that in silico serotyping may eventually replace traditional serotyping as WGS becomes more widely used and accessible (Yachison et al., 2017; Banerji et al., 2020; Uelze et al., 2020).

\subsection{Limitations of the in silico AMR method evaluation presented here, and considerations for future AMR monitoring efforts among livestock and beyond}

This is a provisional file, not the final typeset article 
In addition to studying the microevolution and AMR dynamics of bovine Salmonella on a genomic scale, the study presented here compared results obtained from numerous in silico AMR characterization pipelines that attempt to replicate traditional microbiological assays used to characterize AMR Salmonella. More specifically, each of the following tools was applied to the set of 128 bovine-associated Salmonella genomes sequenced here: (i) combinations of five in silico AMR determinant detection pipelines (i.e., ABRicate, AMRFinderPlus, ARIBA, BTyper, and SRST2) and one to five AMR determinant databases (i.e., ARG-ANNOT, CARD, MEGARes, NCBI, and ResFinder); and (ii) an in silico MIC prediction tool (i.e., PATRIC3).

Here, all AMR determinant detection pipelines and AMR determinant databases showed an extremely high degree of concordance; regardless of pipeline or database selection, all tools performed nearly identically on an SIR-prediction task relative to (i) "true" SIR classifications based on NARMS breakpoints and "true" MIC values obtained for a panel of 15 antimicrobials, and (ii) each other. A previous, small-scale ( $n=111)$ WGS-based study of AMR Salmonella observed similarly high rates of concordance among several in silico AMR determinant detection tools (Cooper et al., 2020). However, in addition to its small sample size, this study also relied on Salmonella strains isolated from a single source (broiler chickens) in a single country (Canada) over an extremely short temporal range (December 2012-December 2013). Similarly, the study detailed here is not a formal benchmarking study, and it is essential that its numerous limitations are pointed out.

First and foremost, the study conducted here relied on WGS data from an extremely small sample of Salmonella isolates $(n=128)$ from a single source (dairy cattle and their surrounding farm environments) in a confined geographic area (New York State, United States) isolated over a short temporal range (fewer than two years). While all isolates were "unique" (i.e., each strain was isolated from a separate sampling event of a unique source), many isolates were highly similar at both the genomic and pan-genomic level (e.g., $S$. Cerro, $S$. Meleagridis), indicating that, in some cases, the same lineage was being sampled repeatedly over time. Consequently, this relatively miniscule sample is unrepresentative of AMR pathogens and, more specifically, Salmonella as a whole; readers should not infer the general superiority or inferiority of any AMR detection tool or database tested, and the results obtained here should not be extrapolated to external data sets.

Secondly, the data set queried here was heavily biased towards susceptible isolates. More than half of all isolates were pan-susceptible to the 15 antimicrobials included on the panel, and only 21 unique phenotypic SIR profiles were observed. Congruent with this, relatively little diversity was observed in terms of AMR gene profile (e.g., the AMRFinderPlus pipeline produced 20 unique $\mathrm{AMR} /$ stress response determinant presence/absence profiles among the 128 isolates sequenced here). This is not particularly surprising; numerous studies have shown that the resistomes of bovineassociated Salmonella tend to be less diverse than Salmonella isolated from humans (Afema et al., 2015; Carroll et al., 2017b), as well as some other animals (Mellor et al., 2019). Furthermore, the resistomes of Salmonella isolated from subclinical cattle, such as the isolates queried in this study, have been shown to be less diverse than the resistomes of Salmonella isolated from cattle showing clinical signs of disease (Afema et al., 2015).

The relative homogeneity of the subclinical Salmonella bovine resistome and bias towards antimicrobial-susceptible isolates have important implications for the AMR pipeline/database comparison conducted here. For this data set, stringent and conservative approaches are rewarded, as isolates that do not possess AMR determinants are more likely to be predicted to be susceptible. While it is possible that different AMR detection tools may perform better on WGS data from 
pathogens with more diverse resistomes, very few formal benchmarking studies of in silico AMR determinant detection tools currently exist (Hendriksen et al., 2019). The choice of AMR determinant database in combination with the choice of pipeline, on the other hand, can clearly affect AMR determinant identification in a critical way. For example, the ARG-ANNOT database (Gupta et al., 2014), a manually curated AMR determinant database first published in 2014, is not updated as frequently as other AMR databases (e.g., CARD, NCBI, ResFinder). Since it was last updated in May 2018 (accessed May 25, 2020), at the time of our study, the database does not yet include three novel plasmid-mediated genes $(m c r-8,-9$, and -10$)$ that can confer resistance to colistin, a last-resort antibiotic used to treat MDR and extensively drug resistant infections (Wang et al., 2018; Carroll et al., 2019; Wang et al., 2020). Similarly, versions of tools that rely on even older versions of this database would not be able to detect all members of the continuously growing repertoire of $\mathrm{mcr}$ genes. For the low-diversity subclinical bovine Salmonella resistomes queried here, the use of a smaller database was inconsequential, as reflected in the high congruency of all methods and databases observed here. For some studies, a manually curated database of AMR genes that is updated conservatively may possibly even be desirable, as such a database may yield less noise and improve interpretability and reproducibility. However, for pathogens with more diverse resistomes (e.g., human clinical isolates, isolates from geographic regions with different antibiotic use practices), the omission of critically important genes could be a disastrous flaw. Similar to our results, a large study $(n=6,242)$ querying NARMS isolates belonging primarily to the Salmonella enterica species $(n=5,425)$ observed a high degree of concordance between NCBI's AMRFinder tool and ResFinder (this study was used to validate AMRFinder and the NCBI AMR determinant database) (Feldgarden et al., 2019). However, when differences between tools were observed, the vast majority (81\%) were attributed to differences in database composition (Feldgarden et al., 2019; Hendriksen et al., 2019).

Thirdly, the small sample size $(n=128)$ and sparsity of AMR isolates available in this study limited the methods that could be used to formulate the AMR tool/database comparison. The approach used here was similar to the one used to validate the AMRFinder tool (Feldgarden et al., 2019) in that it relied on known AMR-determinant/AMR phenotype associations available in the literature (see Supplemental Table S4 of Feldgarden, et al.) (Feldgarden et al., 2019). As such, the approach used here does not account for previously unobserved genotype/phenotype associations. Furthermore, different variants of the same AMR gene may yield different AMR phenotypes; for example, some variants of the OXA beta-lactamases are able to confer resistance to cephalosporins, while others are not (Evans and Amyes, 2014). All AMR determinant detection pipelines tested here produced nearly identical genes calls among the 128 isolates sequenced here, and all detected AMR determinants were manually annotated in a consistent fashion; while the overall accuracy of all pipeline/database combinations tested here could likely be optimized if more accurate, data setspecific AMR genotype/phenotype associations could be derived, congruency between tools/databases would likely remain high.

\section{Classifying bacterial pathogens into discrete SIR groups using AMR determinant detection} methods is challenging, as it requires users to have a great degree of prior knowledge regarding the AMR determinants that are detected, the antimicrobials of interest, and the pathogen being studied. PATRIC3's MIC prediction tool (Nguyen et al., 2019) offers a promising departure from this framework, as it allows for the prediction of MIC values directly from WGS data. Interpreting the resulting in silico MIC values does not require any prior knowledge on the user end, and results can be harmoniously integrated into the SIR framework using clinical breakpoints. Among the Salmonella isolates sequenced here, SIR classification using PATRIC3 resulted in an overall accuracy of $93 \%$. However, all of the limitations of this study described above for AMR determinant detection (e.g., small sample size, AMR sparsity bias, single-source, single geographic region, small 
temporal range) apply to in silico MIC prediction as well; for example, when "intermediate" resistance predictions produced via PATRIC3 are re-classified as "susceptible" (as was done for the AMR determinant detection approaches used here), PATRIC3's accuracy for this data set increases to $96.0 \%$ and is on par with all other AMR prediction methods tested here. Readers should thus interpret comparisons between these methods with caution.

Benchmarking and validating AMR detection and prediction tools is notoriously challenging (Feldgarden et al., 2019), and very few researchers have undertaken this task (Hendriksen et al., 2019). While high congruency may be observed between tools (Clausen et al., 2016), identification of a clear "optimal" method for in silico AMR characterization has remained elusive, as the few available benchmarking studies differ in terms of the tools tested, the AMR database(s) used, and the data set(s) chosen for benchmarking. Furthermore, the underlying WGS data can affect pipeline performance (Clausen et al., 2016; Feldgarden et al., 2019). For example, assembly quality has been shown to influence AMR determinant detection for methods that rely on assembled genomes (Clausen et al., 2016; Hendriksen et al., 2018; Hendriksen et al., 2019). Thus, whether a read- or assembly-based method performs optimally can depend on a given data set (e.g., sequencing quality, the organism being studied). Another criticism of BLAST-based AMR gene detection methods among assembled genomes has been the choice of thresholds used for considering AMR determinants present or absent (Hendriksen et al., 2019). Here, no significant differences were observed between the accuracy of read-based ARIBA and SRST2 and assembly-based ABRicate, AMRFinderPlus, and BTyper. Additionally, for BLAST-based methods, a relatively wide range of optimal nucleotide identity and coverage values were found to maximize accuracy, with thresholds of $75 \%$ identity and 50-60\% coverage adequate for most pipeline/database combinations. Overall, when selecting an in silico AMR characterization method, researchers should take into account not only practical considerations (e.g., whether reads or assembled genomes are available, the quality of reads and/or assembled genomes), but also the biology of the pathogen being studied (e.g., by querying organism-specific, AMR-conferring point mutations). To assess the robustness of in silico AMR predictions, researchers may additionally consider employing multiple in silico AMR characterization tools and/or databases in combination, as well as testing various AMR gene detection thresholds.

Finally, it is essential to note that accuracy estimates for in silico AMR characterization tools relative to gold-standard phenotypic methods are only as reliable as the phenotypic data they rely on. Previous studies of Salmonella that attempted to predict phenotypic AMR using in silico methods (McDermott et al., 2016; Cooper et al., 2020) have reported accuracy values between 98 and 100\%. For this data set, the highest accuracy achieved was 97.4\% (for SRST2/ARG-ANNOT). However, sensitivity (i.e., the ability of an in silico pipeline/database combination to correctly classify an isolate as phenotypically resistant to an antimicrobial) was lower for this study (71.8-84.4\%) than sensitivity estimates calculated in a study of MDR Salmonella that included bovine isolates from New York State (97.2\%) (Carroll et al., 2017b). As mentioned above, this could be due to the sparsity of AMR among isolates in this data set (i.e., predicting susceptible, rather than resistant phenotypes, is incentivized here). However, it is important to note that the AMR phenotypes of several isolates were highly incongruent with their respective AMR genotypes, regardless of the tool/database used for in silico AMR prediction. For example, one $S$. Cerro isolate (BOV_CERO_35_10-02-08_R8-2685) was reported to be phenotypically resistant to nine antimicrobials but did not possess any acquired AMR determinants known to produce this phenotypic AMR profile. A recent case study in which WGS and phenotypic methods were used to characterize Salmonella isolates from raw chicken identified numerous AMR genotype/phenotype discrepancies resulting in both false negative and false positive predictions for in silico methods 
922 (Zwe et al., 2020). In this case study, the authors attributed these discrepancies to heteroresistant

923 Salmonella subpopulations (i.e., a subpopulation of bacteria that exhibits a range of susceptibility to a particular antimicrobial). The possibility that several heteroresistant Salmonella populations were characterized here cannot be discounted, as isolates underwent phenotypic AMR characterization and WGS separately (i.e., years apart). Other biological phenomena, such as plasmid loss during storage or culturing, or unknown/undetected resistance genes or mutations, could also contribute to discrepancies (Hendriksen et al., 2018). However, it is also possible that one or more incongruent isolates was mislabeled and/or mishandled during AMR phenotyping, genomic DNA extraction, and/or WGS. While removal of these isolates from the data set would increase overall prediction accuracy, the high congruency between AMR genotyping methods would be unaffected.

\section{Author Contributions}

LMC designed and carried all computational analyses. AJB, AG, JDS, KJC, and RAC collected, analyzed, and/or interpreted all microbiological data. LMC and MW conceived the study and co-wrote the manuscript with input from all authors.

\section{$936 \quad 6 \quad$ Funding}

This material is based on work supported by the National Science Foundation Graduate

Research Fellowship Program under grant no. DGE-1650441.

\section{$7 \quad$ Conflict of Interest}

The authors declare that the research was conducted in the absence of any commercial or financial relationships that could be construed as a potential conflict of interest.

\section{Acknowledgments}

This project was supported in part by the Cornell University Zoonoses Research Units of the Food and Waterborne Diseases Integrated Research Network, funded by the National Institute of Allergy and Infectious Diseases, National Institutes of Health, under contract no. N01- AI-30054.

\section{References}

Aarestrup, F.M. (2015). The livestock reservoir for antimicrobial resistance: a personal view on changing patterns of risks, effects of interventions and the way forward. Philos Trans $R$ Soc Lond B Biol Sci 370, 20140085.

Achtman, M. (2008). Evolution, population structure, and phylogeography of genetically monomorphic bacterial pathogens. Annu Rev Microbiol 62, 53-70.

Afema, J.A., Ahmed, S., Besser, T.E., Jones, L.P., Sischo, W.M., and Davis, M.A. (2018). Molecular Epidemiology of Dairy Cattle-Associated Escherichia coli Carrying blaCTX-M Genes in Washington State. Appl Environ Microbiol 84.

Afema, J.A., Mather, A.E., and Sischo, W.M. (2015). Antimicrobial Resistance Profiles and Diversity in Salmonella from Humans and Cattle, 2004-2011. Zoonoses Public Health 62, 506-517.

Agren, E.C., Wahlstrom, H., Vesterlund-Carlson, C., Lahti, E., Melin, L., and Soderlund, R. (2016). Comparison of whole genome sequencing typing results and epidemiological contact 
information from outbreaks of Salmonella Dublin in Swedish cattle herds. Infect Ecol Epidemiol 6, 31782.

963

Alcaine, S.D., Sukhnanand, S.S., Warnick, L.D., Su, W.L., Mcgann, P., Mcdonough, P., and Wiedmann, M. (2005). Ceftiofur-resistant Salmonella strains isolated from dairy farms represent multiple widely distributed subtypes that evolved by independent horizontal gene transfer. Antimicrob Agents Chemother 49, 4061-4067.

966

967

968

969

Anderson, M.J. (2001). A new method for non-parametric multivariate analysis of variance. Austral Ecology 26, 32-46.

Anderson, M.J. (2006). Distance-Based Tests for Homogeneity of Multivariate Dispersions. Biometrics 62, 245-253.

Anderson, M.J., and Walsh, D.C.I. (2013). PERMANOVA, ANOSIM, and the Mantel test in the face of heterogeneous dispersions: What null hypothesis are you testing? Ecological Monographs $83,557-574$.

Andrews, S. (2019). "FastQC: a quality control tool for high throughput sequence data". 0.11.8 ed.

Banerji, S., Simon, S., Tille, A., Fruth, A., and Flieger, A. (2020). Genome-based Salmonella serotyping as the new gold standard. Sci Rep 10, 4333.

980

981

982

983

Bankevich, A., Nurk, S., Antipov, D., Gurevich, A.A., Dvorkin, M., Kulikov, A.S., Lesin, V.M., Nikolenko, S.I., Pham, S., Prjibelski, A.D., Pyshkin, A.V., Sirotkin, A.V., Vyahhi, N., Tesler, G., Alekseyev, M.A., and Pevzner, P.A. (2012). SPAdes: a new genome assembly algorithm and its applications to single-cell sequencing. J Comput Biol 19, 455-477.

Boerlin, P., Wissing, A., Aarestrup, F.M., Frey, J., and Nicolet, J. (2001). Antimicrobial growth promoter ban and resistance to macrolides and vancomycin in enterococci from pigs. J Clin Microbiol 39, 4193-4195.

Bolger, A.M., Lohse, M., and Usadel, B. (2014). Trimmomatic: a flexible trimmer for Illumina sequence data. Bioinformatics 30, 2114-2120.

Boore, A.L., Hoekstra, R.M., Iwamoto, M., Fields, P.I., Bishop, R.D., and Swerdlow, D.L. (2015). Salmonella enterica Infections in the United States and Assessment of Coefficients of Variation: A Novel Approach to Identify Epidemiologic Characteristics of Individual Serotypes, 1996-2011. PLoS One 10, e0145416.

Bouckaert, R. (2014). Correcting for constant sites in BEAST2 [Online]. Available: https://groups.google.com/forum/\#!topic/beast-users/QfBHMOqImFE [Accessed September 9, 2020].

Bouckaert, R., Heled, J., Kuhnert, D., Vaughan, T., Wu, C.H., Xie, D., Suchard, M.A., Rambaut, A., and Drummond, A.J. (2014). BEAST 2: a software platform for Bayesian evolutionary analysis. PLoS Comput Biol 10, e1003537.

Bouckaert, R., Vaughan, T.G., Barido-Sottani, J., Duchene, S., Fourment, M., Gavryushkina, A., Heled, J., Jones, G., Kuhnert, D., De Maio, N., Matschiner, M., Mendes, F.K., Muller, N.F., Ogilvie, H.A., Du Plessis, L., Popinga, A., Rambaut, A., Rasmussen, D., Siveroni, I., Suchard, M.A., Wu, C.H., Xie, D., Zhang, C., Stadler, T., and Drummond, A.J. (2019). 
BEAST 2.5: An advanced software platform for Bayesian evolutionary analysis. PLoS Comput Biol 15, e1006650.

1006 Camacho, C., Coulouris, G., Avagyan, V., Ma, N., Papadopoulos, J., Bealer, K., and Madden, T.L.

Canton, R., Gonzalez-Alba, J.M., and Galán, J.C. (2012). CTX-M Enzymes: Origin and Diffusion. Frontiers in Microbiology 3.

1010 Carattoli, A., Zankari, E., Garcia-Fernandez, A., Voldby Larsen, M., Lund, O., Villa, L., Moller Aarestrup, F., and Hasman, H. (2014). In silico detection and typing of plasmids using PlasmidFinder and plasmid multilocus sequence typing. Antimicrob Agents Chemother 58, 3895-3903.

Carroll, L.M., Gaballa, A., Guldimann, C., Sullivan, G., Henderson, L.O., and Wiedmann, M. (2019). Identification of Novel Mobilized Colistin Resistance Gene mcr-9 in a Multidrug-Resistant, Colistin-Susceptible Salmonella enterica Serotype Typhimurium Isolate. mBio 10.

Carroll, L.M., Huisman, J.S., and Wiedmann, M. (2020a). Twentieth-century emergence of antimicrobial resistant human- and bovine-associated Salmonella enterica serotype Typhimurium lineages in New York State. Sci Rep 10, 14428.

Carroll, L.M., Kovac, J., Miller, R.A., and Wiedmann, M. (2017a). Rapid, High-Throughput Identification of Anthrax-Causing and Emetic Bacillus cereus Group Genome Assemblies via BTyper, a Computational Tool for Virulence-Based Classification of Bacillus cereus Group Isolates by Using Nucleotide Sequencing Data. Appl Environ Microbiol 83.

Carroll, L.M., Wiedmann, M., Den Bakker, H., Siler, J., Warchocki, S., Kent, D., Lyalina, S., Davis, M., Sischo, W., Besser, T., Warnick, L.D., and Pereira, R.V. (2017b). Whole-Genome Sequencing of Drug-Resistant Salmonella enterica Isolates from Dairy Cattle and Humans in New York and Washington States Reveals Source and Geographic Associations. Appl Environ Microbiol 83.

Carroll, L.M., Wiedmann, M., and Kovac, J. (2020b). Proposal of a Taxonomic Nomenclature for the Bacillus cereus Group Which Reconciles Genomic Definitions of Bacterial Species with Clinical and Industrial Phenotypes. mBio 11, e00034-00020.

Centers for Disease Control and Prevention (2019). Antibiotic Resistance Threats in the United States, 2019. Atlanta, GA: U.S. Department of Health and Human Services, CDC.

Centers for Disease Control and Prevention (2020). Serotypes and the Importance of Serotyping Salmonella [Online]. CDC. Available:

https://www.cdc.gov/salmonella/reportspubs/salmonella-atlas/serotyping-importance.html [Accessed 25 May 2021 2021].

Chang, W., Cheng, J., Allaire, J., Xie, Y., and Mcpherson, J. (2020). "shiny: Web Application Framework for R. R package version 1.4.0.2. https://CRAN.R-project.org/package=shiny".

Chen, T., and Guestrin, C. (2016). "XGBoost: A Scalable Tree Boosting System", in: Proceedings of the 22nd ACM SIGKDD International Conference on Knowledge Discovery and Data Mining. (San Francisco, California, USA: Association for Computing Machinery).

Chiu, C.H., Su, L.H., and Chu, C. (2004). Salmonella enterica serotype Choleraesuis: epidemiology, pathogenesis, clinical disease, and treatment. Clin Microbiol Rev 17, 311-322. 
1045

1046

1047

1048

1049

1050

1051

1052

1053

1054

1055

1056

1057

1058

1059

1060

1061

1062

1063

1064

1065

1066

1067

1068

1069

1070

1071

1072

1073

1074

1075

1076

1077

1078

1079

1080

1081

1082

1083

1084

1085

1086

1087

Cingolani, P., Platts, A., Wang Le, L., Coon, M., Nguyen, T., Wang, L., Land, S.J., Lu, X., and Ruden, D.M. (2012). A program for annotating and predicting the effects of single nucleotide polymorphisms, SnpEff: SNPs in the genome of Drosophila melanogaster strain w1118; iso2; iso-3. Fly (Austin) 6, 80-92.

Clarke, K.R. (1993). Non-parametric multivariate analyses of changes in community structure. Australian Journal of Ecology 18, 117-143.

Clarke, L., Pelin, A., Phan, M., and Wong, A. (2020). The effect of environmental heterogeneity on the fitness of antibiotic resistance mutations in Escherichia coli. Evolutionary Ecology 34, 379-390.

Clausen, P.T., Zankari, E., Aarestrup, F.M., and Lund, O. (2016). Benchmarking of methods for identification of antimicrobial resistance genes in bacterial whole genome data. $J$ Antimicrob Chemother 71, 2484-2488.

Cleary, J.G., Braithwaite, R., Gaastra, K., Hilbush, B.S., Inglis, S., Irvine, S.A., Jackson, A., Littin, R., Rathod, M., Ware, D., Zook, J.M., Trigg, L., and De La Vega, F.M. (2015). Comparing Variant Call Files for Performance Benchmarking of Next-Generation Sequencing Variant Calling Pipelines. bioRxiv, 023754.

Cooper, A.L., Low, A.J., Koziol, A.G., Thomas, M.C., Leclair, D., Tamber, S., Wong, A., Blais, B.W., and Carrillo, C.D. (2020). Systematic Evaluation of Whole Genome Sequence-Based Predictions of Salmonella Serotype and Antimicrobial Resistance. Front Microbiol 11, 549.

Croucher, N.J., Page, A.J., Connor, T.R., Delaney, A.J., Keane, J.A., Bentley, S.D., Parkhill, J., and Harris, S.R. (2015). Rapid phylogenetic analysis of large samples of recombinant bacterial whole genome sequences using Gubbins. Nucleic Acids Res 43, e15.

Cummings, K.J., Warnick, L.D., Davis, M.A., Eckmann, K., Grohn, Y.T., Hoelzer, K., Macdonald, K., Root, T.P., Siler, J.D., Mcguire, S.M., Wiedmann, M., Wright, E.M., Zansky, S.M., and Besser, T.E. (2012). Farm animal contact as risk factor for transmission of bovine-associated Salmonella subtypes. Emerg Infect Dis 18, 1929-1936.

Cummings, K.J., Warnick, L.D., Elton, M., Grohn, Y.T., Mcdonough, P.L., and Siler, J.D. (2010a). The effect of clinical outbreaks of salmonellosis on the prevalence of fecal Salmonella shedding among dairy cattle in New York. Foodborne Pathog Dis 7, 815-823.

Cummings, K.J., Warnick, L.D., Elton, M., Rodriguez-Rivera, L.D., Siler, J.D., Wright, E.M., Grohn, Y.T., and Wiedmann, M. (2010b). Salmonella enterica serotype Cerro among dairy cattle in New York: an emerging pathogen? Foodborne Pathog Dis 7, 659-665.

Davidson, K.E., Byrne, B.A., Pires, A.F.A., Magdesian, K.G., and Pereira, R.V. (2018). Antimicrobial resistance trends in fecal Salmonella isolates from northern California dairy cattle admitted to a veterinary teaching hospital, 2002-2016. PLoS One 13, e0199928.

Delgado-Suarez, E.J., Selem-Mojica, N., Ortiz-Lopez, R., Gebreyes, W.A., Allard, M.W., BaronaGomez, F., and Rubio-Lozano, M.S. (2018). Whole genome sequencing reveals widespread distribution of typhoidal toxin genes and VirB/D4 plasmids in bovine-associated nontyphoidal Salmonella. Sci Rep 8, 9864.

Delsol, A.A., Anjum, M., Woodward, M.J., Sunderland, J., and Roe, J.M. (2003). The effect of chlortetracycline treatment and its subsequent withdrawal on multi-resistant Salmonella enterica serovar Typhimurium DT104 and commensal Escherichia coli in the pig. J Appl Microbiol 95, 1226-1234. 
1088

1089

1090

1091

1092

1093

1094

1095

1096

1097

1098

1099

1100

1101

1102

1103

1104

1105

1106

1107

1108

1109

1110

1111

1112

1113

1114

1115

1116

1117

1118

1119

1120

1121

1122

1123

1124

1125

1126

1127

1128

Diep, B., Barretto, C., Portmann, A.C., Fournier, C., Karczmarek, A., Voets, G., Li, S., Deng, X., and Klijn, A. (2019). Salmonella Serotyping; Comparison of the Traditional Method to a Microarray-Based Method and an in silico Platform Using Whole Genome Sequencing Data. Front Microbiol 10, 2554.

Drummond, A.J., Ho, S.Y., Phillips, M.J., and Rambaut, A. (2006). Relaxed phylogenetics and dating with confidence. PLoS Biol 4, e88.

Drummond, A.J., Rambaut, A., Shapiro, B., and Pybus, O.G. (2005). Bayesian coalescent inference of past population dynamics from molecular sequences. Mol Biol Evol 22, 1185-1192.

Dutil, L., Irwin, R., Finley, R., Ng, L.K., Avery, B., Boerlin, P., Bourgault, A.M., Cole, L., Daignault, D., Desruisseau, A., Demczuk, W., Hoang, L., Horsman, G.B., Ismail, J., Jamieson, F., Maki, A., Pacagnella, A., and Pillai, D.R. (2010). Ceftiofur resistance in Salmonella enterica serovar Heidelberg from chicken meat and humans, Canada. Emerg Infect Dis 16, 48-54.

Evans, B.A., and Amyes, S.G.B. (2014). OXA $\beta$-Lactamases. Clinical Microbiology Reviews 27, 241-263.

Ewels, P., Magnusson, M., Lundin, S., and Kaller, M. (2016). MultiQC: summarize analysis results for multiple tools and samples in a single report. Bioinformatics 32, 3047-3048.

Feldgarden, M., Brover, V., Haft, D.H., Prasad, A.B., Slotta, D.J., Tolstoy, I., Tyson, G.H., Zhao, S., Hsu, C.H., Mcdermott, P.F., Tadesse, D.A., Morales, C., Simmons, M., Tillman, G., Wasilenko, J., Folster, J.P., and Klimke, W. (2019). Validating the AMRFinder Tool and Resistance Gene Database by Using Antimicrobial Resistance Genotype-Phenotype Correlations in a Collection of Isolates. Antimicrob Agents Chemother 63.

Fenske, G.J., Thachil, A., Mcdonough, P.L., Glaser, A., and Scaria, J. (2019). Geography Shapes the Population Genomics of Salmonella enterica Dublin. Genome Biol Evol 11, 2220-2231.

Frech, G., Kehrenberg, C., and Schwarz, S. (2003). Resistance phenotypes and genotypes of multiresistant Salmonella enterica subsp. enterica serovar Typhimurium var. Copenhagen isolates from animal sources. J Antimicrob Chemother 51, 180-182.

Gardner, S.N., and Hall, B.G. (2013). When whole-genome alignments just won't work: kSNP v2 software for alignment-free SNP discovery and phylogenetics of hundreds of microbial genomes. PLoS One 8, e81760.

Gardner, S.N., Slezak, T., and Hall, B.G. (2015). kSNP3.0: SNP detection and phylogenetic analysis of genomes without genome alignment or reference genome. Bioinformatics 31, 2877-2878.

Garrison, E., and Marth, G. (2012). Haplotype-based variant detection from short-read sequencing. arXiv, 1207.3907.

Gorski, L., Parker, C.T., Liang, A., Cooley, M.B., Jay-Russell, M.T., Gordus, A.G., Atwill, E.R., and Mandrell, R.E. (2011). Prevalence, distribution, and diversity of Salmonella enterica in a major produce region of California. Appl Environ Microbiol 77, 2734-2748.

Gupta, A., Fontana, J., Crowe, C., Bolstorff, B., Stout, A., Duyne, S.V., Hoekstra, M.P., Whichard, J.M., Barrett, T.J., and Group, T.N.a.R.M.S.P.W. (2003). Emergence of Multidrug-Resistant Salmonella enterica Serotype Newport Infections Resistant to Expanded-Spectrum Cephalosporins in the United States. The Journal of Infectious Diseases 188, 1707-1716.

This is a provisional file, not the final typeset article 
1129

1130

1131

1132

1133

1134

1135

1136

1137

1138

1139

1140

1141

1142

1143

1144

1145

1146

1147

1148

1149

1150

1151

1152

1153

1154

1155

1156

1157

1158

1159

1160

1161

1162

1163

1164

1165

1166

1167

1168

1169

Gupta, S.K., Padmanabhan, B.R., Diene, S.M., Lopez-Rojas, R., Kempf, M., Landraud, L., and Rolain, J.M. (2014). ARG-ANNOT, a new bioinformatic tool to discover antibiotic resistance genes in bacterial genomes. Antimicrob Agents Chemother 58, 212-220.

Gurevich, A., Saveliev, V., Vyahhi, N., and Tesler, G. (2013). QUAST: quality assessment tool for genome assemblies. Bioinformatics 29, 1072-1075.

Gutema, F.D., Agga, G.E., Abdi, R.D., De Zutter, L., Duchateau, L., and Gabriel, S. (2019). Prevalence and Serotype Diversity of Salmonella in Apparently Healthy Cattle: Systematic Review and Meta-Analysis of Published Studies, 2000-2017. Front Vet Sci 6, 102.

Harvey, R.R., Friedman, C.R., Crim, S.M., Judd, M., Barrett, K.A., Tolar, B., Folster, J.P., Griffin, P.M., and Brown, A.C. (2017). Epidemiology of Salmonella enterica Serotype Dublin Infections among Humans, United States, 1968-2013. Emerg Infect Dis 23.

Heled, J., and Bouckaert, R.R. (2013). Looking for trees in the forest: summary tree from posterior samples. BMC Evol Biol 13, 221.

Hendriksen, R.S., Bortolaia, V., Tate, H., Tyson, G.H., Aarestrup, F.M., and Mcdermott, P.F. (2019). Using Genomics to Track Global Antimicrobial Resistance. Front Public Health 7, 242.

Hendriksen, R.S., Pedersen, S.K., Leekitcharoenphon, P., Malorny, B., Borowiak, M., Battisti, A., Franco, A., Alba, P., Carfora, V., Ricci, A., Mastrorilli, E., Losasso, C., Longo, A., Petrin, S., Barco, L., Wołkowicz, T., Gierczyński, R., Zacharczuk, K., Wolaniuk, N., Wasyl, D., Zajac, M., Wieczorek, K., Półtorak, K., Petrovska-Holmes, L., Davies, R., Tang, Y., Grant, K., Underwood, A., Dallman, T., Painset, A., Hartman, H., Al-Shabib, A., and Cowley, L. (2018). Final report of ENGAGE - Establishing Next Generation sequencing Ability for Genomic analysis in Europe. EFSA Supporting Publications 15, 1431E.

Hoang, D.T., Chernomor, O., Von Haeseler, A., Minh, B.Q., and Vinh, L.S. (2018). UFBoot2: Improving the Ultrafast Bootstrap Approximation. Mol Biol Evol 35, 518-522.

Hoelzer, K., Moreno Switt, A.I., and Wiedmann, M. (2011). Animal contact as a source of human non-typhoidal salmonellosis. Vet Res 42, 34.

Hoelzer, K., Wong, N., Thomas, J., Talkington, K., Jungman, E., and Coukell, A. (2017).

Antimicrobial drug use in food-producing animals and associated human health risks: what, and how strong, is the evidence? BMC Veterinary Research 13, 211.

Holschbach, C.L., and Peek, S.F. (2018). Salmonella in Dairy Cattle. Vet Clin North Am Food Anim Pract 34, 133-154.

Hornish, R.E., and Kotarski, S.F. (2002). Cephalosporins in veterinary medicine - ceftiofur use in food animals. Curr Top Med Chem 2, 717-731.

Hunt, M., Mather, A.E., Sanchez-Buso, L., Page, A.J., Parkhill, J., Keane, J.A., and Harris, S.R. (2017). ARIBA: rapid antimicrobial resistance genotyping directly from sequencing reads. Microb Genom 3, e000131.

Ibrahim, G.M., and Morin, P.M. (2018). Salmonella Serotyping Using Whole Genome Sequencing. Front Microbiol 9, 2993.

Inouye, M., Dashnow, H., Raven, L.A., Schultz, M.B., Pope, B.J., Tomita, T., Zobel, J., and Holt, K.E. (2014). SRST2: Rapid genomic surveillance for public health and hospital microbiology labs. Genome Med 6, 90. 
1170

1171

1172

1173

1174

1175

1176

1177

1178

1179

1180

1181

1182

1183

1184

1185

1186

1187

1188

1189

1190

1191

1192

1193

1194

1195

1196

1197

1198

1199

1200

1201

1202

1203

1204

1205

1206

1207

1208

1209

1210

1211

Issenhuth-Jeanjean, S., Roggentin, P., Mikoleit, M., Guibourdenche, M., De Pinna, E., Nair, S., Fields, P.I., and Weill, F.X. (2014). Supplement 2008-2010 (no. 48) to the White-KauffmannLe Minor scheme. Res Microbiol 165, 526-530.

Jia, B., Raphenya, A.R., Alcock, B., Waglechner, N., Guo, P., Tsang, K.K., Lago, B.A., Dave, B.M., Pereira, S., Sharma, A.N., Doshi, S., Courtot, M., Lo, R., Williams, L.E., Frye, J.G., Elsayegh, T., Sardar, D., Westman, E.L., Pawlowski, A.C., Johnson, T.A., Brinkman, F.S., Wright, G.D., and Mcarthur, A.G. (2017). CARD 2017: expansion and model-centric curation of the comprehensive antibiotic resistance database. Nucleic Acids Res 45, D566-D573.

Jorgensen, J.H., and Ferraro, M.J. (2009). Antimicrobial susceptibility testing: a review of general principles and contemporary practices. Clin Infect Dis 49, 1749-1755.

Kalyaanamoorthy, S., Minh, B.Q., Wong, T.K.F., Von Haeseler, A., and Jermiin, L.S. (2017). ModelFinder: fast model selection for accurate phylogenetic estimates. Nat Methods 14, 587589.

Karon, A.E., Archer, J.R., Sotir, M.J., Monson, T.A., and Kazmierczak, J.J. (2007). Human multidrug-resistant Salmonella Newport infections, Wisconsin, 2003-2005. Emerg Infect Dis 13, 1777-1780.

Kass, R.E., and Raftery, A.E. (1995). Bayes Factors. Journal of the American Statistical Association 90, 773-795.

Kodama, Y., Shumway, M., Leinonen, R., and International Nucleotide Sequence Database, C. (2012). The Sequence Read Archive: explosive growth of sequencing data. Nucleic Acids Res 40, D54-56.

Kokot, M., Dlugosz, M., and Deorowicz, S. (2017). KMC 3: counting and manipulating k-mer statistics. Bioinformatics 33, 2759-2761.

Kruskal, J.B. (1964). Nonmetric multidimensional scaling: A numerical method. Psychometrika 29, 115-129.

Kuhn, M. (2008). Building Predictive Models in R Using the caret Package. 2008 28, 26.

Lakin, S.M., Dean, C., Noyes, N.R., Dettenwanger, A., Ross, A.S., Doster, E., Rovira, P., Abdo, Z., Jones, K.L., Ruiz, J., Belk, K.E., Morley, P.S., and Boucher, C. (2017). MEGARes: an antimicrobial resistance database for high throughput sequencing. Nucleic Acids Res 45, D574-D580.

Leekitcharoenphon, P., Hendriksen, R.S., Le Hello, S., Weill, F.X., Baggesen, D.L., Jun, S.R., Ussery, D.W., Lund, O., Crook, D.W., Wilson, D.J., and Aarestrup, F.M. (2016). Global Genomic Epidemiology of Salmonella enterica Serovar Typhimurium DT104. Appl Environ Microbiol 82, 2516-2526.

Leinonen, R., Sugawara, H., Shumway, M., and International Nucleotide Sequence Database, C. (2011). The sequence read archive. Nucleic Acids Res 39, D19-21.

Lewis, J.S., 2nd, Herrera, M., Wickes, B., Patterson, J.E., and Jorgensen, J.H. (2007). First report of the emergence of CTX-M-type extended-spectrum beta-lactamases (ESBLs) as the predominant ESBL isolated in a U.S. health care system. Antimicrob Agents Chemother 51, 4015-4021.

Lewis, P.O. (2001). A likelihood approach to estimating phylogeny from discrete morphological character data. Syst Biol 50, 913-925.

This is a provisional file, not the final typeset article 
1212

1213

1214

1215

1216

1217

1218

1219

1220

1221

1222

1223

1224

1225

1226

1227

1228

1229

1230

1231

1232

1233

1234

1235

1236

1237

1238

1239

1240

1241

1242

1243

1244

1245

1246

1247

1248

1249

1250

1251

1252

Li, H. (2011). A statistical framework for SNP calling, mutation discovery, association mapping and population genetical parameter estimation from sequencing data. Bioinformatics 27, 29872993.

Li, H. (2013). Aligning sequence reads, clone sequences and assembly contigs with BWA-MEM. arXiv, 1303.3997.

Li, H. (2018). Minimap2: pairwise alignment for nucleotide sequences. Bioinformatics 34, 30943100 .

Li, H. (2019). "Seqtk: a fast and lightweight tool for processing sequences in the FASTA or FASTQ format". 1.2-r102-dirty ed.).

Li, H., and Durbin, R. (2009). Fast and accurate short read alignment with Burrows-Wheeler transform. Bioinformatics 25, 1754-1760.

Li, H., Handsaker, B., Wysoker, A., Fennell, T., Ruan, J., Homer, N., Marth, G., Abecasis, G., Durbin, R., and Genome Project Data Processing, S. (2009). The Sequence Alignment/Map format and SAMtools. Bioinformatics 25, 2078-2079.

Liao, J., Orsi, R.H., Carroll, L.M., Kovac, J., Ou, H., Zhang, H., and Wiedmann, M. (2019). Serotype-specific evolutionary patterns of antimicrobial-resistant Salmonella enterica. BMC Evol Biol 19, 132.

Liao, J., Orsi, R.H., Carroll, L.M., and Wiedmann, M. (2020). Comparative genomics reveals different population structures associated with host and geographic origin in antimicrobialresistant Salmonella enterica. Environ Microbiol.

Liebana, E., Carattoli, A., Coque, T.M., Hasman, H., Magiorakos, A.P., Mevius, D., Peixe, L., Poirel, L., Schuepbach-Regula, G., Torneke, K., Torren-Edo, J., Torres, C., and Threlfall, J. (2013). Public health risks of enterobacterial isolates producing extended-spectrum beta-lactamases or AmpC beta-lactamases in food and food-producing animals: an EU perspective of epidemiology, analytical methods, risk factors, and control options. Clin Infect Dis 56, 10301037.

Llarena, A.K., Zhang, J., Vehkala, M., Valimaki, N., Hakkinen, M., Hanninen, M.L., Roasto, M., Maesaar, M., Taboada, E., Barker, D., Garofolo, G., Camma, C., Di Giannatale, E., Corander, J., and Rossi, M. (2016). Monomorphic genotypes within a generalist lineage of Campylobacter jejuni show signs of global dispersion. Microb Genom 2, e000088.

Luceron, C.O., Meixeira, A.P., Sanz, I.A., Deleyto, V.C., Leon, S.H., and Ruiz, L.G. (2017). Notes from the Field: An Outbreak of Salmonella Typhimurium Associated with Playground Sand in a Preschool Setting - Madrid, Spain, September-October 2016. MMWR Morb Mortal Wkly Rep 66, 256-257.

Maechler, M., Rousseeuw, P., Struyf, A., Hubert , M., and Hornik, K. (2019). "cluster: Cluster Analysis Basics and Extensions". 2.1.0 ed.).

Mather, A.E., Reid, S.W., Maskell, D.J., Parkhill, J., Fookes, M.C., Harris, S.R., Brown, D.J., Coia, J.E., Mulvey, M.R., Gilmour, M.W., Petrovska, L., De Pinna, E., Kuroda, M., Akiba, M., Izumiya, H., Connor, T.R., Suchard, M.A., Lemey, P., Mellor, D.J., Haydon, D.T., and Thomson, N.R. (2013). Distinguishable epidemics of multidrug-resistant Salmonella Typhimurium DT104 in different hosts. Science 341, 1514-1517. 
1253

1254

1255

1256

1257

1258

1259

1260

1261

1262

1263

1264

1265

1266

1267

1268

1269

1270

1271

1272

1273

1274

1275

1276

1277

1278

1279

1280

1281

1282

1283

1284

1285

1286

1287

1288

1289

1290

1291

1292

1293

1294

Mcdermott, P.F., Bodeis, S.M., English, L.L., White, D.G., Walker, R.D., Zhao, S., Simjee, S., and Wagner, D.D. (2002). Ciprofloxacin resistance in Campylobacter jejuni evolves rapidly in chickens treated with fluoroquinolones. J Infect Dis 185, 837-840.

Mcdermott, P.F., Tyson, G.H., Kabera, C., Chen, Y., Li, C., Folster, J.P., Ayers, S.L., Lam, C., Tate, H.P., and Zhao, S. (2016). Whole-Genome Sequencing for Detecting Antimicrobial Resistance in Nontyphoidal Salmonella. Antimicrobial Agents and Chemotherapy 60, 55155520.

Mellor, K.C., Petrovska, L., Thomson, N.R., Harris, K., Reid, S.W.J., and Mather, A.E. (2019). Antimicrobial Resistance Diversity Suggestive of Distinct Salmonella Typhimurium Sources or Selective Pressures in Food-Production Animals. Front Microbiol 10, 708.

Melnyk, A.H., Wong, A., and Kassen, R. (2015). The fitness costs of antibiotic resistance mutations. Evol Appl 8, 273-283.

Mentaberre, G., Porrero, M.C., Navarro-Gonzalez, N., Serrano, E., Dominguez, L., and Lavin, S. (2013). Cattle drive Salmonella infection in the wildlife-livestock interface. Zoonoses Public Health 60, 510-518.

Minh, B.Q., Nguyen, M.A., and Von Haeseler, A. (2013). Ultrafast approximation for phylogenetic bootstrap. Mol Biol Evol 30, 1188-1195.

Mohammed, M., Le Hello, S., Leekitcharoenphon, P., and Hendriksen, R. (2017). The invasome of Salmonella Dublin as revealed by whole genome sequencing. BMC Infect Dis 17, 544.

Moller, S., Du Plessis, L., and Stadler, T. (2018). Impact of the tree prior on estimating clock rates during epidemic outbreaks. Proc Natl Acad Sci U S A 115, 4200-4205.

Mughini-Gras, L., Enserink, R., Friesema, I., Heck, M., Van Duynhoven, Y., and Van Pelt, W. (2014). Risk factors for human salmonellosis originating from pigs, cattle, broiler chickens and egg laying hens: a combined case-control and source attribution analysis. PLoS One 9, e87933.

Nguyen, L.T., Schmidt, H.A., Von Haeseler, A., and Minh, B.Q. (2015). IQ-TREE: a fast and effective stochastic algorithm for estimating maximum-likelihood phylogenies. Mol Biol Evol $32,268-274$.

Nguyen, M., Long, S.W., Mcdermott, P.F., Olsen, R.J., Olson, R., Stevens, R.L., Tyson, G.H., Zhao, S., and Davis, J.J. (2019). Using Machine Learning To Predict Antimicrobial MICs and Associated Genomic Features for Nontyphoidal Salmonella. Journal of Clinical Microbiology 57, e01260-01218.

Oksanen, J., Blanchet, F.G., Friendly, M., Kindt, R., Legendre, P., Mcglinn, D., Minchin, P.R., O'hara, R.B., Simpson, G.L., Solymos, P., Stevens, M.H.H., Szoecs, E., and Wagner, H. (2019). "vegan: Community Ecology Package. R package version 2.5-6. https://CRAN.Rproject.org/package=vegan".).

Page, A.J., Cummins, C.A., Hunt, M., Wong, V.K., Reuter, S., Holden, M.T., Fookes, M., Falush, D., Keane, J.A., and Parkhill, J. (2015). Roary: rapid large-scale prokaryote pan genome analysis. Bioinformatics 31, 3691-3693.

Page, A.J., Taylor, B., Delaney, A.J., Soares, J., Seemann, T., Keane, J.A., and Harris, S.R. (2016). SNP-sites: rapid efficient extraction of SNPs from multi-FASTA alignments. Microb Genom 2, e000056.

This is a provisional file, not the final typeset article 
1295

1296

1297

1298

1299

1300

1301

1302

1303

1304

1305

1306

1307

1308

1309

1310

1311

1312

1313

1314

1315

1316

1317

1318

1319

1320

1321

1322

1323

1324

1325

1326

1327

1328

1329

1330

1331

1332

1333

1334

1335

Palma, F., Manfreda, G., Silva, M., Parisi, A., Barker, D.O.R., Taboada, E.N., Pasquali, F., and Rossi, M. (2018). Genome-wide identification of geographical segregated genetic markers in Salmonella enterica serovar Typhimurium variant 4,[5],12:i. Scientific Reports 8, 15251.

Paradis, E., Claude, J., and Strimmer, K. (2004). APE: Analyses of Phylogenetics and Evolution in R language. Bioinformatics 20, 289-290.

Paradis, E., and Schliep, K. (2019). ape 5.0: an environment for modern phylogenetics and evolutionary analyses in R. Bioinformatics 35, 526-528.

Parks, D.H., Imelfort, M., Skennerton, C.T., Hugenholtz, P., and Tyson, G.W. (2015). CheckM: assessing the quality of microbial genomes recovered from isolates, single cells, and metagenomes. Genome Res 25, 1043-1055.

Pereira, R., Williams, D.R., Rossitto, P., Adaska, J., Okello, E., Champagne, J., Lehenbauer, T.W., Li, X., Chase, J., Nguyen, T., Pires, A.F.A., Atwill, E.R., and Aly, S.S. (2019). Association between herd management practices and antimicrobial resistance in Salmonella spp. from cull dairy cattle in Central California. PeerJ 7, e6546.

Quinlan, A.R. (2014). BEDTools: The Swiss-Army Tool for Genome Feature Analysis. Curr Protoc Bioinformatics 47, 1112 11-34.

Quinlan, A.R., and Hall, I.M. (2010). BEDTools: a flexible suite of utilities for comparing genomic features. Bioinformatics 26, 841-842.

R Core Team (2019). "R: A Language and Environment for Statistical Computing". 3.6.1 ed. (Vienna, Austria: R Foundation for Statistical Computing).

R Hackathon (2019). "phylobase: Base Package for Phylogenetic Structures and Comparative Data". 0.8.6 ed.).

Rambaut, A., Drummond, A.J., Xie, D., Baele, G., and Suchard, M.A. (2018). Posterior Summarization in Bayesian Phylogenetics Using Tracer 1.7. Syst Biol 67, 901-904.

Rambaut, A., Lam, T.T., Max Carvalho, L., and Pybus, O.G. (2016). Exploring the temporal structure of heterochronous sequences using TempEst (formerly Path-O-Gen). Virus Evol 2, vew007.

Revell, L.J. (2012). phytools: an R package for phylogenetic comparative biology (and other things). Methods in Ecology and Evolution 3, 217-223.

Rodriguez, A., Pangloli, P., Richards, H.A., Mount, J.R., and Draughon, F.A. (2006). Prevalence of Salmonella in diverse environmental farm samples. J Food Prot 69, 2576-2580.

Rodriguez-Rivera, L.D., Wright, E.M., Siler, J.D., Elton, M., Cummings, K.J., Warnick, L.D., and Wiedmann, M. (2014). Subtype analysis of Salmonella isolated from subclinically infected dairy cattle and dairy farm environments reveals the presence of both human- and bovineassociated subtypes. Vet Microbiol 170, 307-316.

San Millan, A., and Maclean, R.C. (2017). Fitness Costs of Plasmids: a Limit to Plasmid Transmission. Microbiology Spectrum 5.

Scallan, E., Hoekstra, R.M., Angulo, F.J., Tauxe, R.V., Widdowson, M.A., Roy, S.L., Jones, J.L., and Griffin, P.M. (2011). Foodborne illness acquired in the United States--major pathogens. Emerg Infect Dis 17, 7-15.

Schliep, K.P. (2011). phangorn: phylogenetic analysis in R. Bioinformatics 27, 592-593. 
1336

1337

1338

1339

1340

1341

1342

1343

1344

1345

1346

1347

1348

1349

1350

1351

1352

1353

1354

1355

1356

1357

1358

1359

1360

1361

1362

1363

1364

1365

1366

1367

1368

1369

1370

1371

1372

1373

1374

1375

1376

1377

1378

Scott, A.M., Beller, E., Glasziou, P., Clark, J., Ranakusuma, R.W., Byambasuren, O., Bakhit, M., Page, S.W., Trott, D., and Mar, C.D. (2018). Is antimicrobial administration to food animals a direct threat to human health? A rapid systematic review. International Journal of Antimicrobial Agents 52, 316-323.

Seemann, T. (2014). Prokka: rapid prokaryotic genome annotation. Bioinformatics 30, 2068-2069.

Seemann, T. (2018). "ABRicate: Mass screening of contigs for antimicrobial resistance or virulence genes".

Seemann, T. (2019a). "samclip: Filter SAM file for soft and hard clipped alignments". 0.2 ed.

Seemann, T. (2019b). "Snippy: Rapid haploid variant calling and core genome alignment". 4.3.6 ed.

Skov, M.N., Madsen, J.J., Rahbek, C., Lodal, J., Jespersen, J.B., Jorgensen, J.C., Dietz, H.H., Chriel, M., and Baggesen, D.L. (2008). Transmission of Salmonella between wildlife and meatproduction animals in Denmark. J Appl Microbiol 105, 1558-1568.

Slowikowski, K. (2020). "ggrepel: Automatically Position Non-Overlapping Text Labels with 'ggplot2'. R package version 0.8.2. https://CRAN.R-project.org/package=ggrepel".

Soubrier, J., Steel, M., Lee, M.S.Y., Der Sarkissian, C., Guindon, S., Ho, S.Y.W., and Cooper, A. (2012). The Influence of Rate Heterogeneity among Sites on the Time Dependence of Molecular Rates. Molecular Biology and Evolution 29, 3345-3358.

Spika, J.S., Waterman, S.H., Hoo, G.W., St Louis, M.E., Pacer, R.E., James, S.M., Bissett, M.L., Mayer, L.W., Chiu, J.Y., Hall, B., and Et Al. (1987). Chloramphenicol-resistant Salmonella newport traced through hamburger to dairy farms. A major persisting source of human salmonellosis in California. N Engl J Med 316, 565-570.

Strachan, N.J.C., Rotariu, O., Lopes, B., Macrae, M., Fairley, S., Laing, C., Gannon, V., Allison, L.J., Hanson, M.F., Dallman, T., Ashton, P., Franz, E., Van Hoek, A.H.a.M., French, N.P., George, T., Biggs, P.J., and Forbes, K.J. (2015). Whole Genome Sequencing demonstrates that Geographic Variation of Escherichia coli O157 Genotypes Dominates Host Association. Scientific Reports 5, 14145.

Tack, B., Phoba, M.F., Barbe, B., Kalonji, L.M., Hardy, L., Van Puyvelde, S., Ingelbeen, B., Falay, D., Ngonda, D., Van Der Sande, M.a.B., Deborggraeve, S., Jacobs, J., and Lunguya, O. (2020). Non-typhoidal Salmonella bloodstream infections in Kisantu, DR Congo: Emergence of O5-negative Salmonella Typhimurium and extensive drug resistance. PLoS Negl Trop Dis 14, e0008121.

Tan, A., Abecasis, G.R., and Kang, H.M. (2015). Unified representation of genetic variants. Bioinformatics 31, 2202-2204.

Tang, K.L., Caffrey, N.P., Nobrega, D.B., Cork, S.C., Ronksley, P.E., Barkema, H.W., Polachek, A.J., Ganshorn, H., Sharma, N., Kellner, J.D., and Ghali, W.A. (2017). Restricting the use of antibiotics in food-producing animals and its associations with antibiotic resistance in foodproducing animals and human beings: a systematic review and meta-analysis. Lancet Planet Health 1, e316-e327.

Tassinari, E., Duffy, G., Bawn, M., Burgess, C.M., Mccabe, E.M., Lawlor, P.G., Gardiner, G., and Kingsley, R.A. (2019). Microevolution of antimicrobial resistance and biofilm formation of Salmonella Typhimurium during persistence on pig farms. Sci Rep 9, 8832.

Taylor, D.N., Bied, J.M., Munro, J.S., and Feldman, R.A. (1982). Salmonella dublin infections in the United States, 1979-1980. J Infect Dis 146, 322-327.

This is a provisional file, not the final typeset article 
1379

1380

1381

1382

1383

1384

1385

1386

1387

1388

1389

1390

1391

1392

1393

1394

1395

1396

1397

1398

1399

1400

1401

1402

1403

1404

1405

1406

1407

1408

1409

1410

1411

1412

1413

1414

1415

1416

1417

1418

1419

1420

The, H.C., Thanh, D.P., Holt, K.E., Thomson, N.R., and Baker, S. (2016). The genomic signatures of Shigella evolution, adaptation and geographical spread. Nat Rev Microbiol 14, 235-250.

Toth, J.D., Aceto, H.W., Rankin, S.C., and Dou, Z. (2011). Survival characteristics of Salmonella enterica serovar Newport in the dairy farm environment. J Dairy Sci 94, 5238-5246.

Tragesser, L.A., Wittum, T.E., Funk, J.A., Winokur, P.L., and Rajala-Schultz, P.J. (2006). Association between ceftiofur use and isolation of Escherichia coli with reduced susceptibility to ceftriaxone from fecal samples of dairy cows. Am J Vet Res 67, 1696-1700.

Uelze, L., Borowiak, M., Deneke, C., Szabo, I., Fischer, J., Tausch, S.H., and Malorny, B. (2020). Performance and Accuracy of Four Open-Source Tools for In Silico Serotyping of Salmonella spp. Based on Whole-Genome Short-Read Sequencing Data. Appl Environ Microbiol 86.

Uzzau, S., Brown, D.J., Wallis, T., Rubino, S., Leori, G., Bernard, S., Casadesus, J., Platt, D.J., and Olsen, J.E. (2000). Host adapted serotypes of Salmonella enterica. Epidemiol Infect 125, 229255.

Wang, C., Feng, Y., Liu, L., Wei, L., Kang, M., and Zong, Z. (2020). Identification of novel mobile colistin resistance gene mcr-10. Emerg Microbes Infect 9, 508-516.

Wang, X., Wang, Y., Zhou, Y., Li, J., Yin, W., Wang, S., Zhang, S., Shen, J., Shen, Z., and Wang, Y. (2018). Emergence of a novel mobile colistin resistance gene, $m c r-8$, in NDM-producing Klebsiella pneumoniae. Emerg Microbes Infect 7, 122.

Wickham, H. (2007). Reshaping Data with the reshape Package. 2007 21, 20.

Wickham, H. (2011). The Split-Apply-Combine Strategy for Data Analysis. 2011 40, 29.

Wickham, H. (2016). ggplot2: Elegant Graphics for Data Analysis. Springer-Verlag New York.

Wickham, H., François, R., Henry, L., and Müller, K. (2020). "dplyr: A Grammar of Data Manipulation". 0.8.5 ed.).

Wiethoelter, A.K., Beltran-Alcrudo, D., Kock, R., and Mor, S.M. (2015). Global trends in infectious diseases at the wildlife-livestock interface. Proc Natl Acad Sci U S A 112, 9662-9667.

Wong, V.K., Baker, S., Pickard, D.J., Parkhill, J., Page, A.J., Feasey, N.A., Kingsley, R.A., Thomson, N.R., Keane, J.A., Weill, F.X., Edwards, D.J., Hawkey, J., Harris, S.R., Mather, A.E., Cain, A.K., Hadfield, J., Hart, P.J., Thieu, N.T., Klemm, E.J., Glinos, D.A., Breiman, R.F., Watson, C.H., Kariuki, S., Gordon, M.A., Heyderman, R.S., Okoro, C., Jacobs, J., Lunguya, O., Edmunds, W.J., Msefula, C., Chabalgoity, J.A., Kama, M., Jenkins, K., Dutta, S., Marks, F., Campos, J., Thompson, C., Obaro, S., Maclennan, C.A., Dolecek, C., Keddy, K.H., Smith, A.M., Parry, C.M., Karkey, A., Mulholland, E.K., Campbell, J.I., Dongol, S., Basnyat, B., Dufour, M., Bandaranayake, D., Naseri, T.T., Singh, S.P., Hatta, M., Newton, P., Onsare, R.S., Isaia, L., Dance, D., Davong, V., Thwaites, G., Wijedoru, L., Crump, J.A., De Pinna, E., Nair, S., Nilles, E.J., Thanh, D.P., Turner, P., Soeng, S., Valcanis, M., Powling, J., Dimovski, K., Hogg, G., Farrar, J., Holt, K.E., and Dougan, G. (2015). Phylogeographical analysis of the dominant multidrug-resistant H58 clade of Salmonella Typhi identifies interand intracontinental transmission events. Nat Genet 47, 632-639.

World Health Organization (2014). Antimicrobial resistance: global report on surveillance. Geneva: World Health Organization (WHO).

World Health Organization (2017). Critically Important Antimicrobials for Human Medicine, 5th Revision. Geneva: World Health Organization. 
1421

1422

1423

1424

1425

1426

1427

1428

1429

1430

1431

1432

1433

1434

1435

1436

1437

1438

1439

Worley, J., Meng, J., Allard, M.W., Brown, E.W., and Timme, R.E. (2018). Salmonella enterica Phylogeny Based on Whole-Genome Sequencing Reveals Two New Clades and Novel Patterns of Horizontally Acquired Genetic Elements. MBio 9.

Xie, W., Lewis, P.O., Fan, Y., Kuo, L., and Chen, M.H. (2011). Improving marginal likelihood estimation for Bayesian phylogenetic model selection. Syst Biol 60, 150-160.

Yachison, C.A., Yoshida, C., Robertson, J., Nash, J.H.E., Kruczkiewicz, P., Taboada, E.N., Walker, M., Reimer, A., Christianson, S., Nichani, A., Pulsenet Canada Steering, C., and Nadon, C. (2017). The Validation and Implications of Using Whole Genome Sequencing as a Replacement for Traditional Serotyping for a National Salmonella Reference Laboratory. Front Microbiol 8, 1044.

Yang, W.-C., Chan, O.-W., Wu, T.-L., Chen, C.-L., Su, L.-H., and Chiu, C.-H. (2016). Development of ceftriaxone resistance in Salmonella enterica serotype Oranienburg during therapy for bacteremia. Journal of Microbiology, Immunology and Infection 49, 41-45.

Yang, Z. (1995). A space-time process model for the evolution of DNA sequences. Genetics 139, 993-1005.

Yoshida, C.E., Kruczkiewicz, P., Laing, C.R., Lingohr, E.J., Gannon, V.P., Nash, J.H., and Taboada, E.N. (2016). The Salmonella In Silico Typing Resource (SISTR): An Open Web-Accessible Tool for Rapidly Typing and Subtyping Draft Salmonella Genome Assemblies. PLoS One 11, e0147101.

Yu, G., Lam, T.T., Zhu, H., and Guan, Y. (2018). Two Methods for Mapping and Visualizing Associated Data on Phylogeny Using Ggtree. Mol Biol Evol 35, 3041-3043.

Yu, G., Smith, D.K., Zhu, H., Guan, Y., and Lam, T.T.-Y. (2017). ggtree: an r package for visualization and annotation of phylogenetic trees with their covariates and other associated data. Methods in Ecology and Evolution 8, 28-36.

Zankari, E., Hasman, H., Cosentino, S., Vestergaard, M., Rasmussen, S., Lund, O., Aarestrup, F.M., and Larsen, M.V. (2012). Identification of acquired antimicrobial resistance genes. $J$ Antimicrob Chemother 67, 2640-2644.

Zhang, S., Den Bakker, H.C., Li, S., Chen, J., Dinsmore, B.A., Lane, C., Lauer, A.C., Fields, P.I., and Deng, X. (2019). SeqSero2: Rapid and Improved Salmonella Serotype Determination Using Whole-Genome Sequencing Data. Appl Environ Microbiol 85.

Zwe, Y.H., Chin, S.F., Kohli, G.S., Aung, K.T., Yang, L., and Yuk, H.-G. (2020). Whole genome sequencing (WGS) fails to detect antimicrobial resistance (AMR) from heteroresistant subpopulation of Salmonella enterica. Food Microbiology 91, 103530.

This is a provisional file, not the final typeset article 
1457 Table 1. Statistics for the 15-antimicrobial phenotypic susceptibility/resistance prediction task for all antimicrobial resistance (AMR) 1458 determinant pipeline/database combinations. ${ }^{\text {a }}$

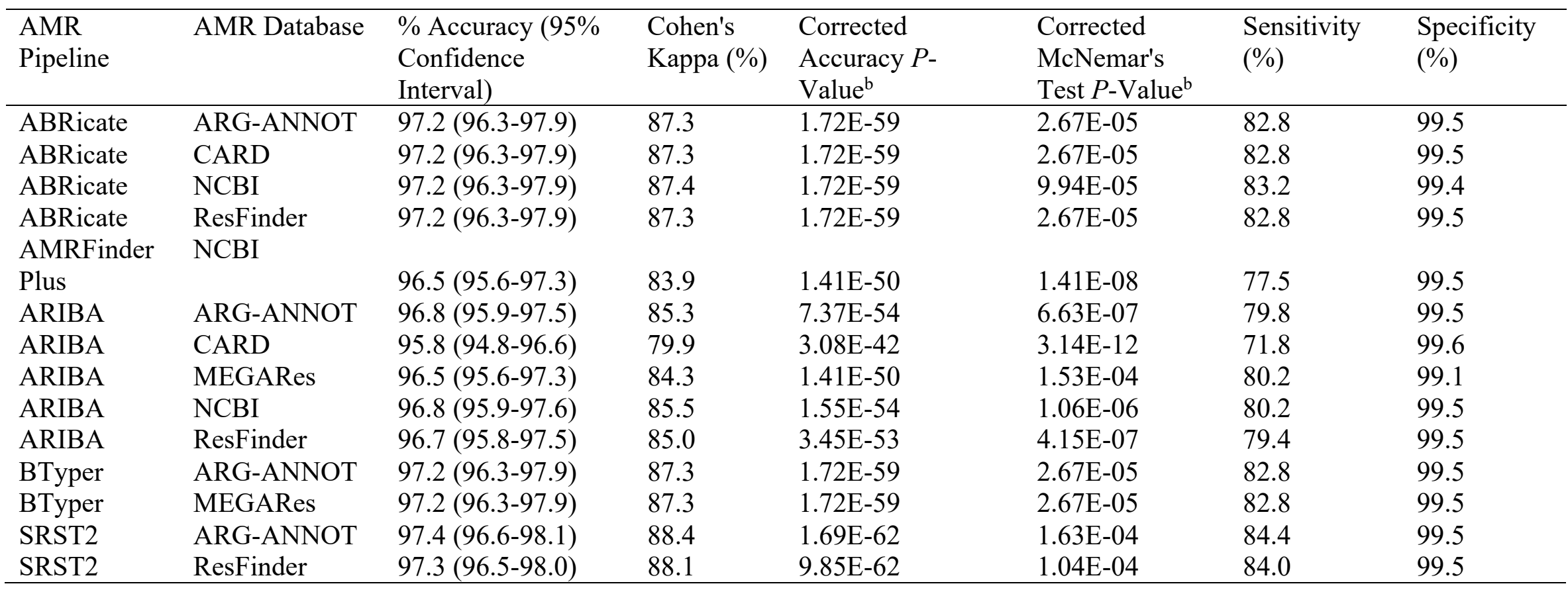

aStatistics were calculated using the confusionMatrix function in the caret package in R, with resistant ("R") phenotypes/genotypes treated as 1461 extended version of this table.

$1462{ }^{b}$ Adjusted using a Bonferroni correction. 
1464 Table 2. Results of PERMDISP2, ANOSIM, and PERMANOVA tests. ${ }^{\mathrm{a}}$

\begin{tabular}{lccl}
\hline Group & $\begin{array}{l}\text { PERMDISP2 Raw } \\
\boldsymbol{P} \text {-Value }(\boldsymbol{F})^{\mathbf{b}}\end{array}$ & $\begin{array}{l}\text { ANOSIM Raw } \boldsymbol{P} \text { - } \\
\text { Value }(\boldsymbol{R})^{\mathbf{c}}\end{array}$ & $\begin{array}{l}\text { PERMANOVA Raw } \boldsymbol{P} \text { - } \\
\text { Value }\left(\boldsymbol{R}^{2}\right)^{\mathbf{d}}\end{array}$ \\
\hline Pan-genome element presence/absence $(n=4,102)^{\mathrm{e}}$ & \\
Serotype & $2.0 \mathrm{E}-4(14.3)^{*}$ & $<1.0 \mathrm{E}-4(0.99)^{*}$ & $<1.0 \mathrm{E}-4(0.93)^{*}$ \\
Farm & $<1.0 \mathrm{E}-4(4.43)^{*}$ & $<1.0 \mathrm{E}-4(0.54)^{*}$ & $<1.0 \mathrm{E}-4(0.73)^{*}$ \\
Source & $0.071(3.40)$ & $0.99(-0.07)$ & $0.32(0.01)$ \\
Antimicrobial resistance and stress response gene presence/absence $(n=28)^{\mathrm{f}}$ \\
Serotype & $0.013(4.46)$ & $<1.0 \mathrm{E}-4(0.79)^{*}$ & $<1.0 \mathrm{E}-4(0.85)^{*}$ \\
Farm & $<1.0 \mathrm{E}-4(5.52)^{*}$ & $<1.0 \mathrm{E}-4(0.30)^{*}$ & $<1.0 \mathrm{E}-4(0.54)^{*}$ \\
Source & $0.74(0.01)$ & $0.79(-0.03)$ & $0.31(0.01)$ \\
\hline
\end{tabular}

$1465{ }^{a}$ All tests were performed using a Jaccard dissimilarity metric and 10,000 permutations; raw $P$-values 1466 are reported for all tests, with significant $P$-values $(P<0.05$ after a Bonferroni correction was applied 1467 to all values) denoted with an asterisk $(*)$.

1468 bANOVA-like permutation test applied to results obtained using the PERMDISP2 procedure for the 1469 analysis of multivariate homogeneity of group dispersions (i.e., variances), obtained using the 1470 betadisper and permutest functions in the vegan package in R; betadisper is a multivariate analogue 1471 of Levene's test for homogeneity of variances.

$1472{ }^{\mathrm{c} A n a l y s i s}$ of similarities (ANOSIM) test results obtained using the anosim function in the vegan 1473 package in $\mathrm{R}$

1474 dPermutational analysis of variance (PERMANOVA) test results obtained using the adonis2 function 1475 in the vegan package in $\mathrm{R}$

1476 Identified using Roary and a 70\% protein BLAST (BLASTP) identity threshold

1477 fDetected using AMRFinderPlus 
1480 Table 3. Summary of within-serotype evolutionary analyses. ${ }^{\text {a }}$

\begin{tabular}{|c|c|c|c|c|c|}
\hline Serotype & Isolates & $\begin{array}{l}\text { Core } \\
\text { SNPs } \\
\text { (Pairwise }^{\text {Pairwe) }} \\
\text { Range }\end{array}$ & $\begin{array}{l}\text { Clock/Population } \\
\text { Model }^{\mathbf{c}}\end{array}$ & $\begin{array}{l}\text { Mean/Median Tree } \\
\text { Height in Years } \\
(95 \% \text { HPD Interval) }\end{array}$ & $\begin{array}{l}\text { Mean/Median } \\
\text { Evolutionary Rate in } \\
\text { Substitutions/Site/Year } \\
\text { (95\% HPD Interval) }^{\mathrm{e}}\end{array}$ \\
\hline Anatum & 15 & $\begin{array}{l}337 \\
(0-257)\end{array}$ & Strict/Skyline & $\begin{array}{l}1484.9 / 1837.0 \\
(549.6-1980.1)\end{array}$ & $\begin{array}{l}1.67 \times 10^{-7} / 1.48 \times 10^{-7} \\
\left(6.92 \times 10^{-11}-3.86 \times 10^{-7}\right)\end{array}$ \\
\hline Cerro & 13 & $21(0-12)$ & Strict/Skyline & $\begin{array}{l}2008.2 / 2008.4 \\
(2007.6-2008.6)\end{array}$ & $\begin{array}{l}9.11 \times 10^{-7} / 8.94 \times 10^{-7} \\
\left(3.06 \times 10^{-7}-1.57 \times 10^{-6}\right)\end{array}$ \\
\hline Kentucky & 36 & $102(0-30)$ & Relaxed/Skyline & $\begin{array}{l}2004.1 / 2005.0 \\
(2000.8-2006.8)\end{array}$ & $\begin{array}{l}6.39 \times 10^{-7} / 6.34 \times 10^{-7} \\
\left(2.05 \times 10^{-7}-1.07 \times 10^{-6}\right)\end{array}$ \\
\hline Meleagridis & 19 & $27(0-9)$ & Strict/Skyline & $\begin{array}{l}2007.4 / 2007.5 \\
(2006.9-2007.7)\end{array}$ & $\begin{array}{l}6.88 \times 10^{-7} / 6.66 \times 10^{-7} \\
\left(2.90 \times 10^{-7}-1.12 \times 10^{-6}\right)\end{array}$ \\
\hline Newport & 16 & $52(0-38)$ & Relaxed/Skyline & $\begin{array}{l}2004.2 / 2004.6 \\
(2000.4-2007.8)\end{array}$ & $\begin{array}{l}9.02 \times 10^{-7} / 8.22 \times 10^{-7} \\
\left(2.64 \times 10^{-7}-1.65 \times 10^{-6}\right)\end{array}$ \\
\hline $\begin{array}{l}\text { Typhimurium } \\
\text { (Copenhagen) }\end{array}$ & 27 & $\begin{array}{l}732 \\
(0-634)\end{array}$ & Relaxed/Skyline & $\begin{array}{l}1936.0 / 1943.0 \\
(1864.7-1991.4)\end{array}$ & $\begin{array}{l}1.07 \times 10^{-6} / 9.66 \times 10^{-7} \\
\left(2.84 \times 10^{-7}-2.05 \times 10^{-6}\right)\end{array}$ \\
\hline
\end{tabular}

1481 a See Supplementary Table S5 for an extended version of this table; note that evolutionary rates may be higher than previously reported estimates for Salmonella populations isolated over a longer time frame, due to the small sample sizes and short temporal period characterized here (Moller et al., 2018).

$1484{ }^{b}$ Number of core SNPs identified among all genomes within the serotype after removing recombination with Gubbins; the 1485 range of pairwise SNP differences between isolates was calculated using the dist.gene function in the ape package in R.

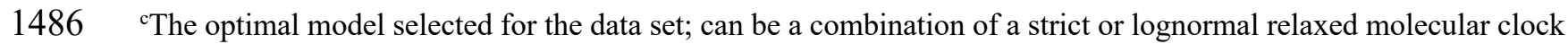
1487 ("Strict" or "Relaxed", respectively) and a Constant Coalescent or Coalescent Bayesian Skyline population model ("CC" 1488 or "Skyline", respectively); see Supplementary Table S5 for more details.

$1489{ }^{\mathrm{d}}$ The tree height parameter and its respective 95\% highest posterior density (HPD) interval reported by Tracer.

$1490{ }^{\mathrm{e}}$ Corresponds to the clockRate and rate.mean parameters estimated by BEAST2 for models using strict and lognormal 1491 relaxed molecular clock models, respectively, as reported by Tracer. 


\section{Figure Legends}

1496 Figure 1. Maximum likelihood phylogeny constructed using core SNPs identified among 570

1497 Salmonella isolate genomes. Publicly available genomes are denoted by black tip labels $(n=442)$,

1498 while genomes of bovine- and bovine farm-associated strains isolated in conjunction with this study

1499 are denoted by colored tip labels $(n=128)$. In cases where a discrepancy existed between the traditional serotype designation of an isolate and one or more in silico methods (i.e., SISTR and SeqSero2), the serotype assigned using two out of the three methods was selected as the final serotype to be used for phylogeny annotation. The phylogeny is rooted at the midpoint with branch lengths reported in substitutions per site. Core SNPs were identified among all genomes using kSNP3, while the phylogeny was constructed and annotated using IQ-TREE and bactaxR/ggtree, respectively.

Figure 2. Confusion matrices showcasing agreement between susceptible-intermediate-resistant (SIR) classification of 128 Salmonella isolates obtained using phenotypic resistance testing (denoted as the matrix "Reference") and five in silico methods (denoted as the matrix "Prediction") for 15 antimicrobials. Accuracy values above each matrix denote the percentage of correctly classified instances out of all instances. Kappa values above each matrix denote Cohen's kappa coefficient for the matrix, reported as a percent. For the phenotypic method, SIR classification was determined using NARMS breakpoints for Salmonella (accessed March 23, 2020). For the in silico antimicrobial resistance (AMR) determinant detection approaches, combinations of five pipelines and one to five AMR determinant databases were tested; isolate genomes that harbored one or more AMR determinants previously known to confer resistance to a particular antimicrobial were categorized as resistant to that antimicrobial ("R"), while those which did not were categorized as susceptible ("S"; see Supplementary Table S2 for all detected AMR determinants and their associated resistance classifications). For all AMR determinant detection methods, isolates that showed intermediate phenotypic resistance to an antimicrobial were categorized as susceptible ("S") rather than resistant, as this classification produced slightly better accuracy scores for all pipeline/database combinations. For AMR determinant detection methods that relied on nucleotide BLAST (i.e., ABRicate and BTyper), the confusion matrix obtained using the nucleotide identity and coverage threshold combination that produced the highest accuracy are shown (Supplementary Figure S3); for all other methods, confusion matrices obtained using default detection parameters are shown.

Figure 3. (A) Deviation of PATRIC3 predicted minimum inhibitory concentration (MIC) values from "true" MIC values obtained using phenotypic resistance testing for 14 antimicrobials (Y-axis), reported in number of dilution factors (X-axis). For each box plot, lower and upper box hinges correspond to the first and third quartiles, respectively. Lower and upper whiskers extend from the hinge to the smallest and largest values no more distant than 1.5 times the interquartile range from the hinge, respectively. Points represent pairwise distances that fall beyond the ends of the whiskers. Only isolates with raw MIC values available are included (126 of 128 isolates). (B) Confusion matrix showcasing agreement between susceptible-intermediate-resistant (SIR) classification of all 128 Salmonella isolates obtained using phenotypic resistance testing (denoted as the matrix "Reference") and PATRIC3 (denoted as the matrix "Prediction") for 14 antimicrobials. The accuracy value above the matrix denotes the percentage of correctly classified instances out of all instances. The Kappa value above the matrix denotes Cohen's kappa coefficient for the matrix, reported as a percent. For both the phenotypic and PATRIC3 methods, SIR classification was determined using NARMS

1538 breakpoints for Salmonella (accessed March 23, 2020). 
1540 Figure 4. Results of nonmetric multidimensional scaling (NMDS) performed using the presence and absence of (A) pan-genome elements ( $n=4,102$; identified using Roary), and (B) antimicrobial resistance $(\mathrm{AMR})$ and stress response genes $(n=28$; detected using AMRFinderPlus $)$ among 128 bovine-associated Salmonella isolates, plotted in two dimensions. Points represent isolates, while shaded regions and convex hulls correspond to isolate (1) serotypes (ANAT, Anatum; CERO, Cerro; KENT, Kentucky; MELA, Meleagridis; NEWP, Newport; TCOP, Typhimurium Copenhagen; TYPH, Typhimurium), (2) farm, and (3) source (BOV, bovine; ENV, bovine farm environment). For all plots, a Jaccard distance metric was used. For AMR/stress response genes (B), gene names/scores are plotted in dark gray text.

Figure 5. Rooted, time-scaled maximum clade credibility (MCC) phylogeny constructed using core SNPs identified among 15 Salmonella Anatum genomes isolated from subclinical bovine sources and the surrounding bovine farm environment. Tip label colors denote the ID of the farm from which each strain was isolated. Branch labels denote posterior probabilities of branch support. Time in years is plotted along the X-axis, and branch lengths are reported in years. The heatmap to the right of the phylogeny denotes (i) the susceptible-intermediate-resistant (SIR) classification of each isolate for each of 15 antimicrobials (obtained using phenotypic testing and NARMS breakpoints; orange); (ii) presence and absence of plasmid replicons (detected using ABRicate/PlasmidFinder and minimum nucleotide identity and coverage thresholds of 80 and 60\%, respectively; blue); (iii) presence and absence of antimicrobial resistance (AMR) and stress response genes (identified using AMRFinderPlus and default parameters; green). Core SNPs were identified using Snippy. The phylogeny was constructed using the results of ten independent runs using a strict clock model, the Standard_TVMef nucleotide substitution model, and the Coalescent Bayesian Skyline population model implemented in BEAST version 2.5.1, with 10\% burn-in applied to each run. LogCombiner-2 was used to combine BEAST $2 \log$ files, and TreeAnnotator-2 was used to construct the phylogeny using median node heights. Abbreviations for the 15 antimicrobials are: AMK, amikacin; AMC, amoxicillin-clavulanic acid; AMP, ampicillin; FOX, cefoxitin; TIO, ceftiofur; CRO, ceftriaxone; CHL, chloramphenicol; CIP, ciprofloxacin; GEN, gentamicin; KAN, kanamycin; NAL, nalidixic acid; STR, streptomycin; SXT, sulfamethoxazole-trimethoprim; SUL, sulfisoxazole; TET,

1568 tetracycline.

Figure 6. Rooted, time-scaled maximum clade credibility (MCC) phylogeny constructed using core SNPs identified among 13 Salmonella Cerro genomes isolated from subclinical bovine sources and the surrounding bovine farm environment. Tip label colors denote the ID of the farm from which each strain was isolated. Branch labels denote posterior probabilities of branch support. Time in years is plotted along the $\mathrm{X}$-axis, and branch lengths are reported in years. The heatmap to the right of the phylogeny denotes (i) the susceptible-intermediate-resistant (SIR) classification of each isolate for each of 15 antimicrobials (obtained using phenotypic testing and NARMS breakpoints; orange); (ii) presence of plasmid replicons (detected using ABRicate/PlasmidFinder and minimum nucleotide identity and coverage thresholds of 80 and $60 \%$, respectively; blue); (iii) presence of antimicrobial resistance (AMR) and stress response genes (identified using AMRFinderPlus and default parameters; green). Core SNPs were identified using Snippy. The phylogeny was constructed using the results of ten independent runs using a strict clock model, the Standard_TPM1 nucleotide substitution model, and the Coalescent Bayesian Skyline population model implemented in BEAST version 2.5.1, with 10\% burn-in applied to each run. LogCombiner-2 was used to combine BEAST 2 $\log$ files, and TreeAnnotator-2 was used to construct the phylogeny using common ancestor node heights. Abbreviations for the 15 antimicrobials are: AMK, amikacin; AMC, amoxicillin-clavulanic acid; AMP, ampicillin; FOX, cefoxitin; TIO, ceftiofur; CRO, ceftriaxone; CHL, chloramphenicol; 
1586 CIP, ciprofloxacin; GEN, gentamicin; KAN, kanamycin; NAL, nalidixic acid; STR, streptomycin; 1587 SXT, sulfamethoxazole-trimethoprim; SUL, sulfisoxazole; TET, tetracycline.

1588 Figure 7. Rooted, time-scaled maximum clade credibility (MCC) phylogeny constructed using core 1589 SNPs identified among 36 Salmonella Kentucky genomes isolated from subclinical bovine sources 1590 and the surrounding bovine farm environment. Tip label colors denote the ID of the farm from which 1591 each strain was isolated. Branch labels denote posterior probabilities of branch support. Time in years 1592 is plotted along the X-axis, and branch lengths are reported in years. The heatmap to the right of the 1593 phylogeny denotes (i) the susceptible-intermediate-resistant (SIR) classification of each isolate for each of 15 antimicrobials (obtained using phenotypic testing and NARMS breakpoints; orange); (ii) presence of plasmid replicons (detected using ABRicate/PlasmidFinder and minimum nucleotide identity and coverage thresholds of 80 and $60 \%$, respectively; blue); (iii) presence of antimicrobial resistance (AMR) and stress response genes (identified using AMRFinderPlus and default parameters; green). Core SNPs were identified using Snippy. The phylogeny was constructed using the results of ten independent runs using a relaxed lognormal clock model, the Standard_TVMef nucleotide substitution model, and the Coalescent Bayesian Skyline population model implemented in BEAST version 2.5.1, with 10\% burn-in applied to each run. LogCombiner-2 was used to combine BEAST 2 log files, and TreeAnnotator-2 was used to construct the phylogeny using common ancestor node heights. Abbreviations for the 15 antimicrobials are: AMK, amikacin; AMC, amoxicillin-clavulanic acid; AMP, ampicillin; FOX, cefoxitin; TIO, ceftiofur; CRO, ceftriaxone; CHL, chloramphenicol; CIP, ciprofloxacin; GEN, gentamicin; KAN, kanamycin; NAL, nalidixic acid; STR, streptomycin; SXT, sulfamethoxazole-trimethoprim; SUL, sulfisoxazole; TET, tetracycline.

1608 Figure 8. Rooted, time-scaled maximum clade credibility (MCC) phylogeny constructed using core 1609 SNPs identified among 19 Salmonella Meleagridis genomes isolated from subclinical bovine sources and the surrounding bovine farm environment. Tip label colors denote the ID of the farm from which each strain was isolated. Branch labels denote posterior probabilities of branch support. Time in years is plotted along the $\mathrm{X}$-axis, and branch lengths are reported in years. The heatmap to the right of the phylogeny denotes (i) the susceptible-intermediate-resistant (SIR) classification of each isolate for each of 15 antimicrobials (obtained using phenotypic testing and NARMS breakpoints; orange); (ii) presence and absence of plasmid replicons (detected using ABRicate/PlasmidFinder and minimum nucleotide identity and coverage thresholds of 80 and 60\%, respectively; blue); (iii) presence and absence of antimicrobial resistance (AMR) and stress response genes (identified using AMRFinderPlus and default parameters; green). Core SNPs were identified using Snippy. The phylogeny was constructed using the results of ten independent runs using a strict clock model, the Standard_TPM2 nucleotide substitution model, and the Coalescent Bayesian Skyline population model implemented in BEAST version 2.5.1, with 10\% burn-in applied to each run. LogCombiner-2 was used to combine BEAST 2 log files, and TreeAnnotator-2 was used to construct the phylogeny using common ancestor node heights. Abbreviations for the 15 antimicrobials are: AMK, amikacin; AMC, amoxicillin-clavulanic acid; AMP, ampicillin; FOX, cefoxitin; TIO, ceftiofur; CRO, ceftriaxone; CHL, chloramphenicol; CIP, ciprofloxacin; GEN, gentamicin; KAN, kanamycin; NAL, nalidixic acid; STR, streptomycin; SXT, sulfamethoxazole-trimethoprim; SUL, sulfisoxazole; TET, tetracycline.

1628 Figure 9. Rooted, time-scaled maximum clade credibility (MCC) phylogeny constructed using core 1629 SNPs identified among 16 Salmonella Newport genomes isolated from subclinical bovine sources 1630 and the surrounding bovine farm environment. Tip label colors denote the ID of the farm from which each strain was isolated. Branch labels denote posterior probabilities of branch support. Time in years 
1632 is plotted along the X-axis, and branch lengths are reported in years. The heatmap to the right of the 1633 phylogeny denotes (i) the susceptible-intermediate-resistant (SIR) classification of each isolate for 1634 each of 15 antimicrobials (obtained using phenotypic testing and NARMS breakpoints; orange); (ii) 1635 presence and absence of plasmid replicons (detected using ABRicate/PlasmidFinder and minimum 1636 nucleotide identity and coverage thresholds of 80 and $60 \%$, respectively; blue); (iii) presence and absence of antimicrobial resistance (AMR) and stress response genes (identified using AMRFinderPlus and default parameters; green). Core SNPs were identified using Snippy. The phylogeny was constructed using the results of ten independent runs using a relaxed lognormal clock model, the Standard_TPM1 nucleotide substitution model, and the Coalescent Bayesian Skyline population model implemented in BEAST version 2.5.1, with 10\% burn-in applied to each run. LogCombiner-2 was used to combine BEAST $2 \log$ files, and TreeAnnotator-2 was used to construct the phylogeny using common ancestor node heights. Abbreviations for the 15 antimicrobials are: AMK, amikacin; AMC, amoxicillin-clavulanic acid; AMP, ampicillin; FOX, cefoxitin; TIO, ceftiofur; CRO, ceftriaxone; CHL, chloramphenicol; CIP, ciprofloxacin; GEN, gentamicin; KAN, kanamycin; NAL, nalidixic acid; STR, streptomycin; SXT, sulfamethoxazole-trimethoprim; SUL, sulfisoxazole; TET, tetracycline.

1648 Figure 10. Rooted, time-scaled maximum clade credibility (MCC) phylogeny constructed using core SNPs identified among 27 Salmonella Typhimurium and Typhimurium Copenhagen genomes isolated from subclinical bovine sources and the surrounding bovine farm environment. Tip label colors denote the ID of the farm from which each strain was isolated. Circles to the left of tip labels denote isolates that were assigned to the Typhimurium Copenhagen variant of $S$. Typhimurium using SeqSero2 (teal) and/or traditional serotyping (pink). Branch labels denote posterior probabilities of branch support. Time in years is plotted along the X-axis, and branch lengths are reported in years. The heatmap to the right of the phylogeny denotes (i) the susceptible-intermediate-resistant (SIR) classification of each isolate for each of 15 antimicrobials (obtained using phenotypic testing and NARMS breakpoints; orange); (ii) presence and absence of plasmid replicons (detected using ABRicate/PlasmidFinder and minimum nucleotide identity and coverage thresholds of 80 and $60 \%$, respectively; blue); (iii) presence and absence of antimicrobial resistance (AMR) and stress response genes (identified using AMRFinderPlus and default parameters; green). Core SNPs were identified using Snippy. The phylogeny was constructed using the results of ten independent runs using a relaxed lognormal clock model, the Standard_TPM1 nucleotide substitution model, and the Coalescent Bayesian Skyline population model implemented in BEAST version 2.5.1, with 10\% burn-in applied to each run. LogCombiner-2 was used to combine BEAST 2 log files, and TreeAnnotator-2 was used to construct the phylogeny using common ancestor node heights. Abbreviations for the 15 antimicrobials are: AMK, amikacin; AMC, amoxicillin-clavulanic acid; AMP, ampicillin; FOX, cefoxitin; TIO, ceftiofur; CRO, ceftriaxone; CHL, chloramphenicol; CIP, ciprofloxacin; GEN, gentamicin; KAN, kanamycin; NAL, nalidixic acid; STR, streptomycin; SXT, sulfamethoxazole-trimethoprim; SUL, sulfisoxazole; TET, tetracycline. 
ARG-ANNOT

Accuracy $97 \%$ I Kappa $87 \%$

ABRicate
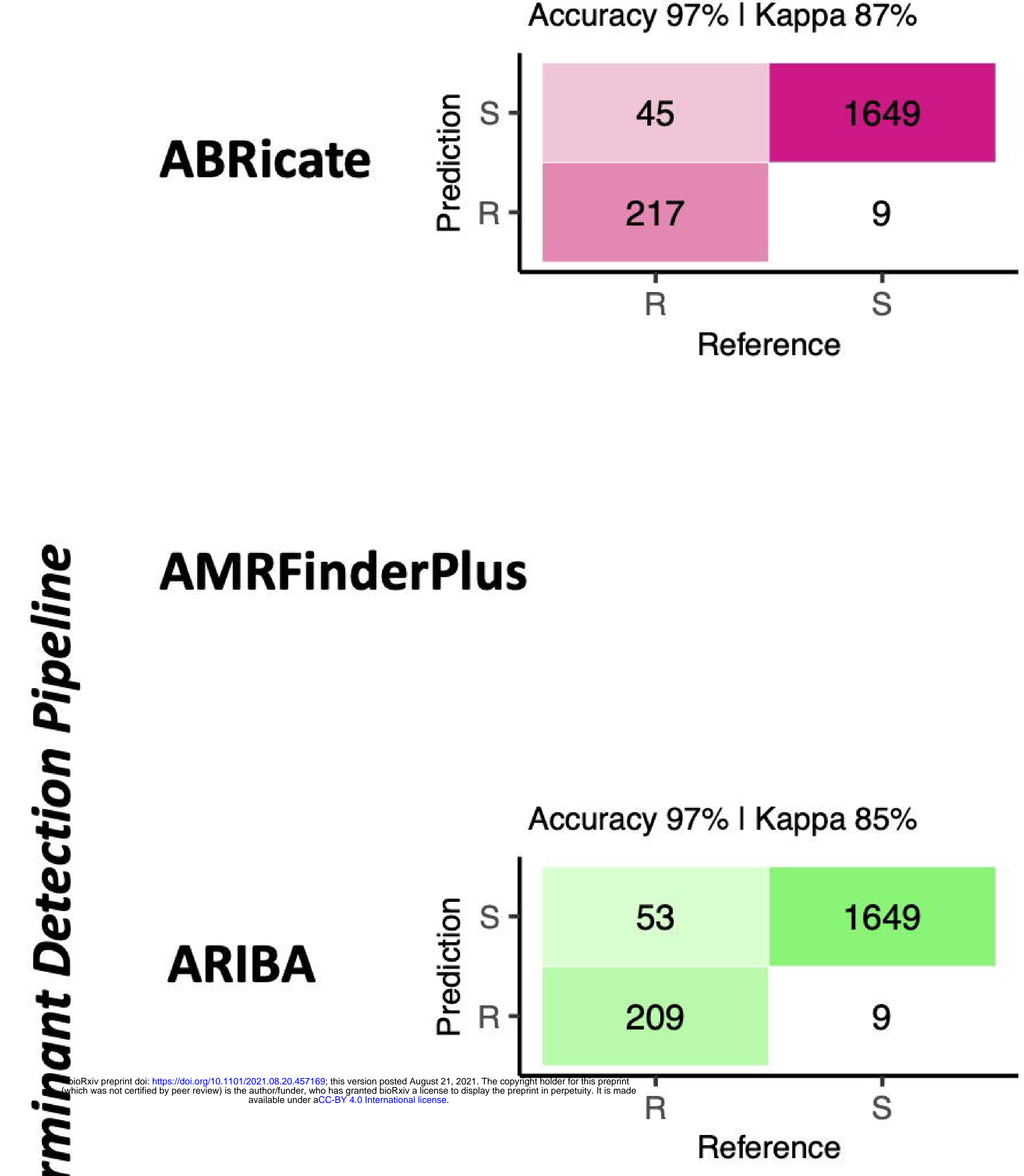

Accuracy $97 \%$ I Kappa $87 \%$

BTyper

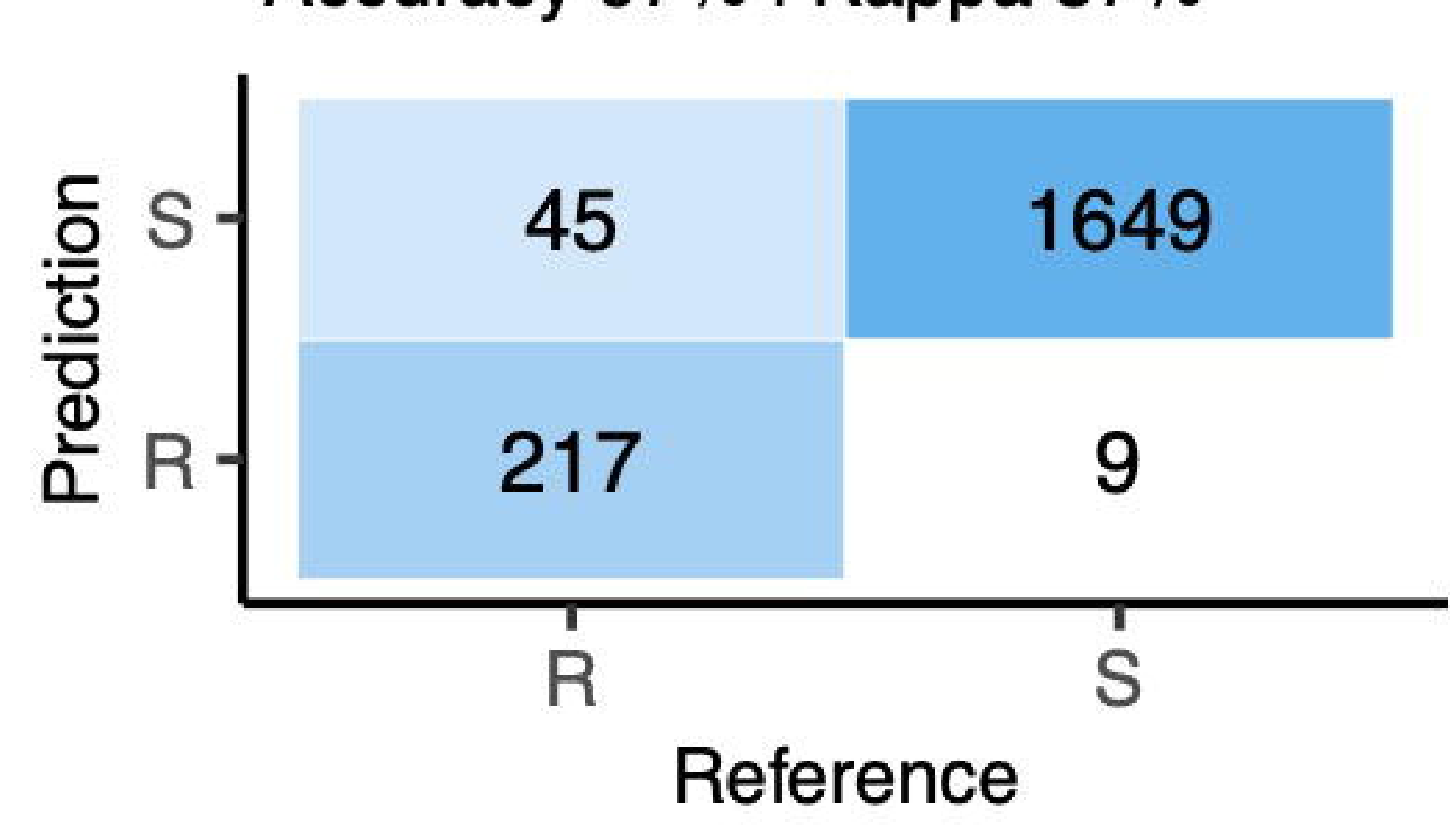

Accuracy $97 \%$ I Kappa $88 \%$

SRST2

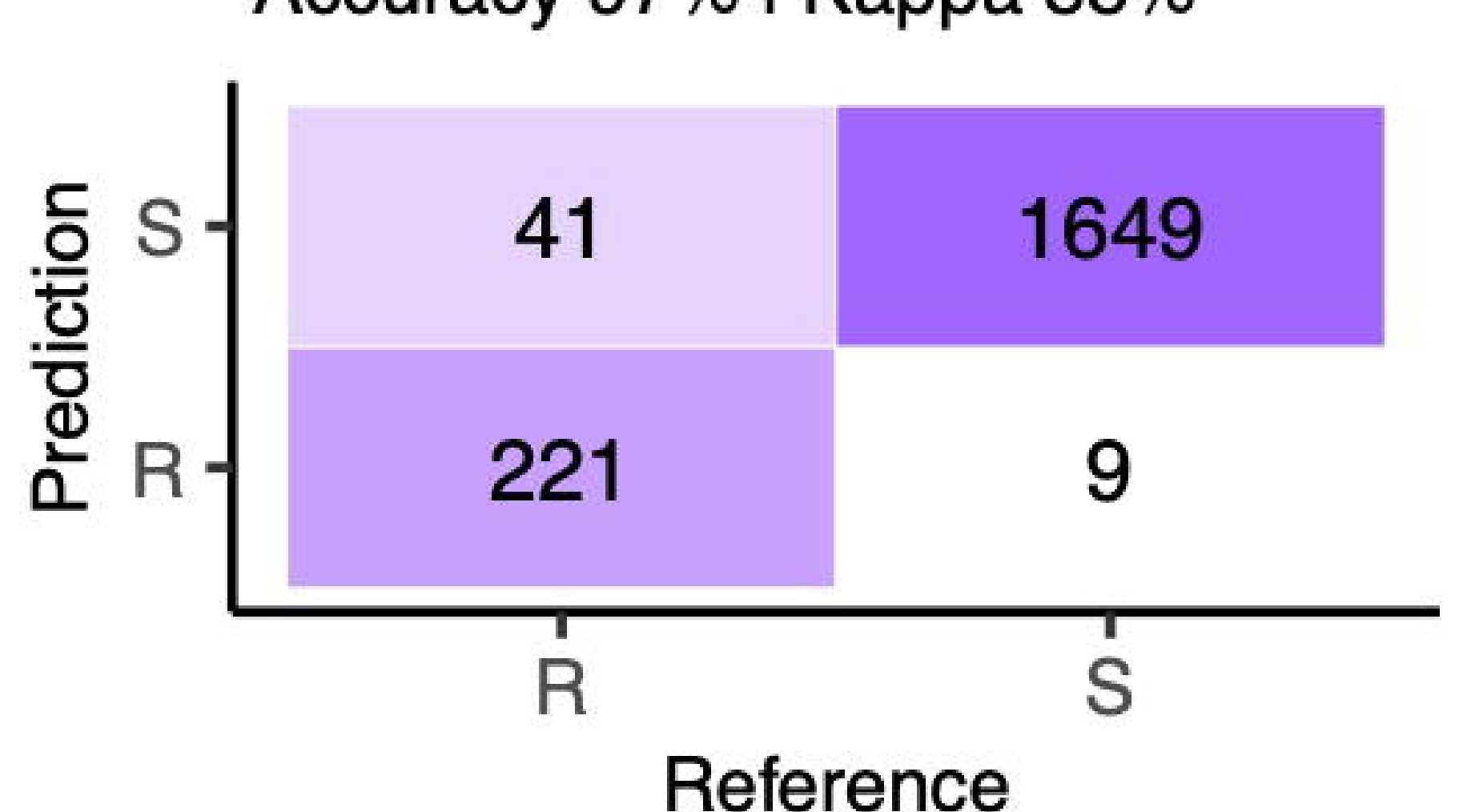

MEGARes

Accuracy $97 \%$ I Kappa $87 \%$

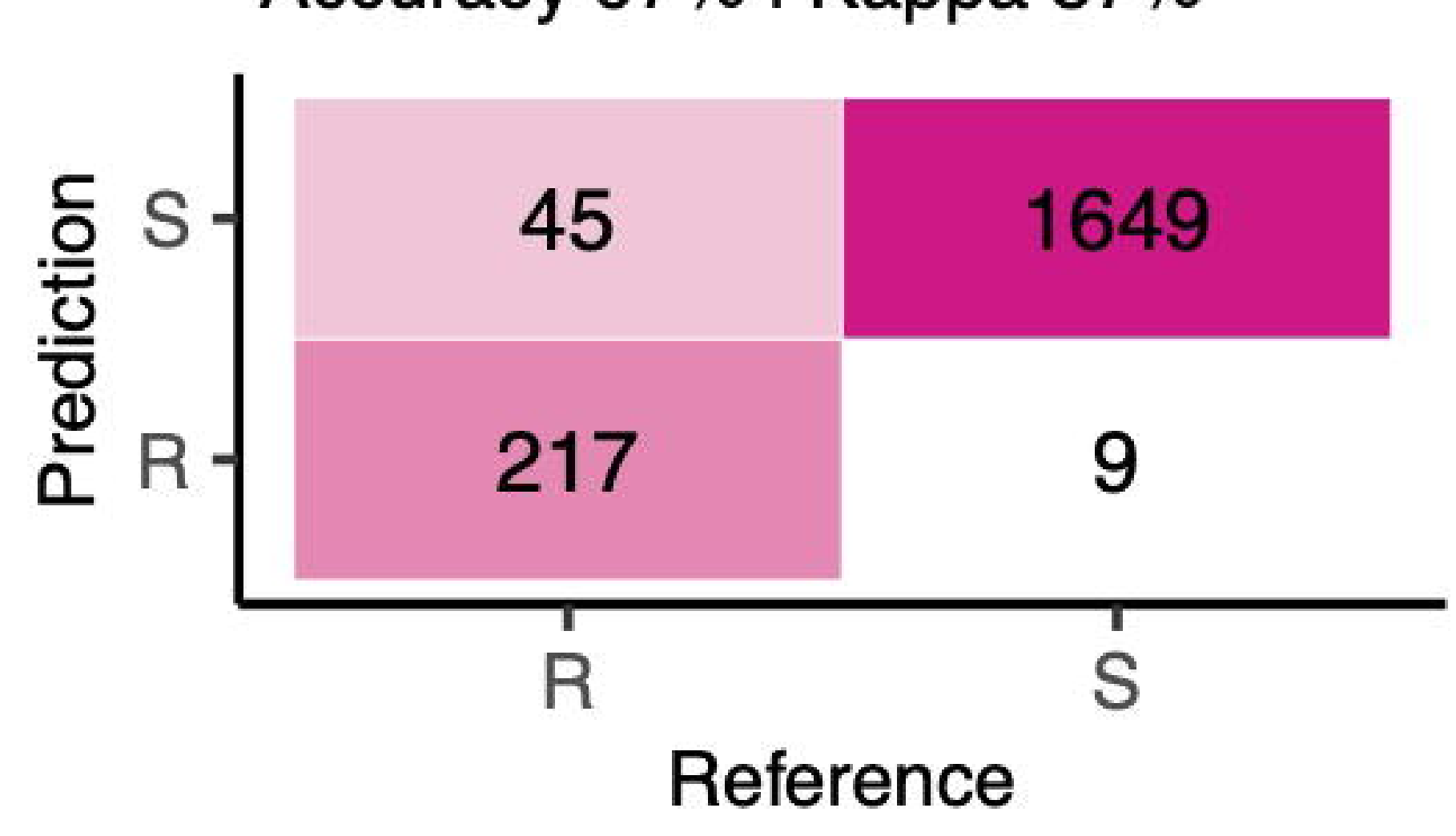

Accuracy 96\% I Kappa 80\%
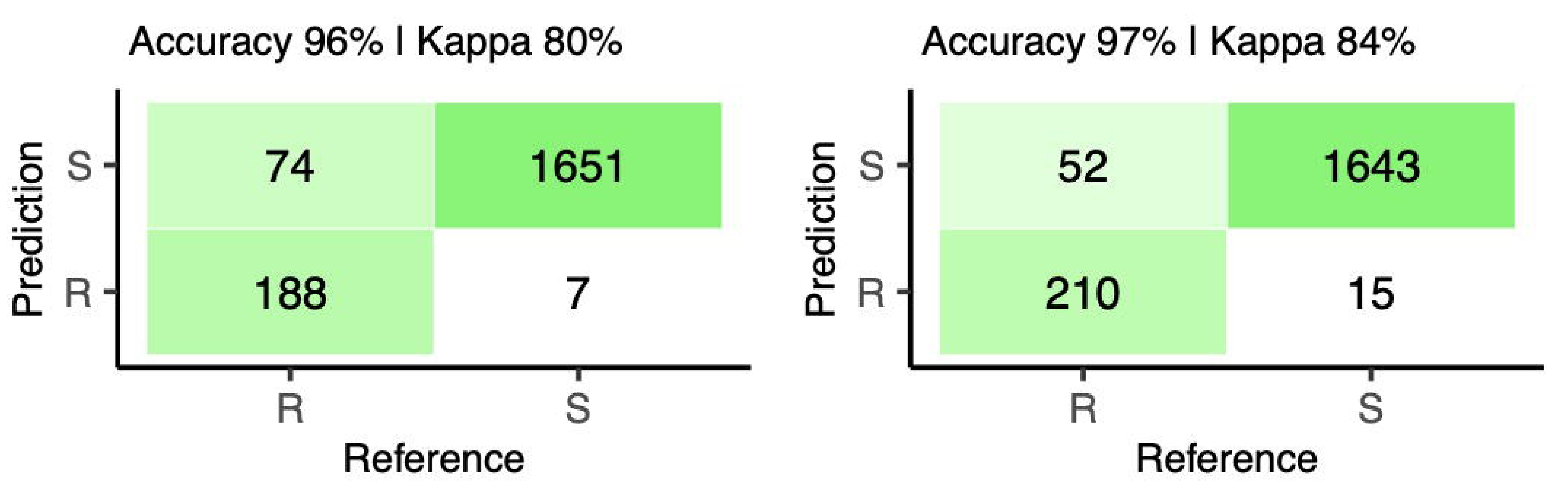

Accuracy $97 \%$ I Kappa $87 \%$

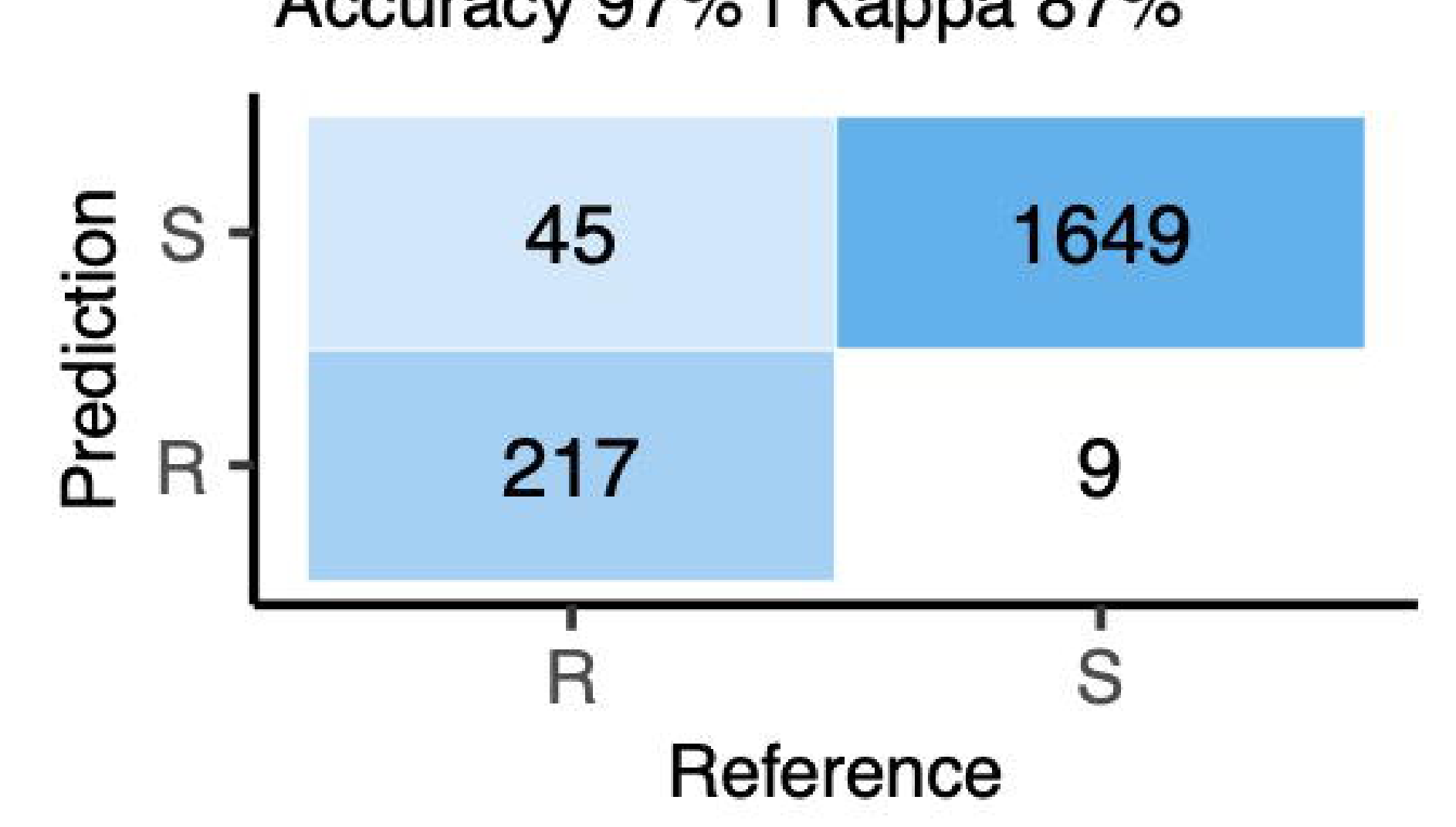

NCBI

Accuracy $97 \%$ I Kappa $87 \%$

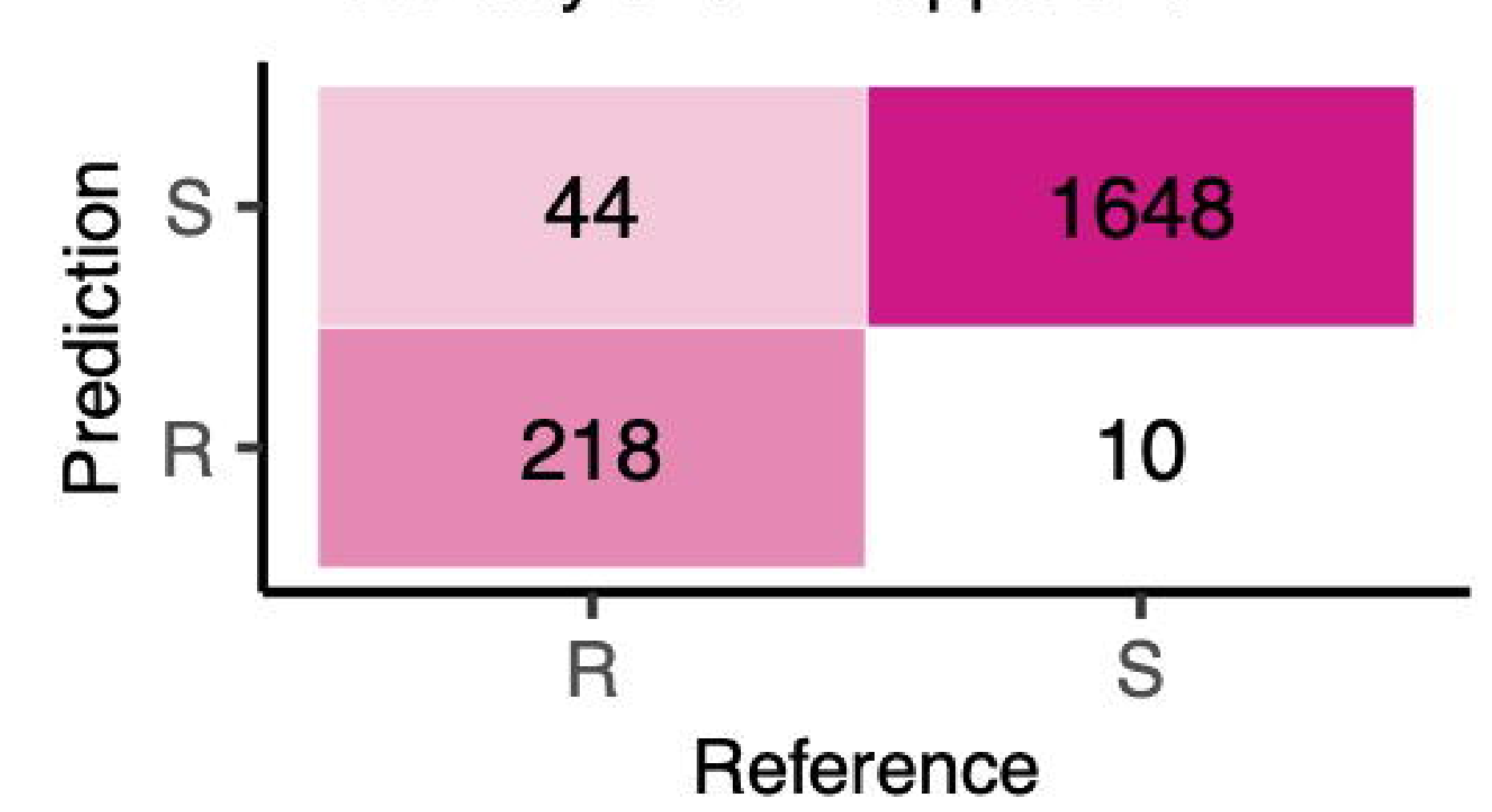

Accuracy $97 \%$ I Kappa $84 \%$

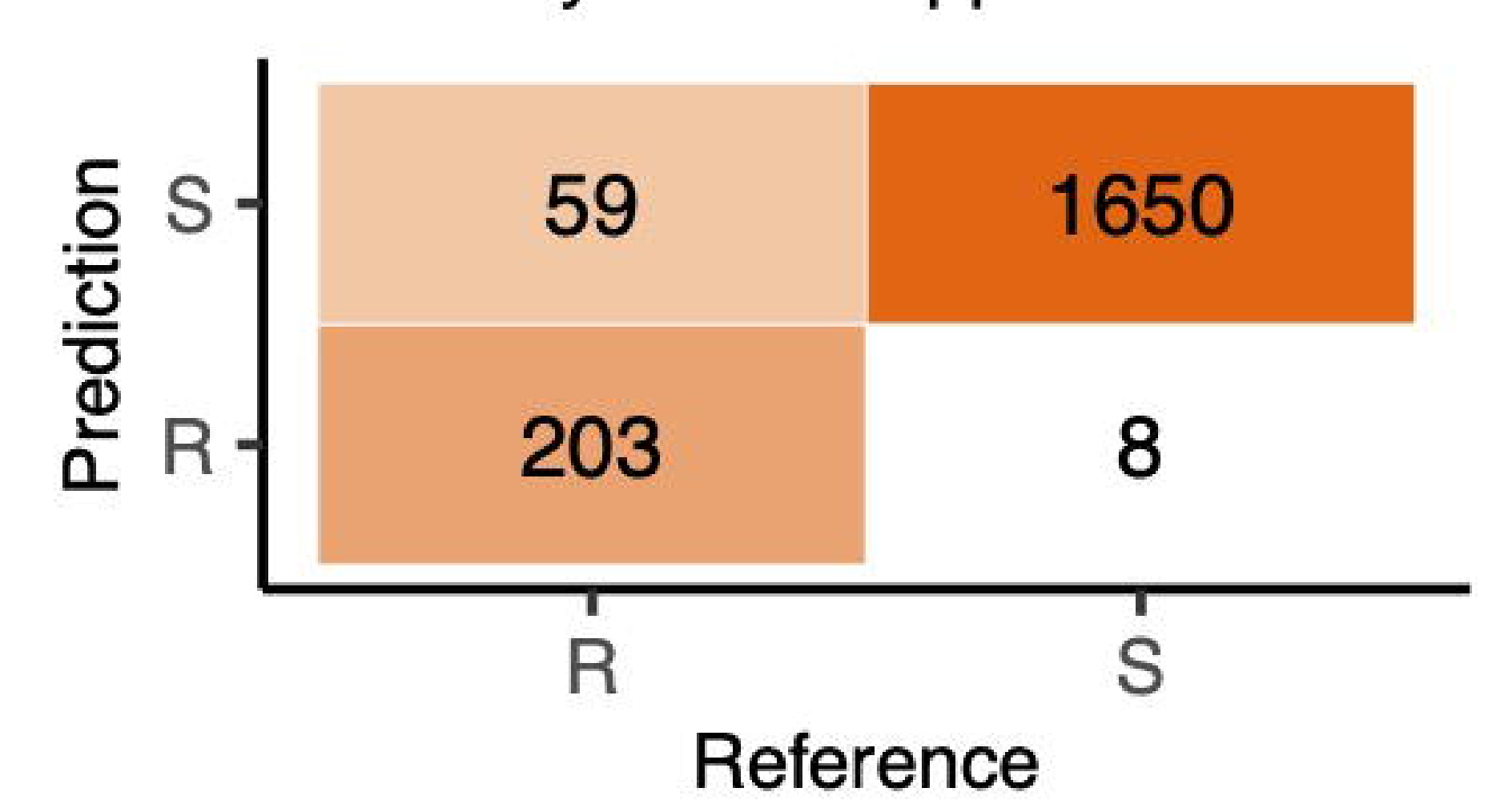

Accuracy $97 \%$ I Kappa $86 \%$
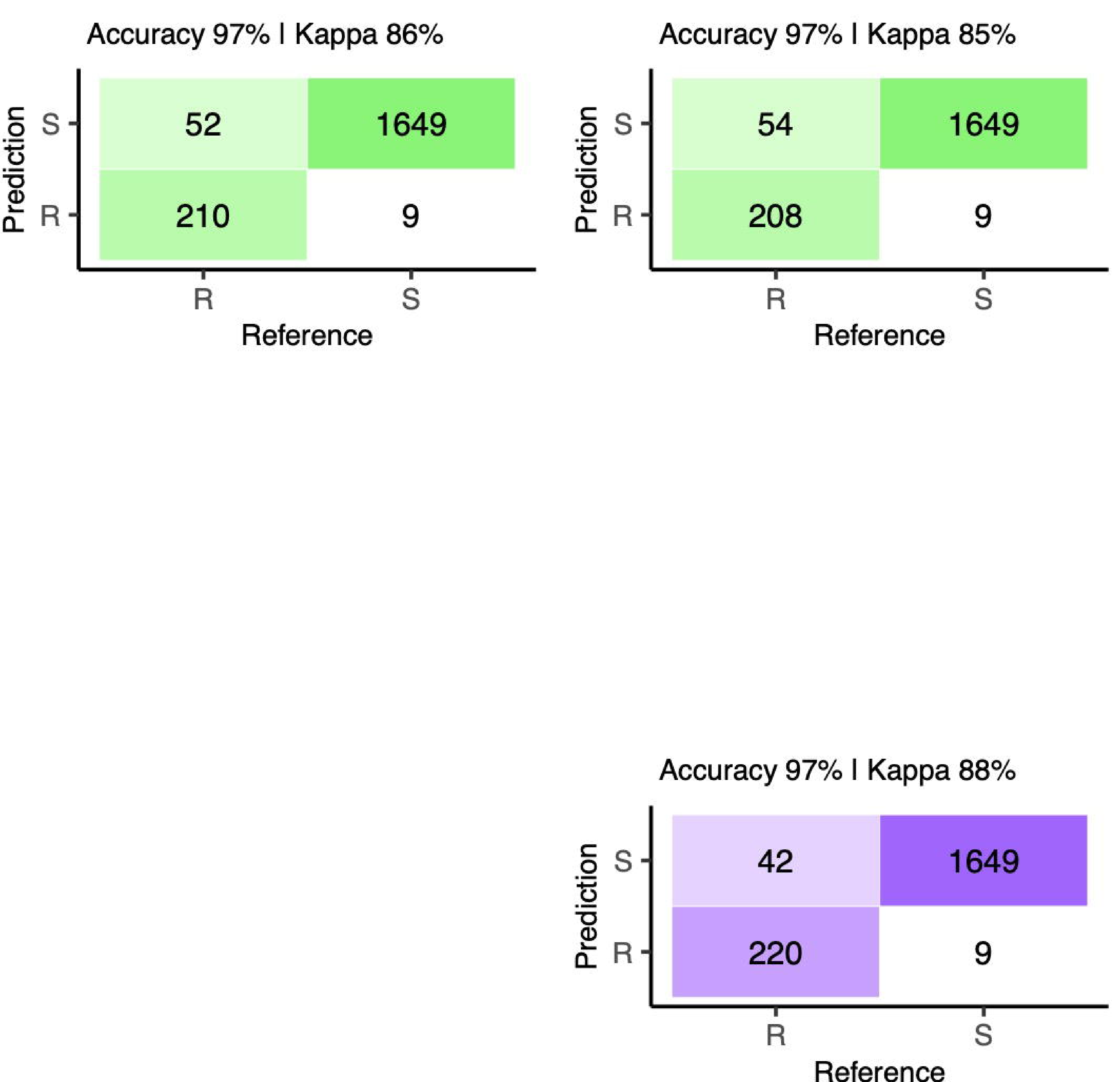

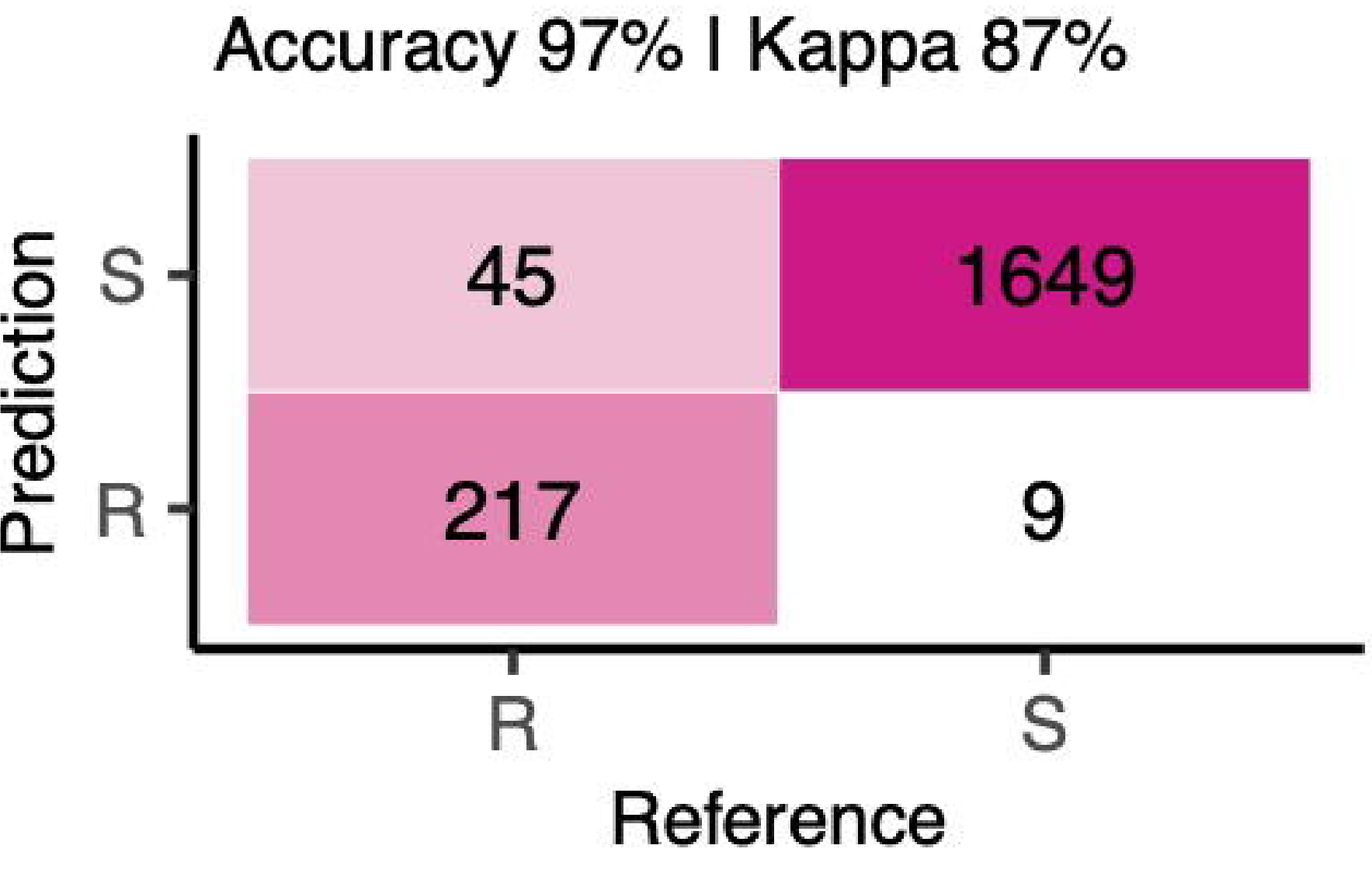

Reference
Resfinder 


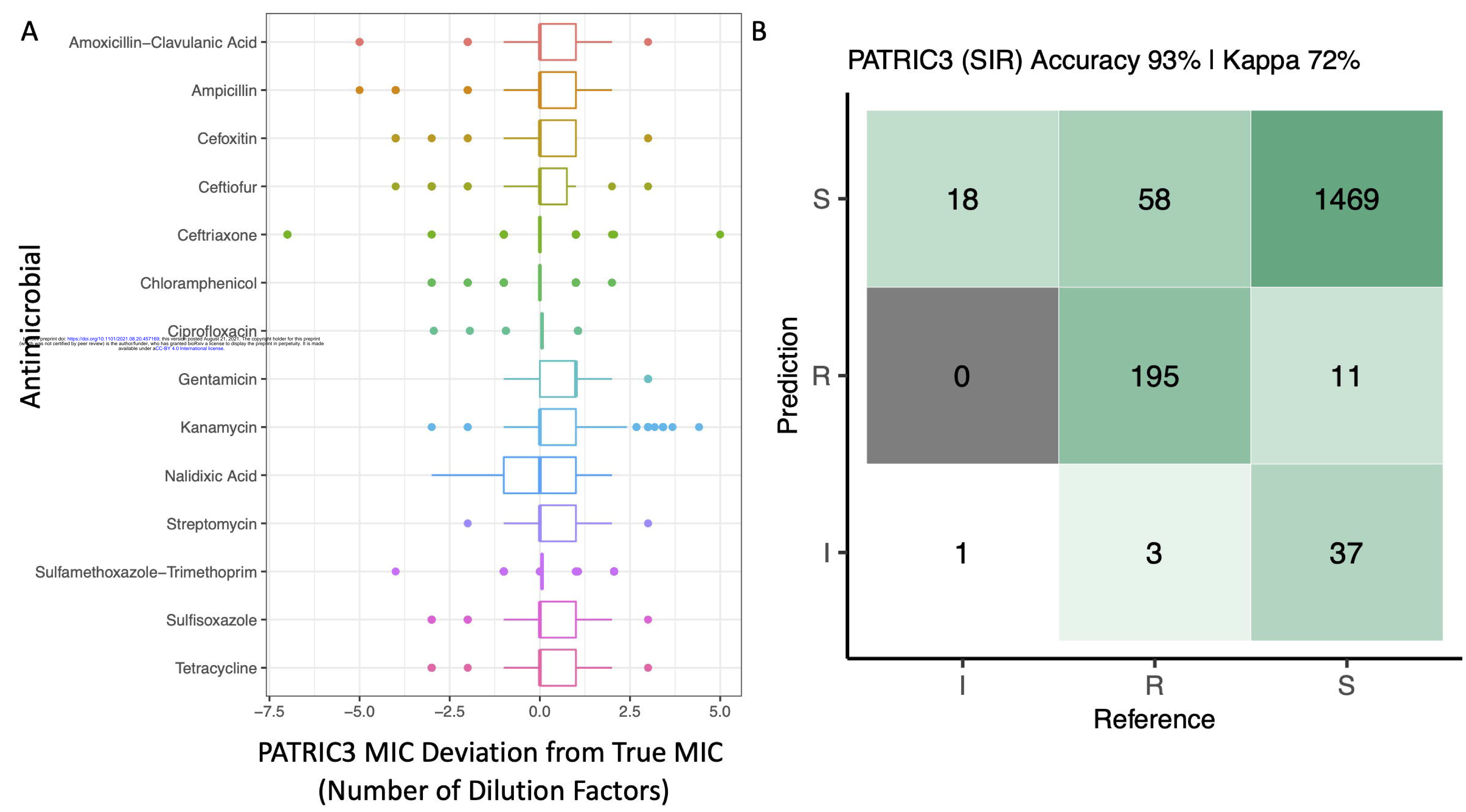




\section{A1}

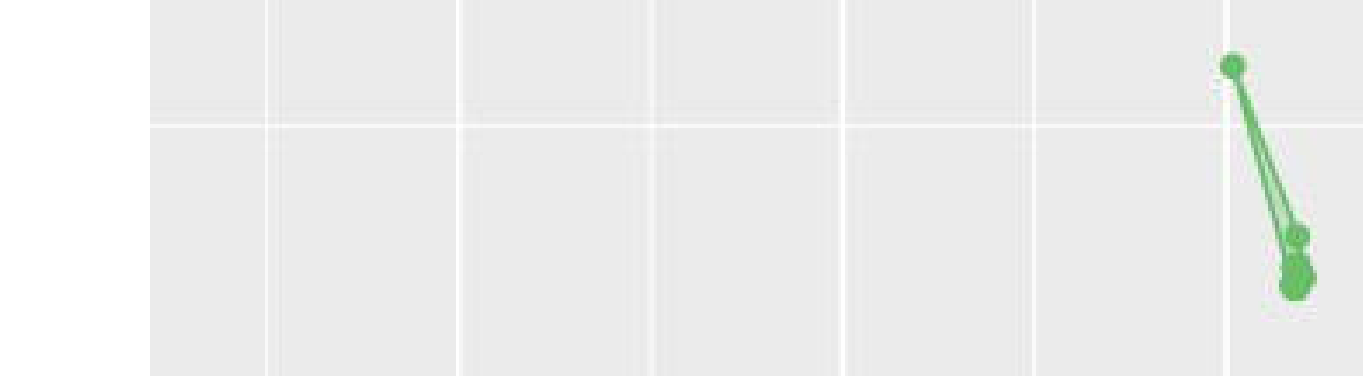

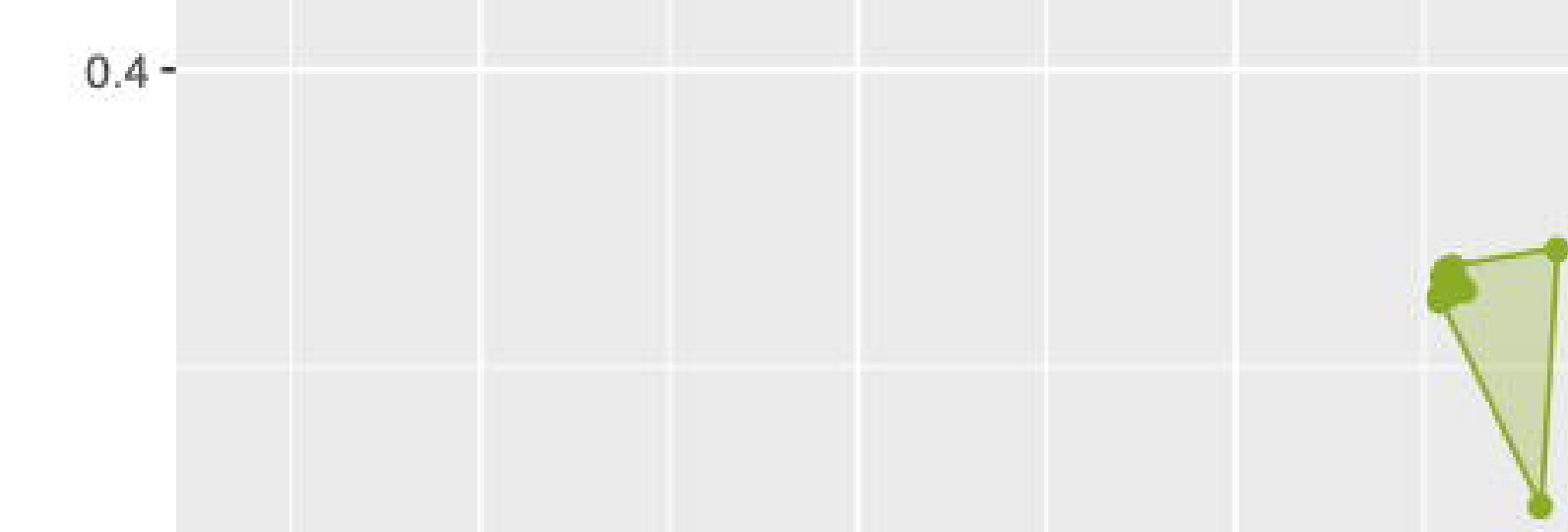

กิ้

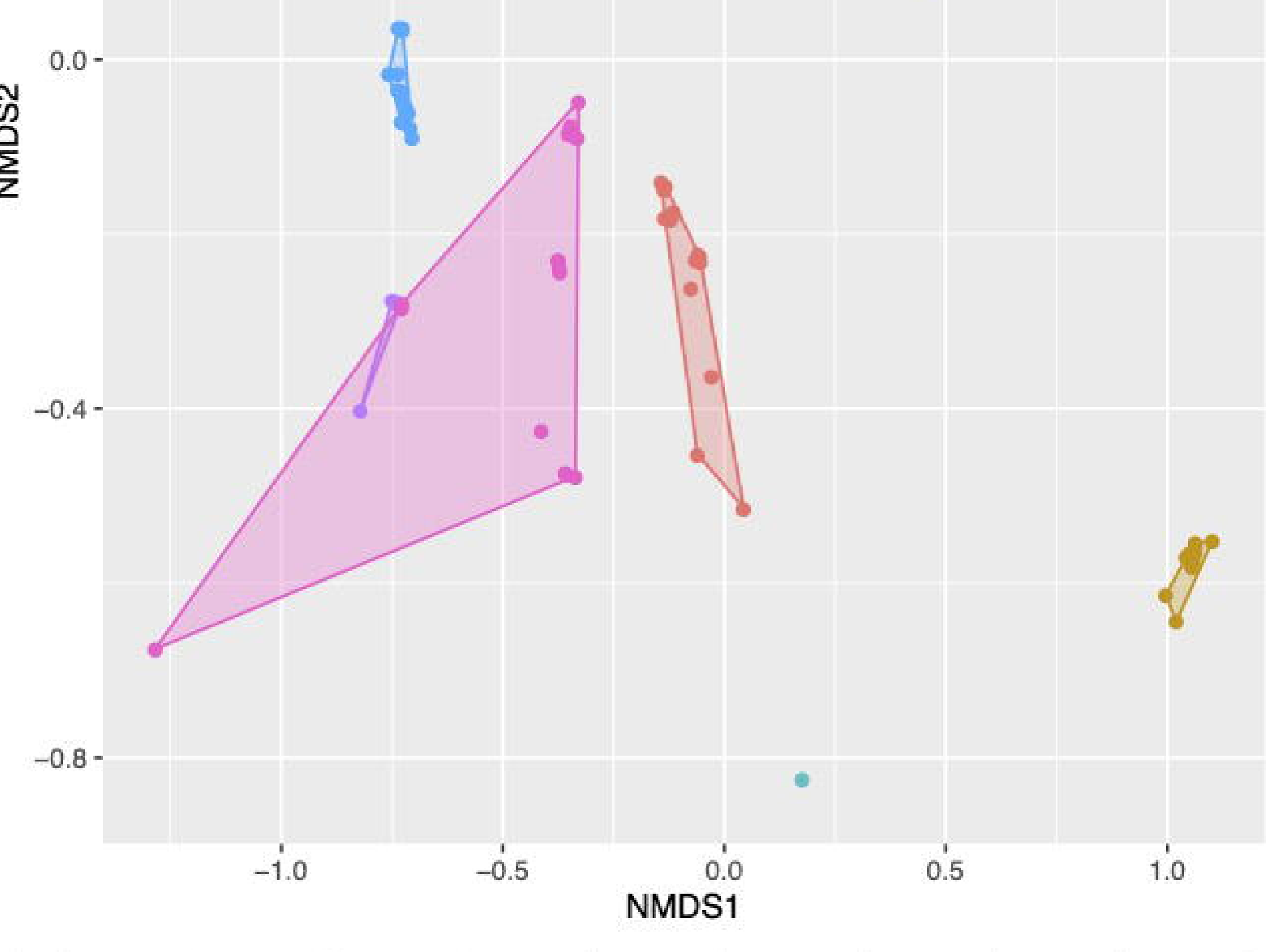

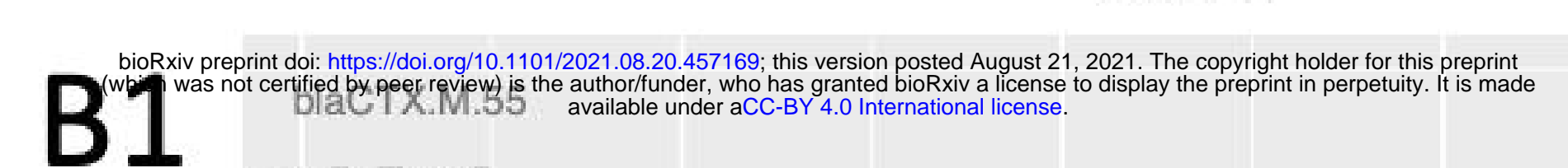

PmiB_T147P

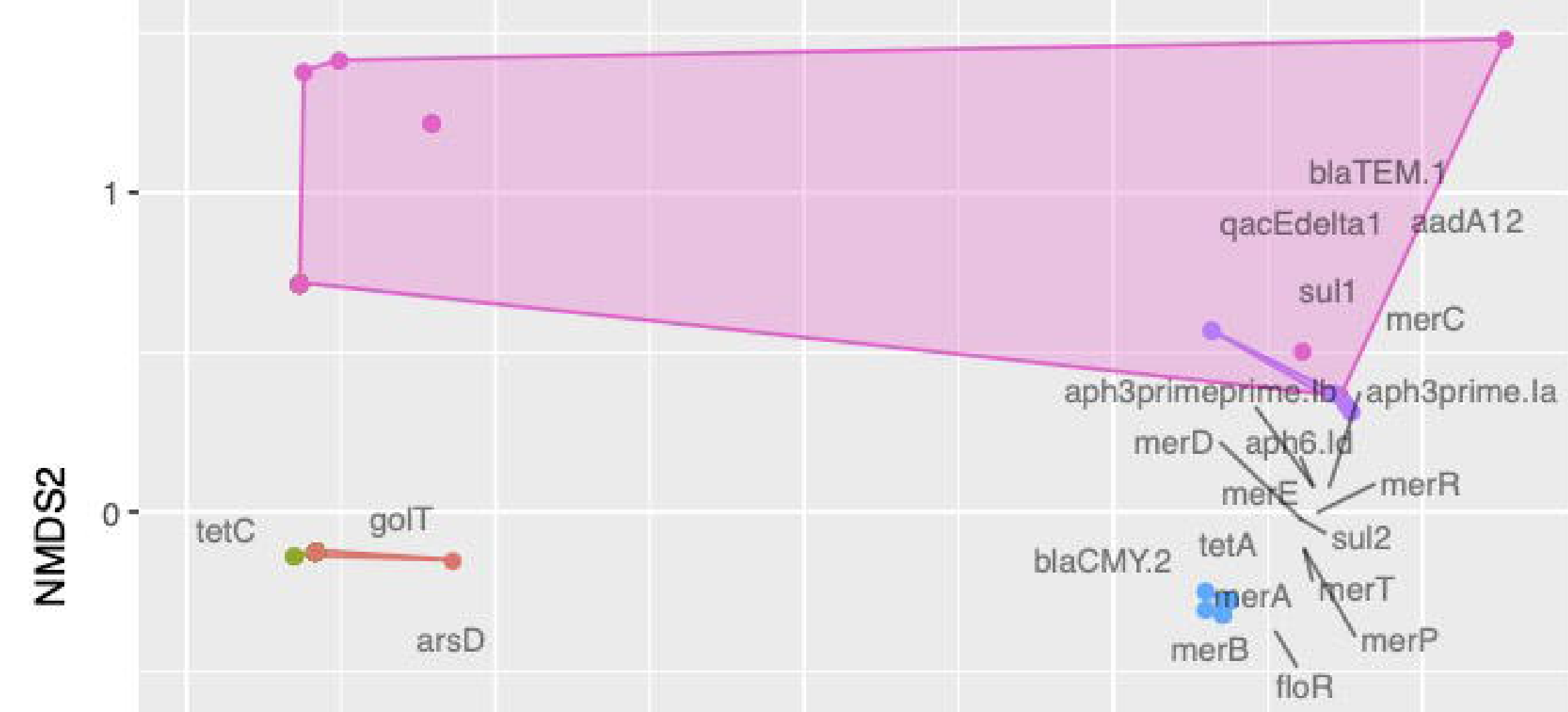

aac2primen.1Ia
I0SA7

mphA
A2

Serotype

- ANAT

CERO

MENT N

- MINN

NEWP
TCOP

$\because$ TCOP

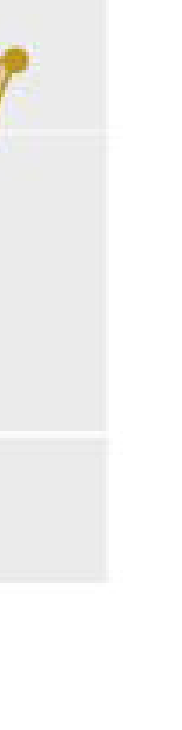

B2

blactX.M. 55

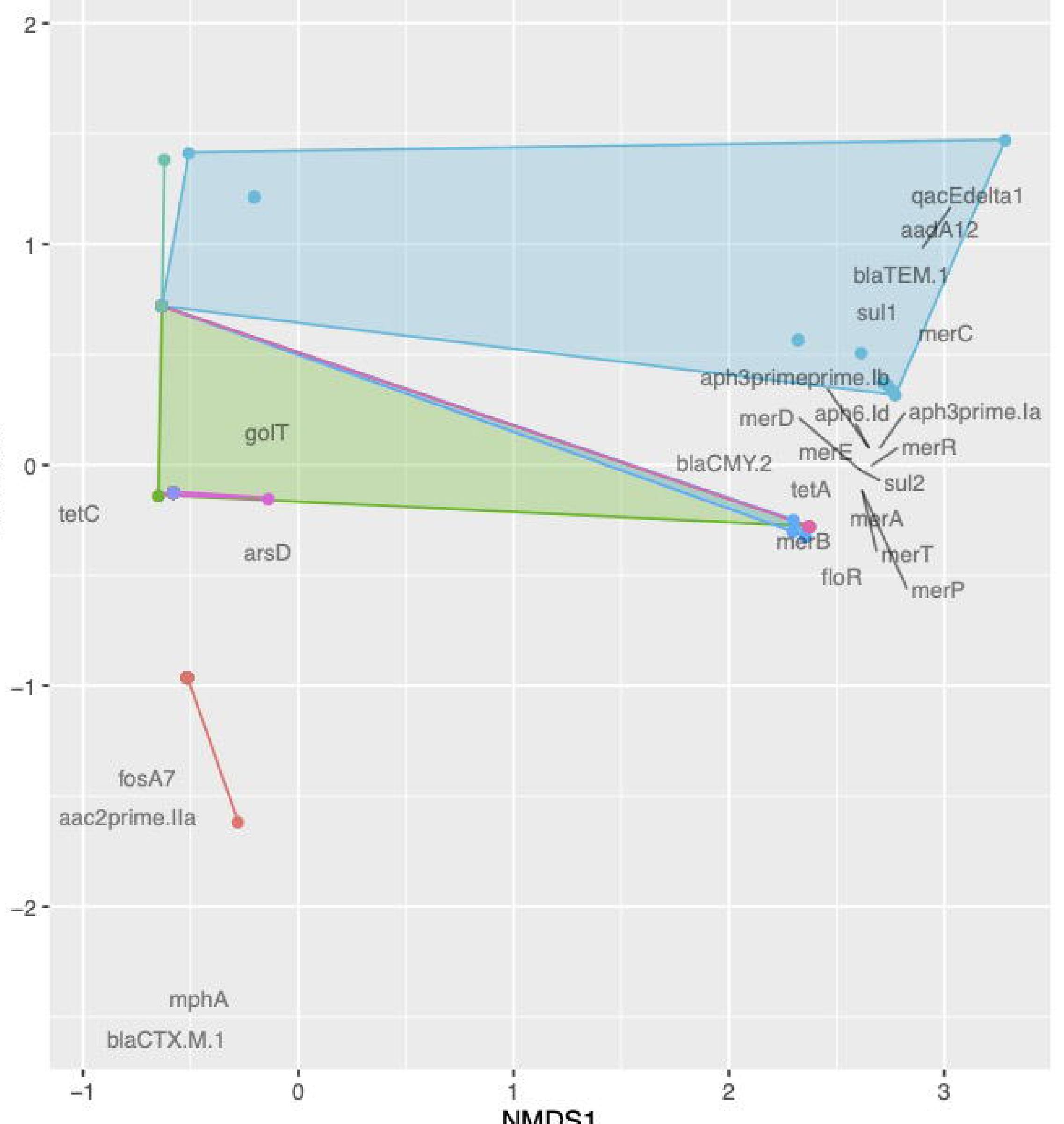

A3

Farm

$\because 1$

$\div 14$

\begin{tabular}{r|r}
$\cdot 17$ \\
\hline-19 \\
\hline$\cdot 12$
\end{tabular}

\begin{tabular}{l}
-19 \\
$-\quad 22$ \\
\hline
\end{tabular}

\begin{tabular}{l|l}
- & 25 \\
\hline- & 35 \\
\hline & 39 \\
\hline
\end{tabular}

\begin{tabular}{l}
-39 \\
\hline-42 \\
\hline-56
\end{tabular}

\begin{tabular}{l}
-56 \\
\hline-62
\end{tabular}

B3

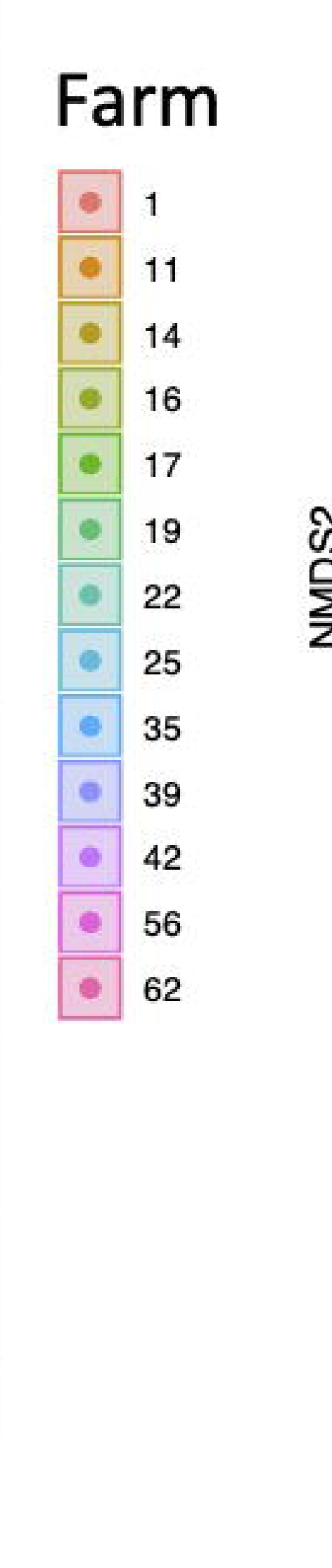

ก๊

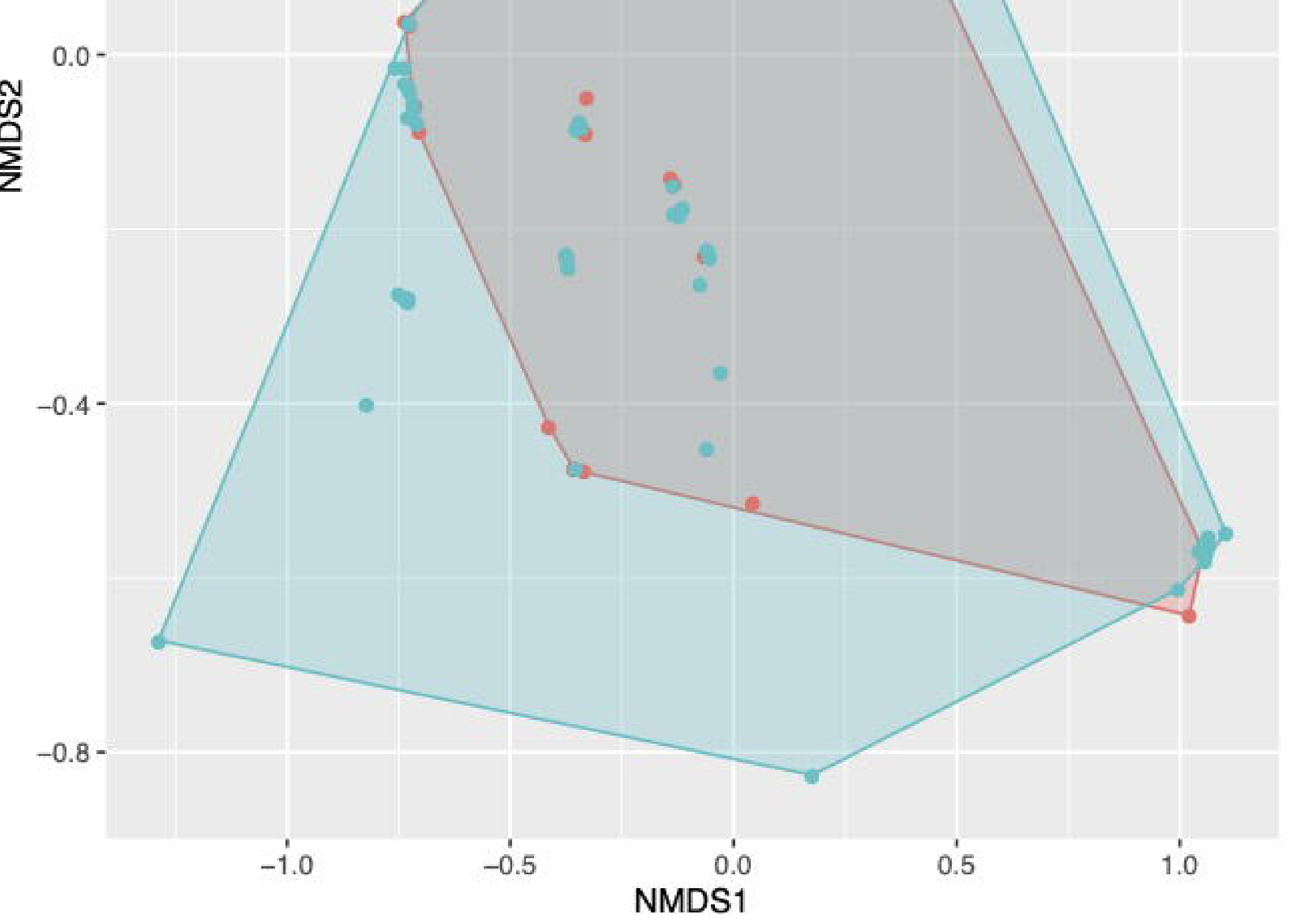

pmrB_T147P

Source

- bov
- ENV
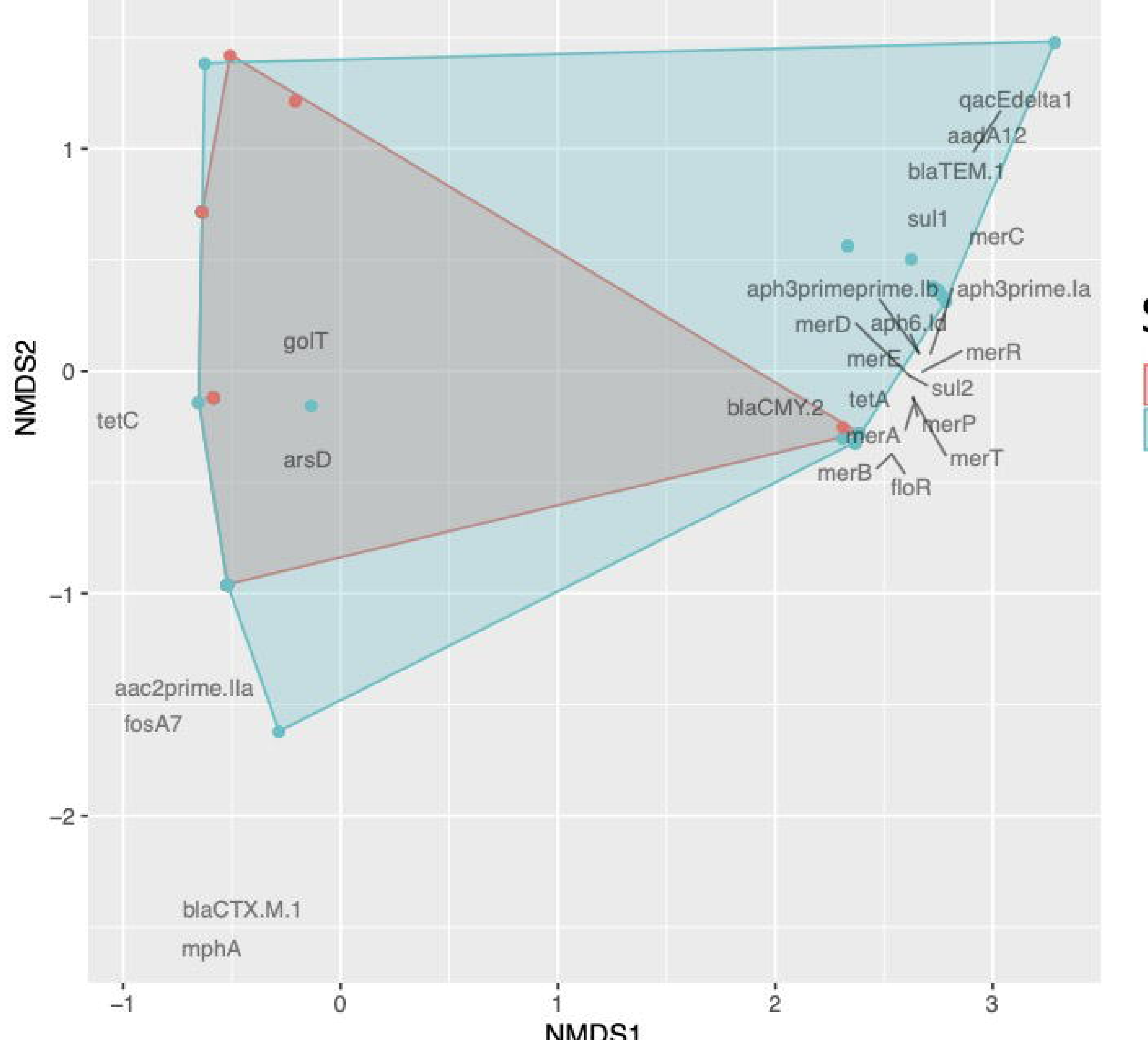

Source

- bor
-
- 


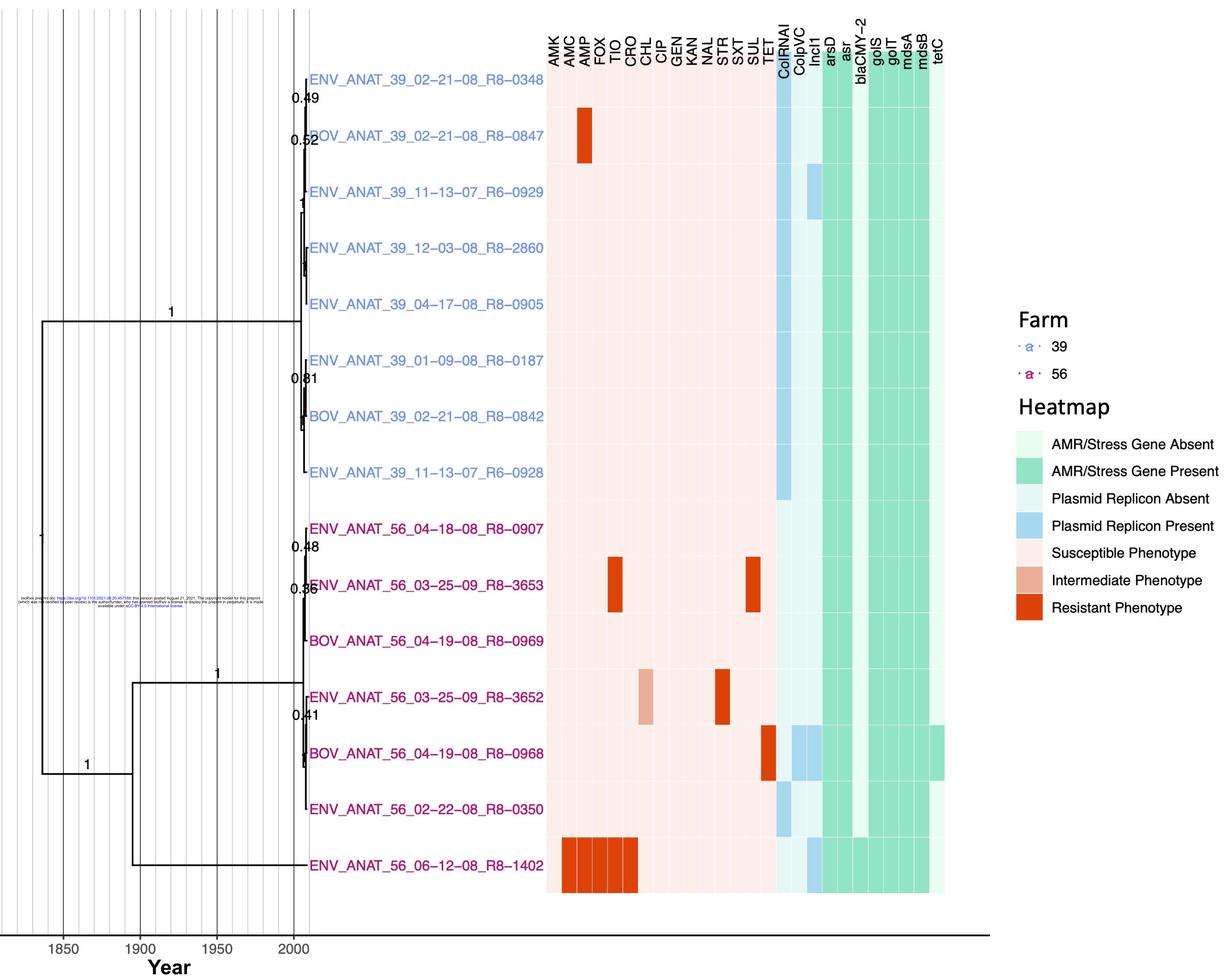




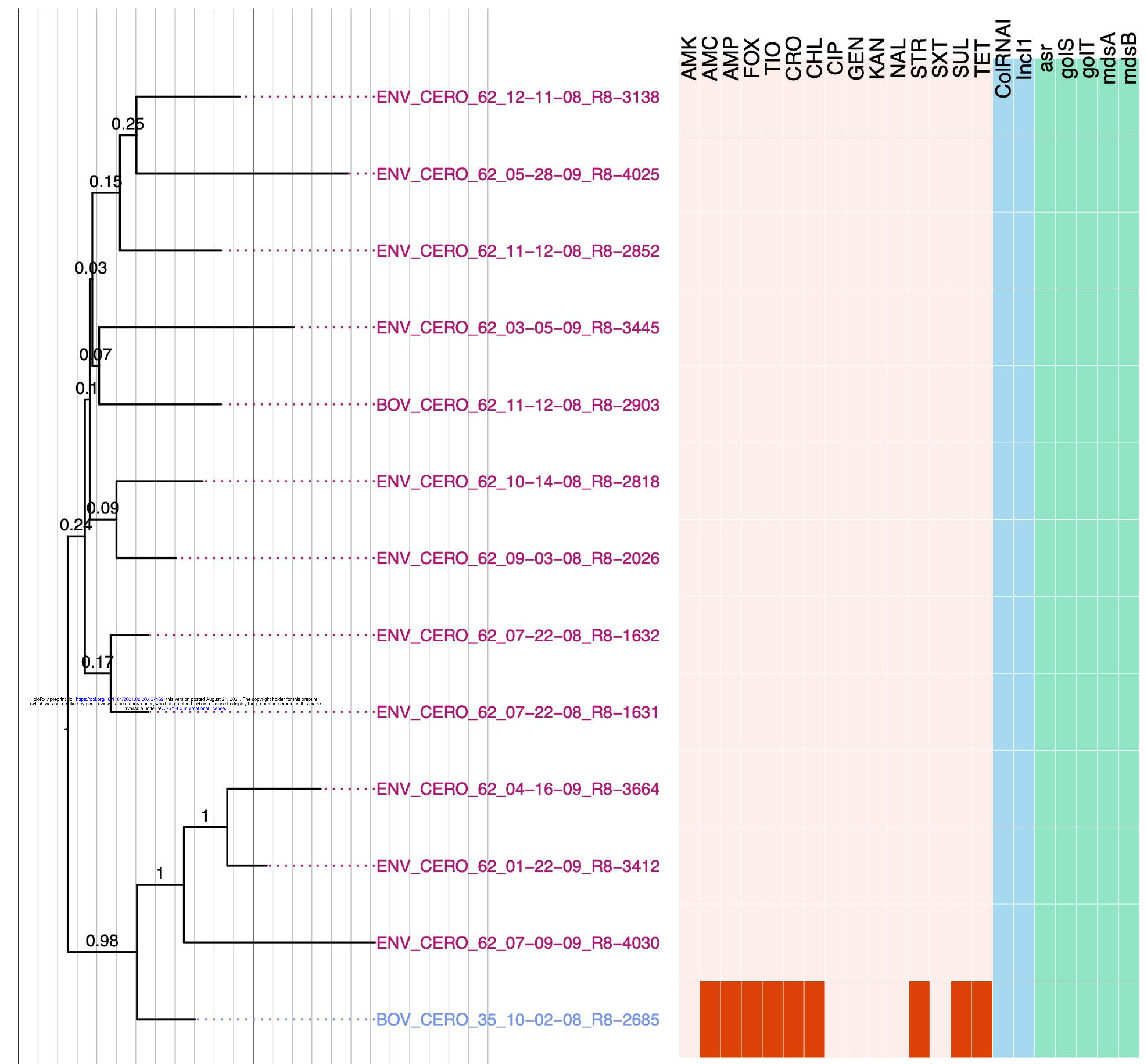

\section{Heatmap}

AMR/Stress Gene Present Plasmid Replicon Present Susceptible Phenotype

Resistant Phenotype

\section{Farm}

'a. 35

a. 62 


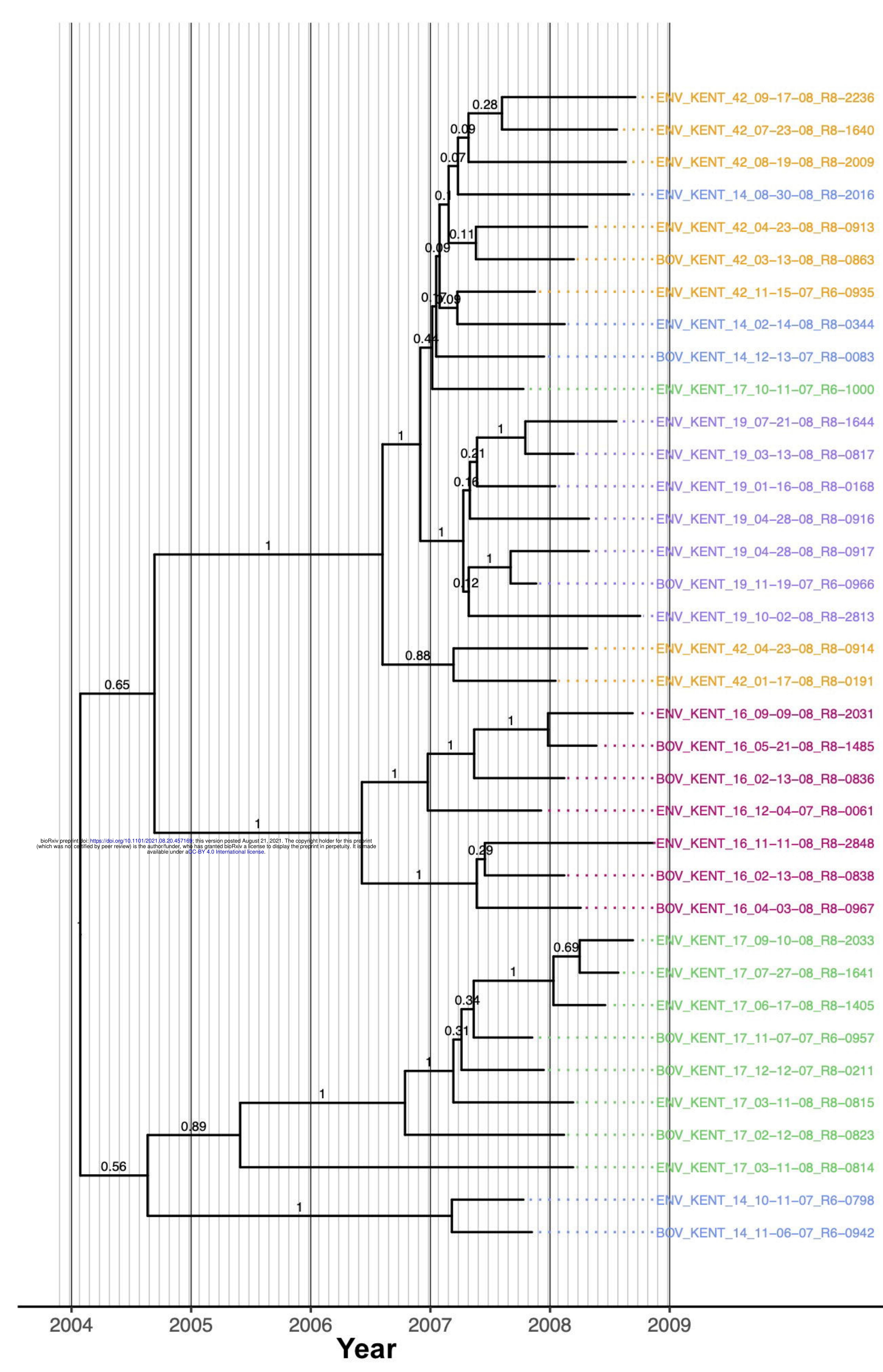

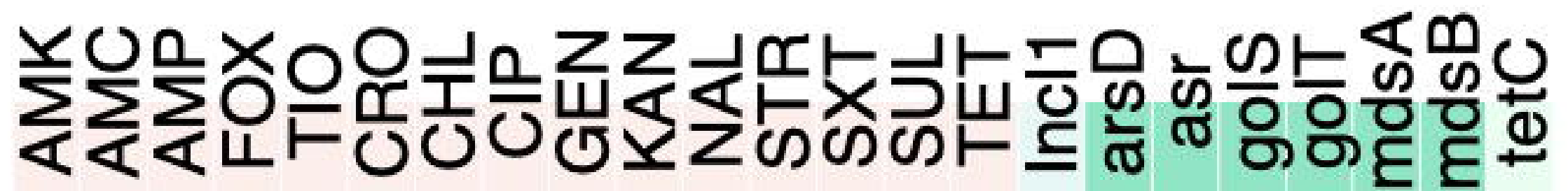

\section{Heatmap}

AMR/Stress Gene Absent AMR/Stress Gene Present Plasmid Replicon Absent Plasmid Replicon Present Susceptible Phenotype Intermediate Phenotype Resistant Phenotype

\section{Farm \\ a. 14 \\ a. 16 \\ a. 17 \\ a. . 19 \\ a. 42}




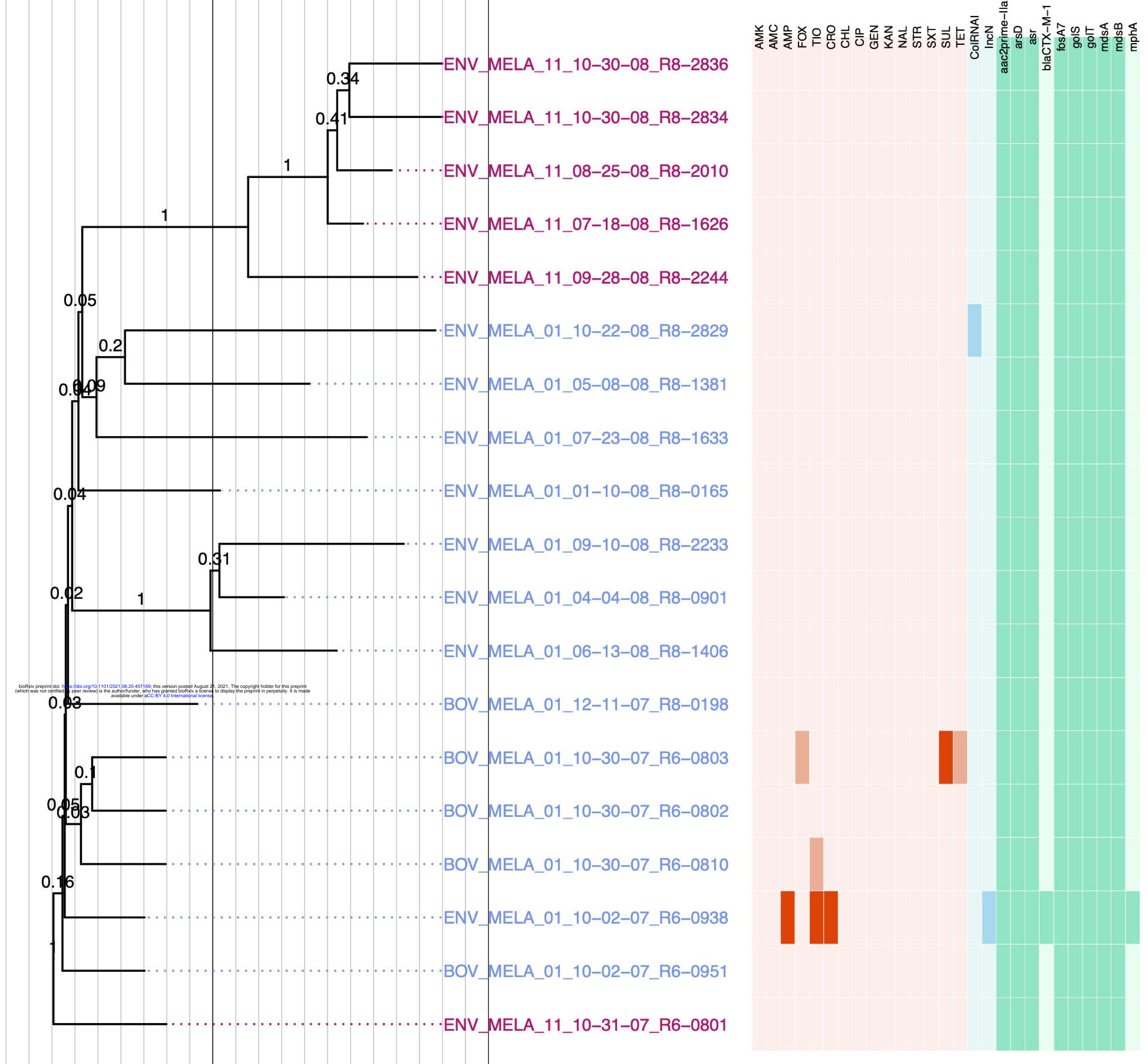

\section{Heatmap}

AMR/Stress Gene Absent AMR/Stress Gene Present Plasmid Replicon Absent

Plasmid Replicon Present Susceptible Phenotype Intermediate Phenotype Resistant Phenotype

\section{Farm}

$\cdot a \cdot 01$

$\cdot a \cdot 11$ 


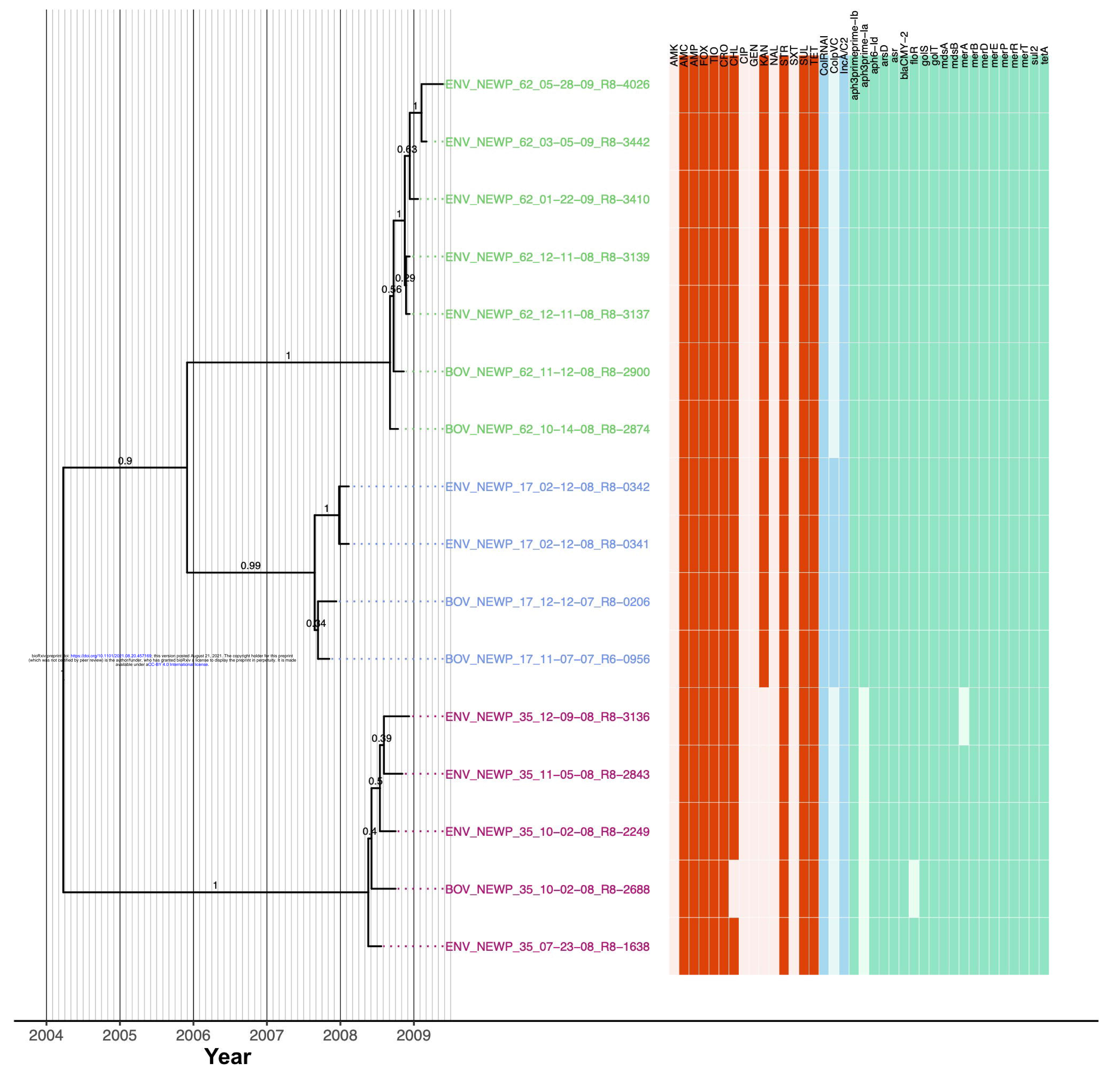

Farm

-a. 17

-a. 35

a. 62

\section{Heatmap}

AMR/Stress Gene Absent AMR/Stress Gene Present Plasmid Replicon Absent Plasmid Replicon Present Susceptible Phenotype Resistant Phenotype 
